\title{
A Cascade C-H Functionalization/Amidation Reaction for Synthesis of Azepinone Derivatives
}

\author{
Peng Bai, ${ }^{\dagger}$ Xing-Fen Huang, ${ }^{\dagger}$ Guo-Dong $\mathrm{Xu}^{\dagger}$ and Zhi-Zhen Huang ${ }^{*}, \dagger^{\dagger}$ \\ ${ }^{\dagger}$ Department of Chemistry, Zhejiang University, Hangzhou, 310028, P. R. China \\ ${ }^{\ddagger}$ State Key Laboratory of Elemento-organic Chemistry, Nankai University, Tianjin, \\ 300071, P. R. China
}

\section{Supporting Information}

\section{Contents}

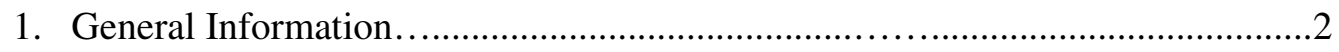

2. Optimization of the Reaction Conditions...........................................................2

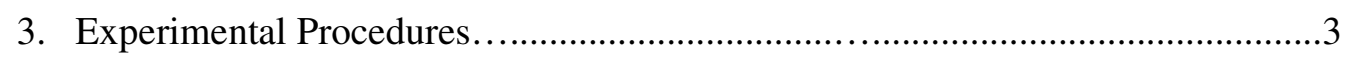

4. Characterization of Azepinone Derivatives 3 …...............................................

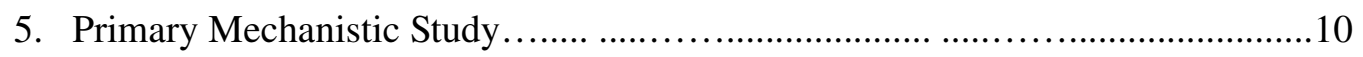

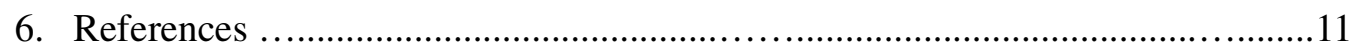

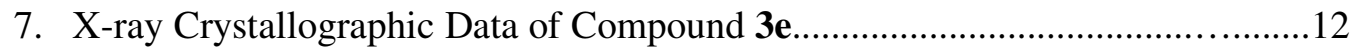

8. ${ }^{1} \mathrm{H}$ NMR, ${ }^{13} \mathrm{C}$ NMR, MS and HR-MS Spectrum of Azepinone Derivatives 3.....13

9. ${ }^{1} \mathrm{H}$ NMR Spectrum of The Mixture of Azepinone Derivatives 3a and Deuterated

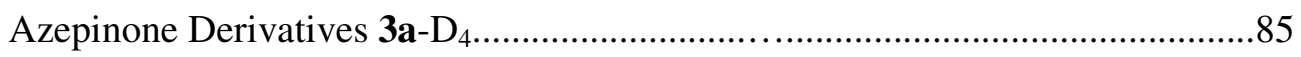




\section{General Information}

Unless otherwise indicated, all reagents were purchased from commercial distributors and used without further purification. ${ }^{1} \mathrm{H}$ and ${ }^{13} \mathrm{C}$ NMR were recorded with ${ }^{1} \mathrm{H}$ at $400 \mathrm{MHz}$ and ${ }^{13} \mathrm{C}$ at $100 \mathrm{MHz}$, respectively, using tetramethylsilane as an internal reference. Mass spectroscopy data were collected on an HRMS-EI instrument. Melting points were detected by a digital melting point apparatus and were uncorrected. Flash column chromatography was performed over silica gel 200-300. All 2-aminobiaryl 1 and diazomalonate $\mathbf{2}$ were prepared according to a reported method. ${ }^{1,2,3,4}$

\section{Optimization of the Reaction Conditions ${ }^{\text {a }}$}

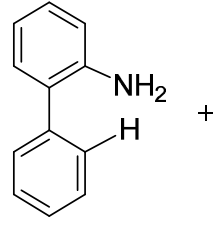

$1 \mathrm{a}$

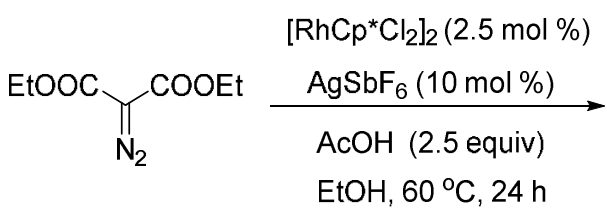

$2 a$

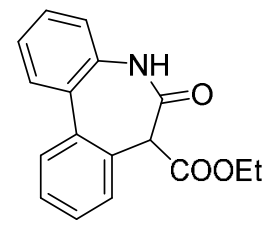

$3 a$

\begin{tabular}{|c|c|c|c|c|}
\hline entry & Catalyst system & Solvent & Additive & Yield $(\%)^{\mathbf{b}}$ \\
\hline 1 & {$\left[\mathrm{IrCp}^{*} \mathrm{Cl}_{2}\right]_{2} / \mathrm{AgSbF}_{6}$} & $\mathrm{EtOH}$ & $\mathrm{AcOH}$ & 19 \\
\hline 2 & {$\left[\mathrm{RhCp}^{*} \mathrm{Cl}_{2}\right]_{2} / \mathrm{AgSbF}_{6}$} & EtOH & AcOH & 83 \\
\hline 3 & $/ \mathrm{AgSbF}_{6}$ & $\mathrm{EtOH}$ & $\mathrm{AcOH}$ & 0 \\
\hline 4 & {$\left[\mathrm{RhCp}^{*} \mathrm{Cl}_{2}\right]_{2} / \mathrm{AgNTf}_{2}$} & $\mathrm{EtOH}$ & $\mathrm{AcOH}$ & trace \\
\hline 5 & {$\left[\mathrm{RhCp}^{*} \mathrm{Cl}_{2}\right]_{2} / \mathrm{AgOAc}$} & $\mathrm{EtOH}$ & $\mathrm{AcOH}$ & trace \\
\hline 6 & {$\left[\mathrm{RhCp}^{*} \mathrm{Cl}_{2}\right]_{2} / \quad----$} & $\mathrm{EtOH}$ & $\mathrm{AcOH}$ & trace \\
\hline 7 & {$\left[\mathrm{RhCp}^{*} \mathrm{Cl}_{2}\right]_{2} / \mathrm{AgSbF}_{6}$} & $\mathrm{EtOH}$ & $\mathrm{CsOAc}$ & 0 \\
\hline 8 & {$\left[\mathrm{RhCp}^{*} \mathrm{Cl}_{2}\right]_{2} / \mathrm{AgSbF}_{6}$} & $\mathrm{EtOH}$ & ---- & 0 \\
\hline 9 & {$\left[\mathrm{RhCp}^{*} \mathrm{Cl}_{2}\right]_{2} / \mathrm{AgSbF}_{6}$} & $\mathrm{MeOH}$ & $\mathrm{AcOH}$ & 76 \\
\hline 10 & {$\left[\mathrm{RhCp}^{*} \mathrm{Cl}_{2}\right]_{2} / \mathrm{AgSbF}_{6}$} & DCE & $\mathrm{AcOH}$ & 45 \\
\hline 11 & {$\left[\mathrm{RhCp}^{*} \mathrm{Cl}_{2}\right]_{2} / \mathrm{AgSbF}_{6}$} & $\mathrm{CH}_{3} \mathrm{CN}$ & $\mathrm{AcOH}$ & 42 \\
\hline 12 & {$\left[\mathrm{RhCp}^{*} \mathrm{Cl}_{2}\right]_{2} / \mathrm{AgSbF}_{6}$} & $\mathrm{EtOH}$ & $\mathrm{AcOH}$ & $22^{\mathrm{c}}$ \\
\hline 13 & {$\left[\mathrm{RhCp}^{*} \mathrm{Cl}_{2}\right]_{2} / \mathrm{AgSbF}_{6}$} & $\mathrm{EtOH}$ & $\mathrm{AcOH}$ & $61^{\mathrm{d}}$ \\
\hline
\end{tabular}

a) Reaction conditions: 1a $(0.10 \mathrm{mmol}), \mathbf{2 a}(0.20 \mathrm{mmol}),\left[\mathrm{RhCp}^{*} \mathrm{Cl}_{2}\right]_{2}(2.5 \mathrm{~mol} \%), \mathrm{AgSbF}_{6}$ (10 mol \%), additive (2.0 equiv), air, $60 \square$, for 24 h. b) Isolated yield. c) $\left.30{ }^{\circ} \mathrm{C} . \mathrm{d}\right) 100{ }^{\circ} \mathrm{C}$. 


\section{General Procedure for the Cascade C-H Functionalization/Amidation Reaction.}

A mixture of biphenyl-2-amine $1(0.10 \mathrm{mmol}), 2(0.20 \mathrm{mmol}),\left[\mathrm{RhCp}^{*} \mathrm{Cl}_{2}\right]_{2}(1.5$ mg, $0.0025 \mathrm{mmol}, 2.5 \mathrm{~mol} \%), \mathrm{AgSbF}_{6}(3.4 \mathrm{mg}, 0.01 \mathrm{mmol}), \mathrm{AcOH}(15 \mathrm{mg}, 0.25$ mmol, 2.5 equiv) in EtOH (2.5 mL) was stirred for $24 \mathrm{~h}$ at $60{ }^{\circ} \mathrm{C}$. After solvent was removed under reduced pressure, the residue was purified by column chromatography ( silica gel, petroleum ether / ethyl acetate $=10: 1$ to $2: 1$ as an eluent) to give desired azepinone derivative $\mathbf{3}$.

\section{Characterization of Azepinone Derivatives 3.}

Ethyl 6-oxo-6,7-dihydro-5H-dibenzo[b,d]azepine-7-carboxylate 3a

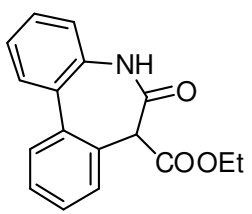

White solid (23.4 mg, $83 \%$ yield); Mp: 196-197 ${ }^{\circ} \mathrm{C} .{ }^{1} \mathrm{H}$ NMR (400 $\mathrm{MHz}, \mathrm{CDCl}_{3}$ ), $\delta$ (ppm): 9.03 (s, 1H), 7.59-7.57 (m, $\left.2 \mathrm{H}\right), 7.50-7.41$

$(\mathrm{m}, 2 \mathrm{H}), 7.37-7.32(\mathrm{~m}, 2 \mathrm{H}), 7.26-7.22(\mathrm{~m}, 1 \mathrm{H}), 7.14(\mathrm{~d}, J=8.0 \mathrm{~Hz}$, $1 \mathrm{H}), 4.73$ (s, $1 \mathrm{H}), 3.76$ (q, $J=7.1 \mathrm{~Hz}, 2 \mathrm{H}), 0.80$ (t, $J=7.0 \mathrm{~Hz}, 3 \mathrm{H}) ;{ }^{13} \mathrm{C}$ NMR $(100$ $\left.\mathrm{MHz}, \mathrm{CDCl}_{3}\right), \delta$ (ppm): 170.80, 167.16, 136.38, 134.63, 133.43, 131.69, 130.11, 130.07, 129.53, 128.96, 128.87, 128.72, 125.36, 122.06, 61.65, 60.03, 13.67; HR-MS (EI-TOF) $\left(\mathrm{M}^{+}\right)$calculated for $\mathrm{C}_{17} \mathrm{H}_{15} \mathrm{NO}_{3} 281.1052$, found 281.1054 .

Ethyl 9-methoxy-6-oxo-6,7-dihydro-5H-dibenzo[b,d]azepine-7-carboxylate $\mathbf{3 b}$

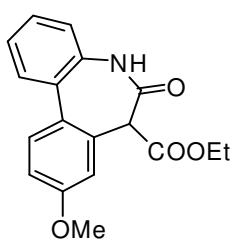

White solid (26.3 mg, $85 \%$ yield); Mp: $211-212{ }^{\circ} \mathrm{C} .{ }^{1} \mathrm{H}$ NMR (400 $\left.\mathrm{MHz}, \mathrm{CDCl}_{3}\right), \delta(\mathrm{ppm}): 8.79$ (s, $\left.1 \mathrm{H}\right), 7.54(\mathrm{dd}, J=8.0 \mathrm{~Hz}, 1.2 \mathrm{~Hz}, 1$ H), $7.49(\mathrm{~d}, J=8.4 \mathrm{~Hz}, 1 \mathrm{H}), 7.31(\mathrm{td}, J=7.6 \mathrm{~Hz}, 1.2 \mathrm{~Hz}, 1 \mathrm{H}), 7.22$ $(\mathrm{td}, J=7.5 \mathrm{~Hz}, 0.9 \mathrm{~Hz}, 1 \mathrm{H}), 7.10(\mathrm{~d}, J=7.6 \mathrm{~Hz}, 1 \mathrm{H}), 7.02(\mathrm{dd}, J=$ 8.6, 2.6 Hz, $1 \mathrm{H}), 6.90$ (d, $J=2.4 \mathrm{~Hz}, 1 \mathrm{H}), 4.66(\mathrm{~d}, J=0.8 \mathrm{~Hz}, 1 \mathrm{H}), 3.86(\mathrm{~s}, 3 \mathrm{H})$, 3.81-3.73 (m, $2 \mathrm{H}), 0.80(\mathrm{t}, J=7.2 \mathrm{~Hz}, 3 \mathrm{H}) ;{ }^{13} \mathrm{C} \mathrm{NMR}\left(100 \mathrm{MHz}, \mathrm{CDCl}_{3}\right), \delta(\mathrm{ppm})$ : 170.32 , 167.04, 160.10, 134.49, 134.18, 131.42, 130.72, 129.75, 128.88, 128.34, $125.35,121.94,115.09,114.87,61.68,60.20,55.46,13.66$; HR-MS (EI-TOF) $\left(\mathrm{M}^{+}\right)$ calculated for $\mathrm{C}_{18} \mathrm{H}_{17} \mathrm{NO}_{4} 311.1158$, found 311.1156 . 
Ethyl 9-methyl-6-oxo-6,7-dihydro-5H-dibenzo[b,d]azepine-7-carboxylate 3c

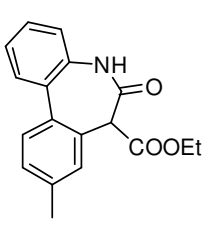

White solid (21.2 mg, $72 \%$ yield); Mp: 222-223 $\square .{ }^{1} \mathrm{H}$ NMR (400

$\left.\mathrm{MHz}, \mathrm{CDCl}_{3}\right), \delta$ (ppm): $8.90(\mathrm{~s}, 1 \mathrm{H}), 7.57$ (dd, $J=8.0 \mathrm{~Hz}, 1.2 \mathrm{~Hz}, 1$

H), $7.46(\mathrm{~d}, J=8.0 \mathrm{~Hz}, 1 \mathrm{H}), 7.34-7.27(\mathrm{~m}, 2 \mathrm{H}), 7.24-7.18(\mathrm{~m}, 2 \mathrm{H})$,

$7.12(\mathrm{~d}, J=7.6 \mathrm{~Hz}, 1 \mathrm{H}), 4.67(\mathrm{~d}, J=1.2 \mathrm{~Hz}, 1 \mathrm{H}), 3.82-3.71(\mathrm{~m}, 2 \mathrm{H})$,

$2.41(\mathrm{~s}, 3 \mathrm{H}), 0.80(\mathrm{t}, J=7.0 \mathrm{~Hz}, 3 \mathrm{H}) ;{ }^{13} \mathrm{C} \mathrm{NMR}\left(100 \mathrm{MHz}, \mathrm{CDCl}_{3}\right), \delta(\mathrm{ppm})$ : $170.71,167.26,138.81,134.47,133.50,133.22,131.67,130.78,129.90,129.72$, 129.41, 128.64, 125.31, 121.99, 61.62, 60.01, 21.00, 13.67; HR-MS (EI-TOF) $\left(\mathrm{M}^{+}\right)$ calculated for $\mathrm{C}_{18} \mathrm{H}_{17} \mathrm{NO}_{3} 295.1208$, found 295.1208.

Ethyl 9-fluoro-6-oxo-6,7-dihydro-5H-dibenzo[b,d]azepine-7-carboxylate 3d

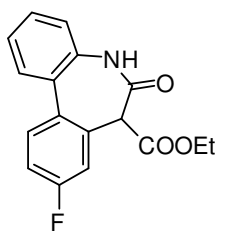

White solid (21.3 mg, $71 \%$ yield); Mp: $164-165{ }^{\circ} \mathrm{C} .{ }^{1} \mathrm{H}$ NMR $(400$

$\mathrm{MHz}, \mathrm{CDCl}_{3}$ ), $\delta$ (ppm): 8.99 (s, $\left.1 \mathrm{H}\right), 7.49-7.45$ (m, $\left.2 \mathrm{H}\right), 7.30-7.26$

(m, $1 \mathrm{H}), 7.18(\mathrm{~d}, J=7.2 \mathrm{~Hz}, 1 \mathrm{H}), 7.15-7.11(\mathrm{~m}, 1 \mathrm{H}), 7.09-7.01(\mathrm{~m}$,

$2 \mathrm{H}), 4.60$ (s, $1 \mathrm{H}), 3.69$ (q, $J=7.2 \mathrm{~Hz}, 2 \mathrm{H}), 0.80$ (t, $J=7.0 \mathrm{~Hz}, 3$

$\mathrm{H}) ;{ }^{13} \mathrm{C} \mathrm{NMR}\left(100 \mathrm{MHz}, \mathrm{CDCl}_{3}\right), \delta$ (ppm): 170.18, 166.59, $162.86(\mathrm{~d}, J=247.6 \mathrm{~Hz})$,

$135.07(\mathrm{~d}, J=8.1 \mathrm{~Hz}), 134.40,132.62(\mathrm{~d}, J=3.3 \mathrm{~Hz}), 131.26(\mathrm{~d}, J=8.4 \mathrm{~Hz}), 130.74$, 129.94, 129.04, 125.49, 122.12, $116.90(\mathrm{~d}, J=22.3 \mathrm{~Hz}), 115.96(\mathrm{~d}, J=21.2 \mathrm{~Hz})$, 61.85, 59.74, 13.64; HR-MS (EI-TOF) $\left(\mathrm{M}^{+}\right)$calculated for $\mathrm{C}_{17} \mathrm{H}_{14} \mathrm{FNO}_{3} 299.0958$, found 299.0961 .

Ethyl 9-chloro-6-oxo-6,7-dihydro-5H-dibenzo[b,d]azepine-7-carboxylate 3e

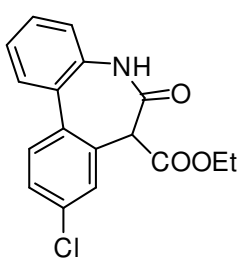

White solid (28.7 mg, $91 \%$ yield); Mp: 197-198 ${ }^{\circ} \mathrm{C} .{ }^{1} \mathrm{H}$ NMR (400 $\mathrm{MHz}, \mathrm{CDCl}_{3}$ ), $\delta$ (ppm): 9.09 (s, $\left.1 \mathrm{H}\right), 7.55-7.49$ (m, 2H), 7.45 (dd, $J$ $=8.0 \mathrm{~Hz}, 2.0 \mathrm{~Hz}, 1 \mathrm{H}), 7.39-7.35$ (m, $2 \mathrm{H}), 7.27-7.23(\mathrm{~m}, 1 \mathrm{H}), 7.14$ $(\mathrm{d}, J=8.0 \mathrm{~Hz}, 1 \mathrm{H}), 4.67(\mathrm{~s}, 1 \mathrm{H}), 3.77(\mathrm{q}, J=7.2 \mathrm{~Hz}, 2 \mathrm{H}), 0.80(\mathrm{t}$, $J=7.0 \mathrm{~Hz}, 3 \mathrm{H}) ;{ }^{13} \mathrm{C} \mathrm{NMR}\left(100 \mathrm{MHz}, \mathrm{CDCl}_{3}\right), \delta(\mathrm{ppm}): 170.21,166.56,134.96$, $134.68,134.53,130.76,130.62,129.95,129.89,129.32,129.02,125.54,122.17$, 61.87, 59.60, 13.65; HR-MS (EI-TOF) $\left(\mathrm{M}^{+}\right)$calculated for $\mathrm{C}_{17} \mathrm{H}_{14} \mathrm{ClNO}_{3} 315.0662$, 
found 315.0663 .

Diethyl 6-oxo-6,7-dihydro-5H-dibenzo[b,d]azepine-7,9-dicarboxylate $3 f$

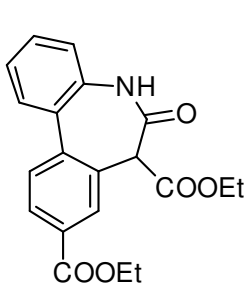

White soild (21.2 mg, $60 \%$ yield); Mp: 231-232 ${ }^{\circ} \mathrm{C}{ }^{1} \mathrm{H}$ NMR (400

MHz, d ${ }_{6}$-DMSO), $\delta$ (ppm): 10.33 (s, 1H), 8.12-8.05(m, 2H), 7.77 (d, $J=8.0 \mathrm{~Hz}, 1 \mathrm{H}), 7.62(\mathrm{dd}, J=8.0 \mathrm{~Hz}, 1.2 \mathrm{~Hz}, 1 \mathrm{H}), 7.46-7.42(\mathrm{~m}$, 1H), 7.29-7.25 (m, 1H), 7.18 (d, $J=7.6 \mathrm{~Hz}, 1 \mathrm{H}), 5.39$ (d, $J=1.2 \mathrm{~Hz}$, $1 \mathrm{H}), 4.37$ (q, $J=7.2 \mathrm{~Hz}, 2 \mathrm{H}), 3.66-3.59(\mathrm{~m}, 2 \mathrm{H}), 1.36$ (t, $J=7.0 \mathrm{~Hz}, 3 \mathrm{H}), 0.67$ (t, $J=$ $7.0 \mathrm{~Hz}, 3 \mathrm{H}) ;{ }^{13} \mathrm{C}$ NMR (100 MHz, DMSO-d 6 ), $\delta$ (ppm): 169.26, 167.40, 165.77, $141.37,136.07,134.69,131.07,130.49,130.43,130.18,130.03,129.52,125.15$, 122.67, 61.43, 61.34, 58.98, 14.66, 13.91; HR-MS (EI-TOF) $\left(\mathrm{M}^{+}\right)$calculated for $\mathrm{C}_{20} \mathrm{H}_{19} \mathrm{NO}_{5}$ 353.1263, found 353.1267.

Ethyl 9-nitro-6-oxo-6,7-dihydro-5H-dibenzo[b,d]azepine-7-carboxylate 3g

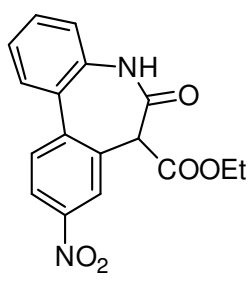

Yellow soild (17.2 mg, $53 \%$ yield); Mp: $241-242{ }^{\circ} \mathrm{C} ;{ }^{1} \mathrm{H}$ NMR (400 MHz, d ${ }_{6}$-DMSO), $\delta$ (ppm): 10.45 (s, $\left.1 \mathrm{H}\right), 8.51$ (d, $\left.J=2.4 \mathrm{~Hz}, 1 \mathrm{H}\right)$, $8.34(\mathrm{dd}, J=8.8 \mathrm{~Hz}, 2.4 \mathrm{~Hz}, 1 \mathrm{H}), 7.91(\mathrm{~d}, J=8.4 \mathrm{~Hz}, 1 \mathrm{H})$, 7.67-7.65 (m, 1H), 7.51-7.47 (m, 1H), 7.32-7.28 (m, 1H), 7.21 (d, $J$ $=7.2 \mathrm{~Hz}, 1 \mathrm{H}), 5.39(\mathrm{~d}, J=0.8 \mathrm{~Hz}, 1 \mathrm{H}), 3.73-3.63(\mathrm{~m}, 2 \mathrm{H}), 0.68(\mathrm{t}, J=7.2 \mathrm{~Hz}, 3 \mathrm{H})$; ${ }^{13} \mathrm{C}$ NMR (100 MHz, DMSO-d 6 ), $\delta$ (ppm): 168.83, 167.01, 147.53, 143.42, 136.17, $135.47,131.17,130.71,130.68,129.66,125.34,125.16,123.80,122.82,61.59,58.47$, 13.90; HR-MS (EI-TOF) $\left(\mathrm{M}^{+}\right)$calculated for $\mathrm{C}_{17} \mathrm{H}_{14} \mathrm{~N}_{2} \mathrm{O}_{5} 326.0903$, found 326.0911 .

\section{Ethyl 3-methyl-6-oxo-6,7-dihydro-5H-dibenzo[b,d]azepine-7-carboxylate 3h}

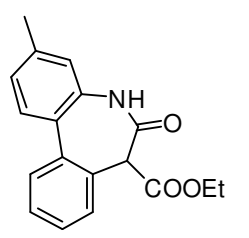

White solid (22.3 mg, $76 \%$ yield); Mp: 184-185 ${ }^{\circ} \mathrm{C} .{ }^{1} \mathrm{H}$ NMR (400 $\left.\mathrm{MHz}, \mathrm{CDCl}_{3}\right), \delta(\mathrm{ppm}): 8.57(\mathrm{~s}, 1 \mathrm{H}), 7.55(\mathrm{~d}, J=7.6 \mathrm{~Hz}, 1 \mathrm{H})$, 7.48-7.39 (m, $3 \mathrm{H}), 7.35(\mathrm{~d}, J=7.2 \mathrm{~Hz}, 1 \mathrm{H}), 7.06(\mathrm{~d}, J=8.0 \mathrm{~Hz}, 1$ H), $6.92(\mathrm{~s}, 1 \mathrm{H}), 4.71(\mathrm{~d}, J=0.8 \mathrm{~Hz}, 1 \mathrm{H}), 3.84-3.71(\mathrm{~m}, 2 \mathrm{H}), 2.37$ $(\mathrm{s}, 3 \mathrm{H}), 0.80(\mathrm{t}, J=7.2 \mathrm{~Hz}, 3 \mathrm{H}) ;{ }^{13} \mathrm{C} \mathrm{NMR}\left(100 \mathrm{MHz}, \mathrm{CDCl}_{3}\right), \delta$ (ppm): 170.54, $167.25,139.22$, 136.38, 134.39, 133.18, 130.09, 129.94, 129.29, 128.90, 128.82, $128.44,126.42,122.18,61.57,60.06,20.99,13.63 ;$ HR-MS (EI-TOF) $\left(\mathrm{M}^{+}\right)$ 
calculated for $\mathrm{C}_{18} \mathrm{H}_{17} \mathrm{NO}_{3} 295.1208$, found 295.1210.

Ethyl 2-methyl-6-oxo-6, 7-dihydro-5H-dibenzo[b,d]azepine-7-carboxylate 3i

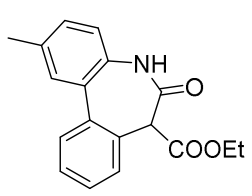

White solid (19.9 mg, $67 \%$ yield); Mp: 205-206 ${ }^{\circ} \mathrm{C} ;{ }^{1} \mathrm{H}$ NMR (400

$\left.\mathrm{MHz}, \mathrm{CDCl}_{3}\right), \delta(\mathrm{ppm}): 8.73(\mathrm{~s}, 1 \mathrm{H}), 7.58(\mathrm{dd}, J=7.6 \mathrm{~Hz}, 1.6 \mathrm{~Hz}, 1$

H), 7.49-7.34 (m, 4 H), 7.14 (dd, $J=8.2 \mathrm{~Hz}, 1.4 \mathrm{~Hz}, 1 \mathrm{H}), 7.00$ (d, $J$ $=8.0 \mathrm{~Hz}, 1 \mathrm{H}), 4.70(\mathrm{~s}, 1 \mathrm{H}), 3.82-3.73(\mathrm{~m}, 2 \mathrm{H}), 2.38(\mathrm{~s}, 3 \mathrm{H}), 0.82(\mathrm{t}, J=8.0 \mathrm{~Hz}, 3$ $\mathrm{H}) ;{ }^{13} \mathrm{C} \mathrm{NMR}\left(100 \mathrm{MHz}, \mathrm{CDCl}_{3}\right), \delta$ (ppm): 170.59, 167.25, 136.46, 135.01, 133.39, $132.16,131.56,130.34,130.11,129.75,129.41,128.80,128.62,121.98,61.59,60.03$, 20.94, 13.66; HR-MS (EI-TOF) $\left(\mathrm{M}^{+}\right)$calculated for $\mathrm{C}_{18} \mathrm{H}_{17} \mathrm{NO}_{3} 295.1208$, found 295.1210.

Ethyl 9-chloro-3-methyl-6-oxo-6,7-dihydro-5H-dibenzo[b,d]azepine-7-carboxylate 3j

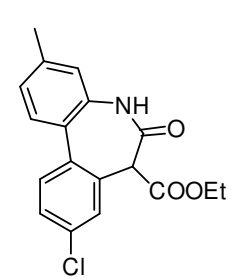

White solid (24.2 mg, $74 \%$ yield); Mp: 207-208 ${ }^{\circ} \mathrm{C} .{ }^{1} \mathrm{H}$ NMR (400 MHz, d d $\left.^{-D M S O}\right), \delta(\mathrm{ppm}): 10.24(\mathrm{~s}, 1 \mathrm{H}), 7.64(\mathrm{~d}, J=2.0 \mathrm{~Hz}, 1 \mathrm{H})$, 7.60-7.52 (m, $2 \mathrm{H}), 7.44(\mathrm{~d}, J=8.0 \mathrm{~Hz}, 1 \mathrm{H}), 7.06(\mathrm{~d}, J=8.0 \mathrm{~Hz}, 1 \mathrm{H})$, $6.95(\mathrm{~s}, 1 \mathrm{H}), 5.00(\mathrm{~s}, 1 \mathrm{H}), 3.68(\mathrm{q}, J=6.9 \mathrm{~Hz}, 2 \mathrm{H}), 2.31(\mathrm{~s}, 3 \mathrm{H})$, 0.67 (t, $J=7.0 \mathrm{~Hz}, 3 \mathrm{H}) ;{ }^{13} \mathrm{C}$ NMR (100 MHz, $\left.\mathrm{d}_{6}-\mathrm{DMSO}\right), \delta$ (ppm): 173.76, 172.05, $143.94,140.57,140.51,140.31,137.89,135.89,134.80,134.60,133.59,132.34$, 130.80, 127.42, 66.10, 63.49, 25.81, 18.61; HR-MS (EI-TOF) $\left(\mathrm{M}^{+}\right)$calculated for $\mathrm{C}_{18} \mathrm{H}_{16} \mathrm{ClNO}_{3} 329.0819$, found 329.0822 .

\section{Ethyl 3-fluoro-9-methoxy-6-oxo-6,7-dihydro-5H-dibenzo[b,d]azepine-7-carboxylate}

\section{$3 \mathbf{k}$}

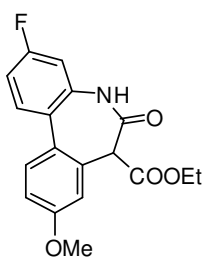

White solid (19 mg, $58 \%$ yield); Mp: $218-219{ }^{\circ} \mathrm{C} .{ }^{1} \mathrm{H}$ NMR $(400 \mathrm{MHz}$, $\left.\mathrm{CDCl}_{3}\right), \delta(\mathrm{ppm}): 8.90(\mathrm{~s}, 1 \mathrm{H}), 7.45-7.42(\mathrm{~m}, 1 \mathrm{H}), 7.36(\mathrm{~d}, J=8.4 \mathrm{~Hz}$, $1 \mathrm{H}), 6.94(\mathrm{dd}, J=8.4 \mathrm{~Hz}, 2.4 \mathrm{~Hz}, 1 \mathrm{H}), 6.88(\mathrm{td}, J=8.4 \mathrm{~Hz}, 2.3 \mathrm{~Hz}, 1$ H), $6.82(\mathrm{~d}, J=2.4 \mathrm{~Hz}, 1 \mathrm{H}), 6.78(\mathrm{dd}, J=9.2 \mathrm{~Hz}, 2.4 \mathrm{~Hz}, 1 \mathrm{H}), 4.59(\mathrm{~s}$, $1 \mathrm{H}), 3.79$ (s, $3 \mathrm{H}), 3.76-3.73$ (m, $2 \mathrm{H}), 0.79$ (t, $J=7.0 \mathrm{~Hz}, 3 \mathrm{H}) ;{ }^{13} \mathrm{C}$ NMR $(100 \mathrm{MHz}$, $\left.\mathrm{CDCl}_{3}\right), \delta(\mathrm{ppm}): 170.20,166.90,162.22(\mathrm{~d}, J=246.8 \mathrm{~Hz}), 160.14,135.45(\mathrm{~d}, J=$ 
$10.1 \mathrm{~Hz}), 134.06,131.34(\mathrm{~d}, J=8.8 \mathrm{~Hz}), 130.61,128.08,127.66,115.08(\mathrm{~d}, J=29.7$ $\mathrm{Hz}), 112.74(\mathrm{~d}, J=21.4 \mathrm{~Hz}), 108.51(\mathrm{~d}, J=23.7 \mathrm{~Hz}), 61.82,60.16,55.49,13.71$; HR-MS (EI-TOF) $\left(\mathrm{M}^{+}\right.$) calculated for $\mathrm{C}_{18} \mathrm{H}_{16} \mathrm{FNO}_{4} 329.1063$, found 329.1062 .

\section{Ethyl 9-methoxy-3-methyl-6-oxo-6,7-dihydro-5H-dibenzo[b,d]azepine-7-carboxylate}

31

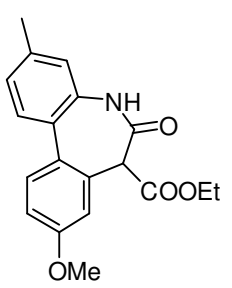

White solid (20.5 mg, $63 \%$ yield); Mp: $234-235{ }^{\circ} \mathrm{C} .{ }^{1} \mathrm{H}$ NMR (400 MHz, $\mathrm{d}_{6}$-DMSO), $\delta$ (ppm): $10.12(\mathrm{~s}, 1 \mathrm{H}), 7.48(\mathrm{~d}, J=8.8 \mathrm{~Hz}, 1 \mathrm{H})$, $7.39(\mathrm{~d}, J=8.0 \mathrm{~Hz}, 1 \mathrm{H}), 7.10(\mathrm{~d}, J=2.4 \mathrm{~Hz}, 1 \mathrm{H}), 7.07-7.01(\mathrm{~m}, 2$ H), $6.92(\mathrm{~s}, 1 \mathrm{H}), 4.84(\mathrm{~s}, 1 \mathrm{H}), 3.82(\mathrm{~s}, 3 \mathrm{H}), 3.71-3.62(\mathrm{~m}, 2 \mathrm{H})$, $2.30(\mathrm{~s}, 3 \mathrm{H}), 0.67$ (t, $J=7.2 \mathrm{~Hz}, 3 \mathrm{H}) ;{ }^{13} \mathrm{C} \mathrm{NMR}\left(100 \mathrm{MHz}, \mathrm{d}_{6}\right.$-DMSO), $\delta$ (ppm): $169.20,167.67,159.72,137.90,135.30,135.20,130.53,129.71,129.28,128.46$, 125.80, 122.50, 115.39, 114.80, 61.13, 59.58, 55.82, 21.01, 13.87; HR-MS (EI-TOF) $\left(\mathrm{M}^{+}\right)$calculated for $\mathrm{C}_{19} \mathrm{H}_{19} \mathrm{NO}_{4} 325.1314$, found 325.1313 .

Ethyl 9-chloro-2-methyl-6-oxo-6,7-dihydro-5H-dibenzo[b,d]azepine-7-carboxylate $3 m$

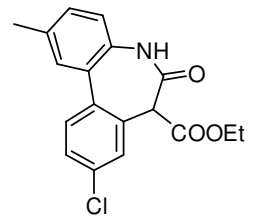

Light yellow solid (24 mg, $73 \%$ yield). Mp: $225-226{ }^{\circ} \mathrm{C} ;{ }^{1} \mathrm{H}$ NMR (400 MHz, $\left.\mathrm{CDCl}_{3}\right), \delta(\mathrm{ppm}): 8.82(\mathrm{~s}, 1 \mathrm{H}), 7.51(\mathrm{~d}, J=8.0 \mathrm{~Hz}, 1 \mathrm{H})$, $7.44(\mathrm{dd}, J=8.4 \mathrm{~Hz}, 2.0 \mathrm{~Hz}, 1 \mathrm{H}), 7.36-7.33(\mathrm{~m}, 1 \mathrm{H}), 7.17$ (dd, $J=$ $7.8 \mathrm{~Hz}, 1.4 \mathrm{~Hz}, 1 \mathrm{H}), 7.01(\mathrm{~d}, J=8.0 \mathrm{~Hz}, 1 \mathrm{H}), \quad 4.64(\mathrm{~s}, 1 \mathrm{H}), \quad 3.82-3.74(\mathrm{~m}, 2 \mathrm{H})$, 2.38 (s, $3 \mathrm{H}), 0.82$ (t, $J=7.0 \mathrm{~Hz}, 3 \mathrm{H}) ;{ }^{13} \mathrm{C} \mathrm{NMR}\left(100 \mathrm{MHz}, \mathrm{CDCl}_{3}\right), \delta$ (ppm): 169.99, $166.65,135.26,135.05,134.65,134.54,132.07,130.65,130.49,130.12,129.94$, 128.93, 122.11, 61.81, 59.61, 20.93, 13.65; HR-MS (EI-TOF) $\left(\mathrm{M}^{+}\right)$calculated for $\mathrm{C}_{18} \mathrm{H}_{16} \mathrm{ClNO}_{3} 329.0819$, found 329.0823 .

Ethyl 6-oxo-6,7-dihydro-5H-benzo[b]naphtho[2,3-d]azepine-7-carboxylate 3n

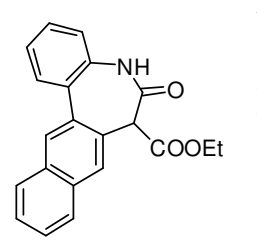

White solid (23.1 mg, $70 \%$ yield); Mp: 246-247 ${ }^{\circ} \mathrm{C} .{ }^{1} \mathrm{H}$ NMR (400 MHz, $\mathrm{d}_{6}$-DMSO), $\delta$ (ppm): 10.23 (s, $\left.1 \mathrm{H}\right), 8.17$ (s, $\left.1 \mathrm{H}\right), 8.07-8.04$ 
(m, $2 \mathrm{H}), 7.99-7.97(\mathrm{~m}, 1 \mathrm{H}), 7.71(\mathrm{~d}, J=7.6 \mathrm{~Hz}, 1 \mathrm{H}), 7.60-7.58(\mathrm{~m}, 2 \mathrm{H}), 7.42(\mathrm{t}, J=$ $7.4 \mathrm{~Hz}, 1 \mathrm{H}), 7.29$ (t, $J=7.4 \mathrm{~Hz}, 1 \mathrm{H}), 7.19$ (d, $J=8.0 \mathrm{~Hz}, 1 \mathrm{H}), 5.08$ (s, $1 \mathrm{H})$, 3.73-3.61 (m, $2 \mathrm{H}), 0.70(\mathrm{t}, J=7.0 \mathrm{~Hz}, 3 \mathrm{H}) ;{ }^{13} \mathrm{C}$ NMR (100 MHz, $\mathrm{d}_{6}$-DMSO), $\delta$ (ppm): 169.27, 167.83, 136.05, 135.13, 133.14, 132.81, 132.79, 131.77, 130.75, $129.36,128.90,128.54,127.68,127.25,127.15,125.24,122.71,61.32,59.87,13.94$; HR-MS (EI-TOF) $\left(\mathrm{M}^{+}\right)$calculated for $\mathrm{C}_{21} \mathrm{H}_{17} \mathrm{NO}_{3} 331.1208$, found 331.1208.

Ethyl 2-methyl-6-oxo-6,7-dihydro-5H-benzo[b]naphtho[2,3-d]azepine-7-carboxylate 30

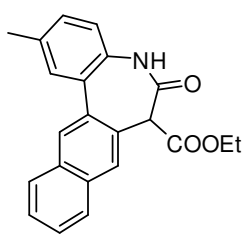

White solid (28 mg, $81 \%$ yield); Mp: $195-196{ }^{\circ} \mathrm{C} ;{ }^{1} \mathrm{H}$ NMR (400 $\left.\mathrm{MHz}, \mathrm{CDCl}_{3}\right), \delta(\mathrm{ppm}): 8.54(\mathrm{~s}, 1 \mathrm{H}), 8.02(\mathrm{~s}, 1 \mathrm{H}), 7.92-7.84(\mathrm{~m}, 3$ H), 7.54-7.49 (m, $3 \mathrm{H}), 7.17$ (dd, $J=8.0 \mathrm{~Hz}, 1.6 \mathrm{~Hz}, 1 \mathrm{H}), 7.01$ (d, $J$ $=8.0 \mathrm{~Hz}, 1 \mathrm{H}), 4.87(\mathrm{~d}, J=0.8 \mathrm{~Hz}, 1 \mathrm{H}), 3.83-3.74(\mathrm{~m}, 2 \mathrm{H}), 2.41(\mathrm{~s}$,

$3 \mathrm{H}), 0.83(\mathrm{t}, J=7.0 \mathrm{~Hz}, 3 \mathrm{H}) ;{ }^{13} \mathrm{C}$ NMR (100 MHz, $\left.\mathrm{CDCl}_{3}\right), \delta(\mathrm{ppm}): 170.27,167.42$, $135.32,134.53,133.30,132.98,132.38,131.95,131.71,130.84,129.87,129.06$, 128.87, 127.99, 127.57, 126.75, 126.67, 122.14, 61.68, 60.35, 20.96, 13.68 ; HR-MS (EI-TOF) $\left(\mathrm{M}^{+}\right)$calculated for $\mathrm{C}_{22} \mathrm{H}_{19} \mathrm{NO}_{3} 345.1365$, found 345.1365.

Methyl 6-oxo-6,7-dihydro-5H-dibenzo[b,d]azepine-7-carboxylate 3p

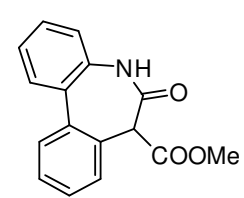

Light yellow solid (18.1 mg, $68 \%$ yield); Mp: 207-208 ${ }^{\circ} \mathrm{C} ;{ }^{1} \mathrm{H}$ NMR (400 MHz, d $\left.{ }_{6}-\mathrm{DMSO}\right), \delta(\mathrm{ppm}): 10.23(\mathrm{~s}, 1 \mathrm{H}), 7.61(\mathrm{~d}, J=7.2 \mathrm{~Hz}, 1$ $\mathrm{H})$, 7.57-7.55 (m, $1 \mathrm{H})$, 7.53-7.46 (m, $3 \mathrm{H})$, 7.40-7.36 (m, $1 \mathrm{H})$, 7.26-7.22 (m, $1 \mathrm{H}), 7.15(\mathrm{~d}, J=8.0 \mathrm{~Hz}, 1 \mathrm{H}), 4.95(\mathrm{~s}, 1 \mathrm{H}), 3.14(\mathrm{~s}, 3 \mathrm{H}) ;{ }^{13} \mathrm{C}$ NMR (100 MHz, d $\mathrm{d}_{6}$-DMSO), $\delta$ (ppm): 169.49, 168.20, 136.77, 135.72, 134.30, 131.46, $130.31,130.05,129.57,129.14,129.02,128.80,125.03,122.41,59.28,52.35$; HR-MS (EI-TOF) $\left(\mathrm{M}^{+}\right)$calculated for $\mathrm{C}_{16} \mathrm{H}_{13} \mathrm{NO}_{3}$ 267.0895, found 267.0897.

\section{Isopropyl 6-oxo-6,7-dihydro-5H-dibenzo[b,d]azepine-7-carboxylate 3q}

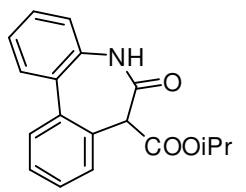

White solid (21 mg, $71 \%$ yield); Mp: 189-190 ${ }^{\circ} \mathrm{C} ;{ }^{1} \mathrm{H}$ NMR (400 
$\left.\mathrm{MHz}, \mathrm{CDCl}_{3}\right), \delta(\mathrm{ppm}): 8.56(\mathrm{~s}, 1 \mathrm{H}), 7.61-7.56(\mathrm{~m}, 2 \mathrm{H}), 7.50-7.41(\mathrm{~m}, 2 \mathrm{H})$, 7.37-7.33 (m, $2 \mathrm{H}), 7.25-7.23(\mathrm{~m}, 1 \mathrm{H}), 7.10(\mathrm{~d}, J=7.6 \mathrm{~Hz}, 1 \mathrm{H}), 4.71(\mathrm{~s}, 1 \mathrm{H})$, 4.64-4.55 (m, $1 \mathrm{H}), 0.92(\mathrm{~d}, J=6.4 \mathrm{~Hz}, 3 \mathrm{H}), 0.76(\mathrm{~d}, J=6.4 \mathrm{~Hz}, 3 \mathrm{H}) ;{ }^{13} \mathrm{C}$ NMR $(100$ $\left.\mathrm{MHz}, \mathrm{CDCl}_{3}\right), \delta(\mathrm{ppm}): 170.59,166.63,136.31,134.56,133.48,131.70,130.24$, $130.18,129.56,128.93,128.81,128.71,125.35,121.99,69.52,60.29,21.24,21.06$; HR-MS (EI-TOF) $\left(\mathrm{M}^{+}\right)$calculated for $\mathrm{C}_{18} \mathrm{H}_{17} \mathrm{NO}_{3} 295.1208$, found 295.1212.

\section{Benzyl 9-chloro-6-oxo-6,7-dihydro-5H-dibenzo[b,d]azepine-7-carboxylate $\mathbf{3 r}$}

White solid (32.8 mg, $87 \%$ yield); Mp: 132-133 ${ }^{\circ} \mathrm{C} ;{ }^{1} \mathrm{H}$ NMR (400

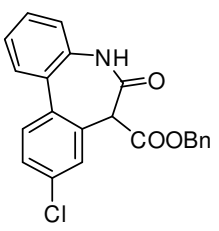
$\left.\mathrm{MHz}, \mathrm{CDCl}_{3}\right), \delta(\mathrm{ppm}): 8.63(\mathrm{~s}, 1 \mathrm{H}), 7.42-7.36(\mathrm{~m}, 3 \mathrm{H}), 7.30(\mathrm{~d}, J=$ $2.0 \mathrm{~Hz}, 1 \mathrm{H}), 7.19-7.08$ (m, $5 \mathrm{H}), 6.93$ (d, $J=6.8 \mathrm{~Hz}, 2 \mathrm{H}), 6.88$ (d, $J$ $=7.6 \mathrm{~Hz}, 1 \mathrm{H}), 4.66-4.60(\mathrm{~m}, 3 \mathrm{H}) ;{ }^{13} \mathrm{C} \mathrm{NMR}\left(100 \mathrm{MHz}, \mathrm{CDCl}_{3}\right), \delta$ (ppm): 169.70, 166.41, 134.97, 134.70, 134.61, 134.39, 134.20, 130.77, 130.40, $129.99,129.76,129.35,129.09,128.45,128.41,128.33,125.55,121.98,67.41,59.55$; HR-MS (EI-TOF) $\left(\mathrm{M}^{+}\right)$calculated for $\mathrm{C}_{22} \mathrm{H}_{16} \mathrm{ClNO}_{3} 377.0819$, found 377.0821 .

\section{Benzyl 6-oxo-6,7-dihydro-5H-dibenzo[b,d]azepine-7-carboxylate 3s}

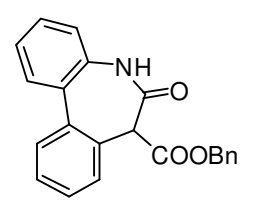

Light yellow solid (32.1 mg, $94 \%$ yield). Mp: $132-133{ }^{\circ} \mathrm{C} ;{ }^{1} \mathrm{H}$ NMR (400 MHz, $\left.\mathrm{CDCl}_{3}\right), \delta(\mathrm{ppm}): 8.86(\mathrm{~s}, 1 \mathrm{H}), 7.55$ (d, $J=7.6 \mathrm{~Hz}$, 1H), 7.51-7.41 (m, $3 \mathrm{H}), 7.36(\mathrm{~d}, J=7.6 \mathrm{~Hz}, 1 \mathrm{H}), 7.25-7.16(\mathrm{~m}, 5$ H), 7.00-6.96 (m, $3 \mathrm{H}) 4.78(\mathrm{~d}, J=0.8 \mathrm{~Hz}, 1 \mathrm{H}), 4.73-4.66(\mathrm{~m}, 2 \mathrm{H}) ;{ }^{13} \mathrm{C}$ NMR $(100$ $\left.\mathrm{MHz}, \mathrm{CDCl}_{3}\right), \delta(\mathrm{ppm}): 170.39,167.02,136.40,134.84,134.34,133.16,131.47$, $130.16,129.94,129.54,129.00,128.94,128.74,128.40,128.29,128.20,125.37$, 121.91, 67.19, 59.99; HR-MS (EI-TOF) $\left(\mathrm{M}^{+}\right)$calculated for $\mathrm{C}_{22} \mathrm{H}_{17} \mathrm{NO}_{3} 343.1208$, found 343.1202 .

Tert-butyl 6-oxo-6,7-dihydro-5H-dibenzo[b,d]azepine-7-carboxylate 3t

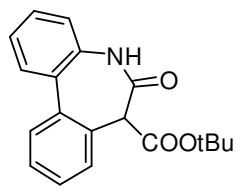

White solid (17.6 mg, $57 \%$ yield); Mp: 206-207 ${ }^{\circ} \mathrm{C} ;{ }^{1} \mathrm{H}$ NMR (400 $\left.\mathrm{MHz}, \mathrm{CDCl}_{3}\right), \delta$ (ppm): 8.69 (s, $\left.1 \mathrm{H}\right), 7.62(\mathrm{dd}, J=7.6 \mathrm{~Hz}, 1.2 \mathrm{~Hz}$, $1 \mathrm{H}), 7.56(\mathrm{dd}, J=7.6 \mathrm{~Hz}, 1.6 \mathrm{~Hz}, 1 \mathrm{H}), 7.48-7.33(\mathrm{~m}, 4 \mathrm{H})$, 7.27-7.23 (m, $1 \mathrm{H}), 7.13(\mathrm{dd}, J=8.0 \mathrm{~Hz}, 0.8 \mathrm{~Hz}, 1 \mathrm{H})), 4.69(\mathrm{~d}, J=1.6 \mathrm{~Hz}, 1 \mathrm{H})$, 
1.02 (s, $9 \mathrm{H}) ;{ }^{13} \mathrm{C}$ NMR (100 MHz, $\left.\mathrm{CDCl}_{3}\right), \delta$ (ppm): 171.09, 166.13, 136.23, 134.87, $133.85,131.74,130.30,129.56,128.92,128.70,128.65,125.25,121.94,82.53,61.15$, 27.21; HR-MS (EI-TOF) $\left(\mathrm{M}^{+}\right)$calculated for $\mathrm{C}_{19} \mathrm{H}_{19} \mathrm{NO}_{3} 309.1365$, found 309.1365.

\section{Ethyl 5-oxo-5,6-dihydro-4H-benzo[b]furo[3,2-d]azepine-4-carboxylate 3u}

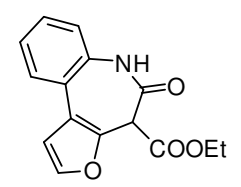

White solid (21.6 mg, $80 \%$ yield); Mp: $141-148{ }^{\circ} \mathrm{C} ;{ }^{1} \mathrm{H}$ NMR (400 $\left.\mathrm{MHz}, \mathrm{CDCl}_{3}\right), \delta$ (ppm): 9.52 (s, $\left.1 \mathrm{H}\right), 7.51-7.49$ (m, $\left.2 \mathrm{H}\right), 7.31-7.27$ (m, $1 \mathrm{H}), 7.23-7.18(\mathrm{~m}, 2 \mathrm{H}), 6.73(\mathrm{~d}, J=1.6 \mathrm{~Hz}, 1 \mathrm{H})), 5.04(\mathrm{~d}, J=$ $0.8 \mathrm{~Hz}, 1 \mathrm{H}), 4.01-3.92(\mathrm{~m}, 2 \mathrm{H}), 0.92(\mathrm{t}, J=7.2 \mathrm{~Hz}, 3 \mathrm{H}) ;{ }^{13} \mathrm{C}$ NMR $(100 \mathrm{MHz}$, $\left.\mathrm{CDCl}_{3}\right), \delta(\mathrm{ppm}): 167.35,165.43,143.62,142.36,133.27,128.01,127.54,125.00$, $122.35,121.88,120.30,109.79,62.16,53.40,13.71 ;$ HR-MS (EI-TOF) $\left(\mathrm{M}^{+}\right)$ calculated for $\mathrm{C}_{15} \mathrm{H}_{13} \mathrm{NO}_{4} 271.0845$, found 271.0843 .

Ethyl 5-oxo-5,6-dihydro-4H-benzo[b]thieno[3,2-d]azepine-4-carboxylate 3v

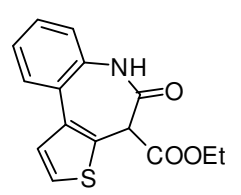

Light yellow solid (13.7 mg, $48 \%$ yield). Mp: $165-166{ }^{\circ} \mathrm{C} ;{ }^{1} \mathrm{H}$ NMR $\left(400 \mathrm{MHz}, \mathrm{CDCl}_{3}\right), \delta(\mathrm{ppm}): 8.84(\mathrm{~s}, 1 \mathrm{H}), 7.60(\mathrm{dd}, J=7.8 \mathrm{~Hz}, 1.4$ $\mathrm{Hz}, 1 \mathrm{H}), 7.36(\mathrm{~d}, J=5.2 \mathrm{~Hz}, 1 \mathrm{H}), 7.33(\mathrm{dd}, J=8.0 \mathrm{~Hz}, 0.8 \mathrm{~Hz}, 1 \mathrm{H})$, 7.28-7.23 (m, $2 \mathrm{H}), 7.17$ (dd, $J=7.8 \mathrm{~Hz}, 1.0 \mathrm{~Hz}, 1 \mathrm{H}), 4.59$ (s, $1 \mathrm{H}), 4.19-4.11$ (m, 2 H), $1.10(\mathrm{t}, J=7.2 \mathrm{~Hz}, 3 \mathrm{H}) ;{ }^{13} \mathrm{C}$ NMR (100 MHz, $\left.\mathrm{CDCl}_{3}\right), \delta$ (ppm): 168.27, 166.88, $136.66,133.92,129.62,128.69,128.40,127.21,126.68,125.95,125.18,122.27$, 62.06, 51.69, 13.89; HR-MS (EI-TOF) $\left(\mathrm{M}^{+}\right)$calculated for $\mathrm{C}_{15} \mathrm{H}_{13} \mathrm{NO}_{3} \mathrm{~S}$ 287.0616, found 287.0617 .

\section{Primary Mechanistic Study.}

(1) Control Experiment.

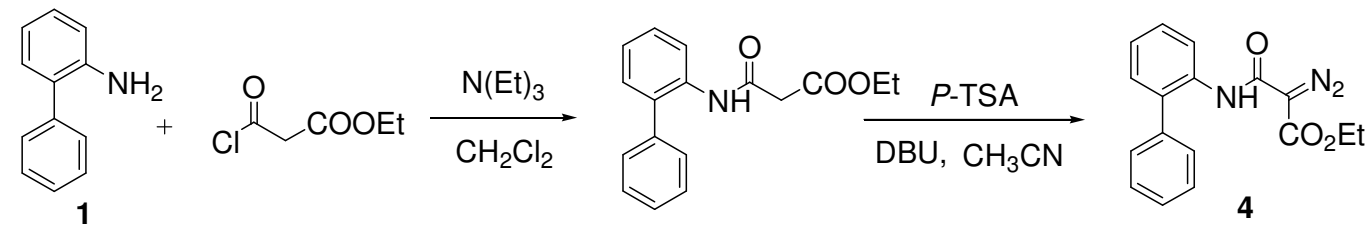

The diazo compound 4 was obtained as a white solid according to previous method. ${ }^{3,4} \mathrm{Mp}: 213-215{ }^{\circ} \mathrm{C}$; ${ }^{1} \mathrm{H}$ NMR (400 MHz, $\mathrm{d}_{6}$-DMSO), $\delta$ (ppm): 7.50-7.47 (m, $3 \mathrm{H}), 7.29-7.26$ (m, $6 \mathrm{H}), 4.14-4.11(\mathrm{~m}, 2 \mathrm{H}), 1.24(\mathrm{t}, J=6.8 \mathrm{~Hz}, 3 \mathrm{H}) .{ }^{13} \mathrm{C}$ NMR $(100$ 
MHz, $\mathrm{d}_{6}$-DMSO), $\delta$ (ppm): 164.24, 162.27, 139.86, 139.36, 134.86, 130.97, 129.62, 129.16, 128.84, 128.51, 128.23, 127.39, 115.18, 58.51, 15.14. HR-MS (ESI-TOF) $(\mathrm{M}+\mathrm{H})^{+}$calculated for $\mathrm{C}_{17} \mathrm{H}_{15} \mathrm{~N}_{3} \mathrm{O}_{3} 310.1183$, found 310.1191 .<smiles>CCOC(=O)C(=N)C(=O)Nc1ccccc1-c1ccccc1</smiles>

$4(0.10 \mathrm{~mol})$

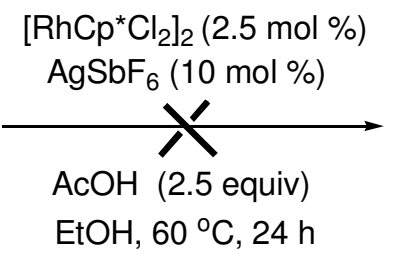

$\mathrm{EtOH}, 60^{\circ} \mathrm{C}, 24 \mathrm{~h}$

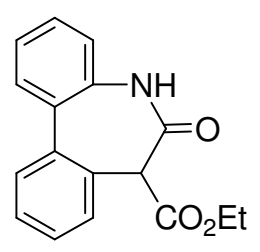

3a

(2) Isotope Kinetic Effect Experiment<smiles>[2H]c1c([2H])c([2H])c(Br)c([2H])c1[2H]</smiles><smiles>[2H]c1c([2H])c([2H])c([12Br])c(Br)c1[2H]</smiles>

1) $\mathrm{B}(\mathrm{OMe})_{3}, \mathrm{THF}$, $\frac{-78{ }^{\circ} \mathrm{C}}{\text { 2) } 10 \% \mathrm{HCl}}$<smiles>[2H]c1c([2H])c([2H])c(B(O)O)c([2H])c1[2H]</smiles>

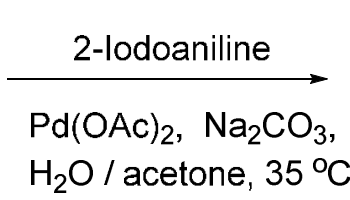<smiles>[2H]c1c([2H])c([2H])c(-c2ccccc2N)c([2H])c1[2H]</smiles>

$1 \mathrm{a}-\mathrm{D}_{5}$

The compound 1a- $\mathrm{D}_{5}$ was obtained as a yellow liquid according to previous method. ${ }^{5} \mathrm{H}$ NMR (400 MHz, $\left.\mathrm{CDCl}_{3}\right), \delta$ (ppm): 7.17-7.11 (m, 2H), $6.82(\mathrm{td}, J=1.2$, $7.4 \mathrm{~Hz}, 1 \mathrm{H}$ ), 6.75 (dd, $J=0.8,8.0 \mathrm{~Hz}, 1 \mathrm{H}), 3.56$ (br s, 2H); ${ }^{13} \mathrm{C}$ NMR (100 MHz, $\left.\mathrm{CDCl}_{3}\right), \delta$ (ppm): 143.52, 139.36, 130.48, 128.51, 127.63, 118.68, 115.63; HR-MS (EI-TOF) $\left(\mathrm{M}^{+}\right)$calculated for $\mathrm{C}_{12} \mathrm{H}_{6} \mathrm{D}_{5} \mathrm{~N}$ 174.1205, found 174.1208.<smiles>Nc1ccccc1-c1ccccc1</smiles>

$1 \mathrm{a}(0.20 \mathrm{mmol})$<smiles>[2H]c1c([2H])c([2H])c(-c2ccccc2N)c([2H])c1[2H]</smiles>

$1 \mathrm{a}-\mathrm{D}_{5}(0.20 \mathrm{mmol})$

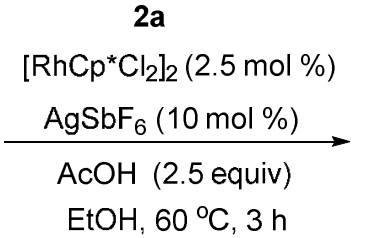

$\mathrm{EtOH}, 60^{\circ} \mathrm{C}, 3 \mathrm{~h}$<smiles>[2H]c1c([2H])c([2H])c2c(c1[2H])-c1ccccc1NC(=O)C2C([O-])OCC</smiles>

3a: $: 3 a-D_{4}=0.80: 0.20$ $k_{H}: k_{D}=4.0$

The rate of 1a:1a-D $\mathrm{D}_{4}$ was from the ${ }^{1} \mathrm{H}$ NMR.

\section{References:}


[1] Stokes, B. J.; Jovanović, B.; Dong, H.; Richert, K. J.; Riell, R. D.; Driver, T. G. J. Org. Chem.

2009, 74, 3225 .

[2] Alt, I. T.; Plietker, B. Angew.Chem. Int.Ed. 2016, 55, 1519.

[3] Gopalakrishnan, B.; Babu, S. A.; Padmavathi, R. Tetrahedron. 2015, 71, 8333

[4] Wang, H. L.; Li, Z.; G. Wang, W. S.; Yang, D. Chem. Commun. 2011, 47, 11336.

[5] Liang, Z.; Ju, L.; Xie, Y.; Huang L.; Zhang, Y. Chem.-Eur. J. 2012, 18, 15816.

\section{X-ray Crystallographic Data of Compound 3e}
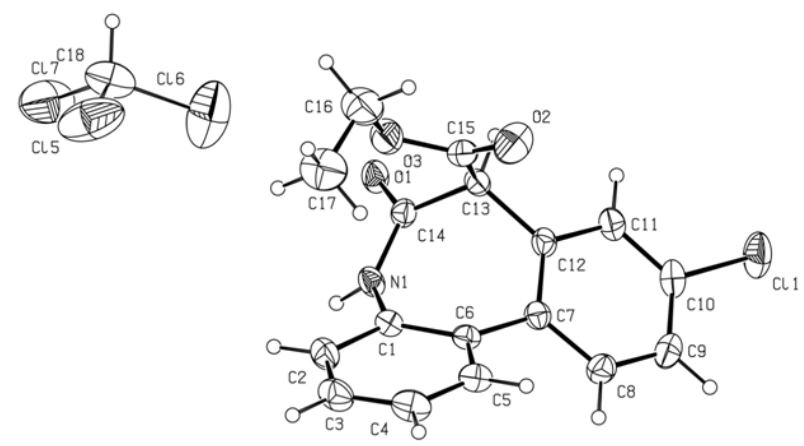

Datablock: 160317_3e_bai1601072

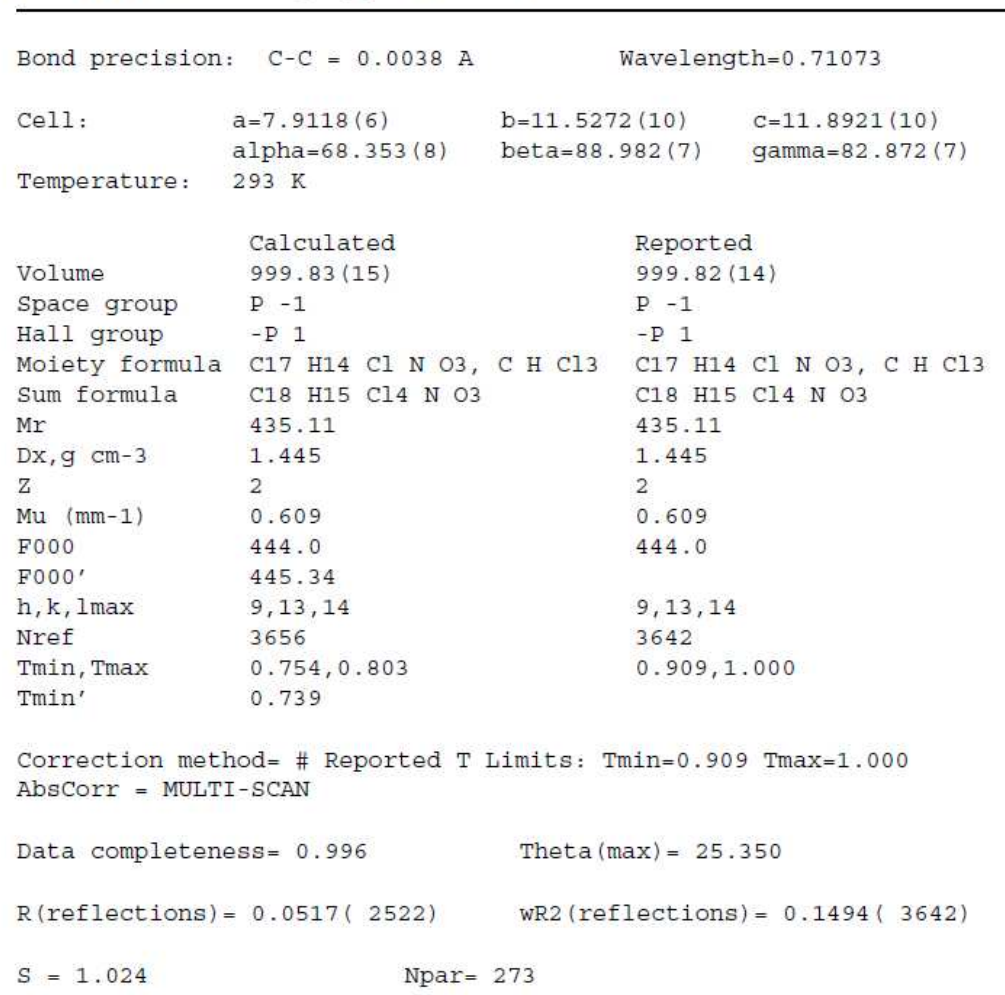


8. ${ }^{1} \mathrm{H}$ NMR, ${ }^{13} \mathrm{C}$ NMR, and HR-MS Spectrum of $\alpha$-Allylated Products.

${ }^{1}$ H NMR Spectrum of ethyl 6-oxo-6,7-dihydro-5H-dibenzo[b,d]azepine-7-carboxylate 3a

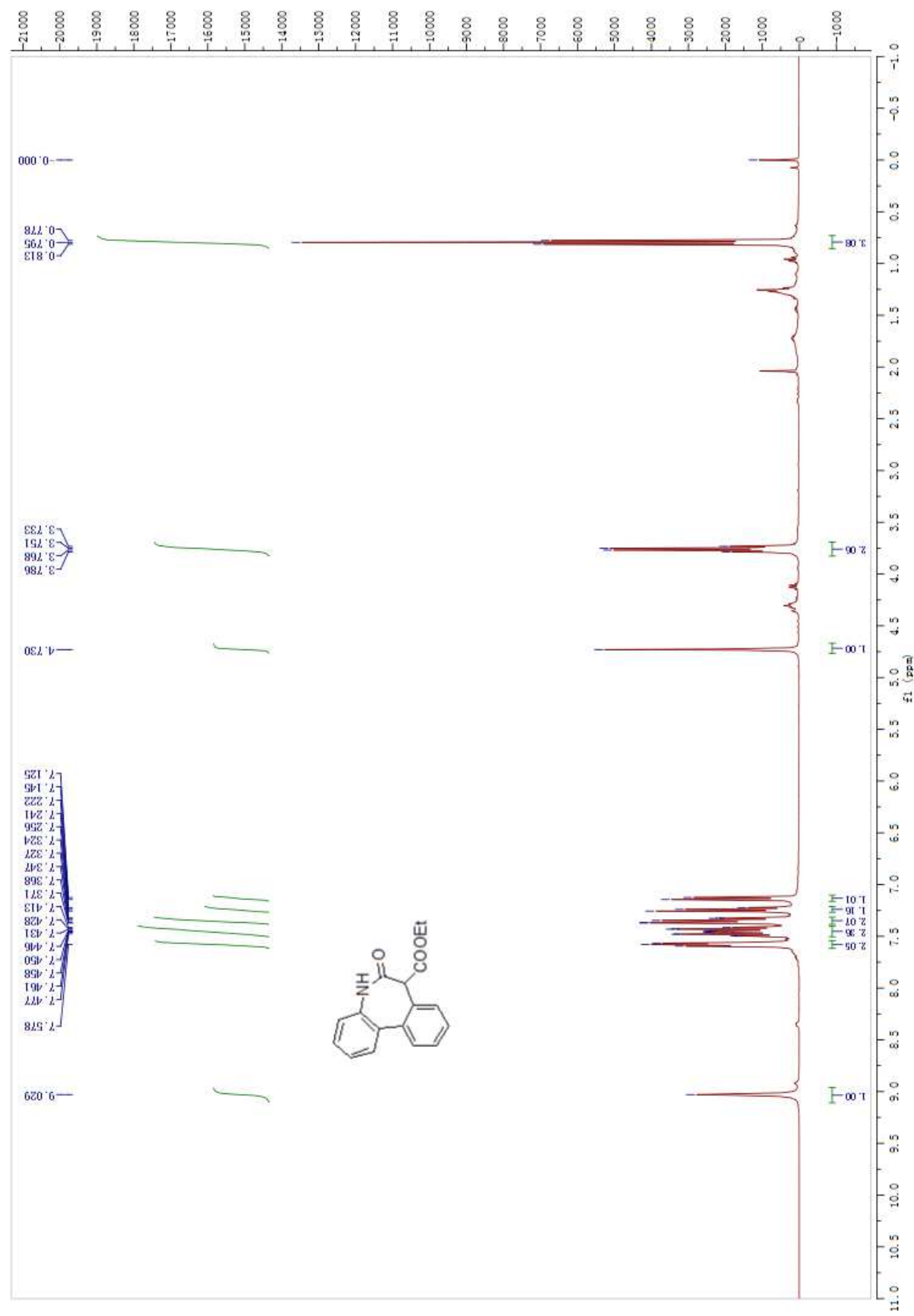


${ }^{13} \mathrm{C}$ NMR Spectrum of ethyl 6-oxo-6,7-dihydro-5H-dibenzo[b,d]azepine-7- carboxy late 3a

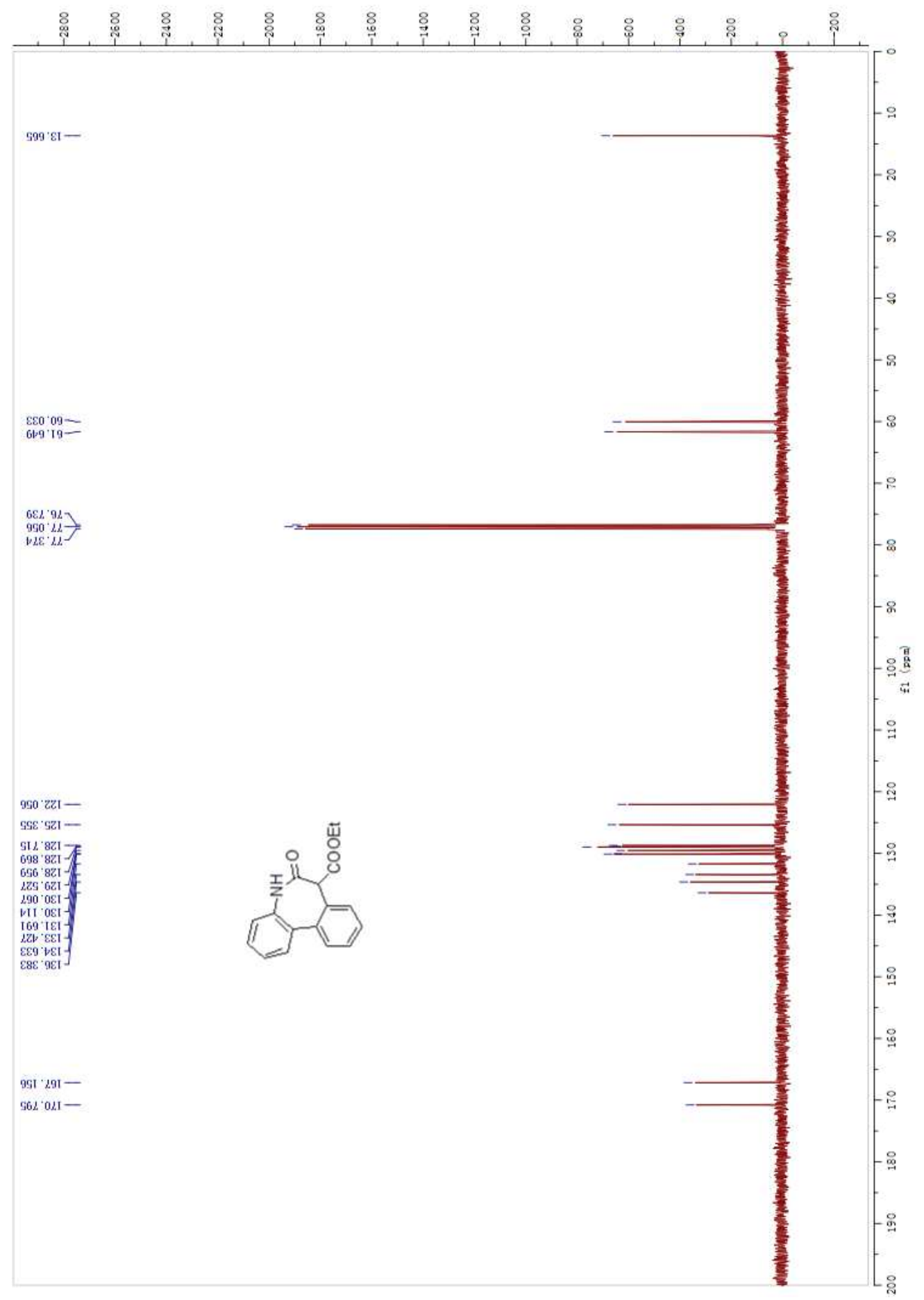


HR-MS (EI) Spectrum of ethyl 6-oxo-6,7-dihydro-5H-dibenzo[b,d]azepine-7carboxylate $\mathbf{3 a}$

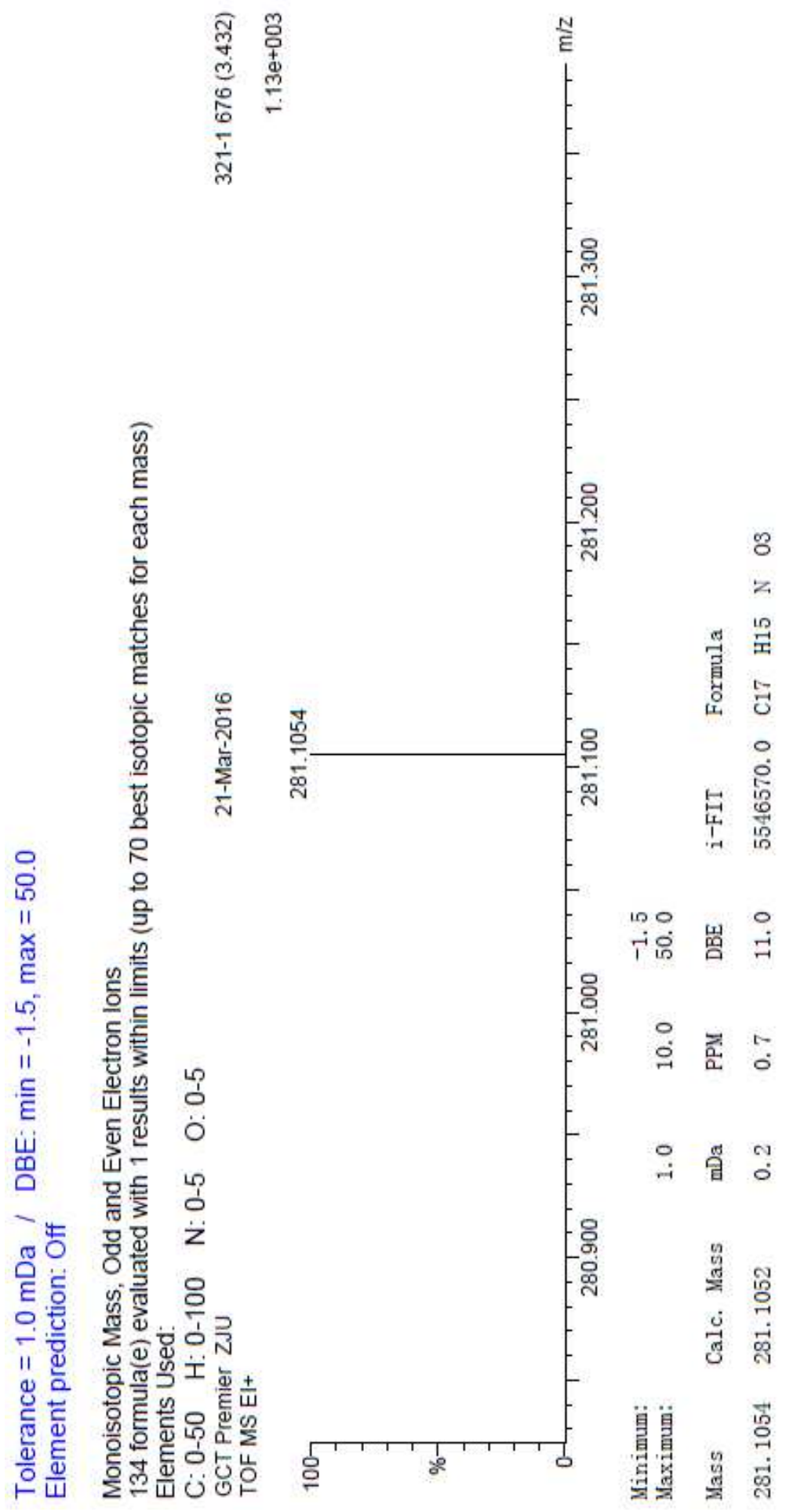


${ }^{1} \mathrm{H}$ NMR Spectrum of ethyl 9-methoxy-6-oxo-6,7-dihydro-5H-dibenzo[b,d]azepine-7carboxylat e $\mathbf{3 b}$

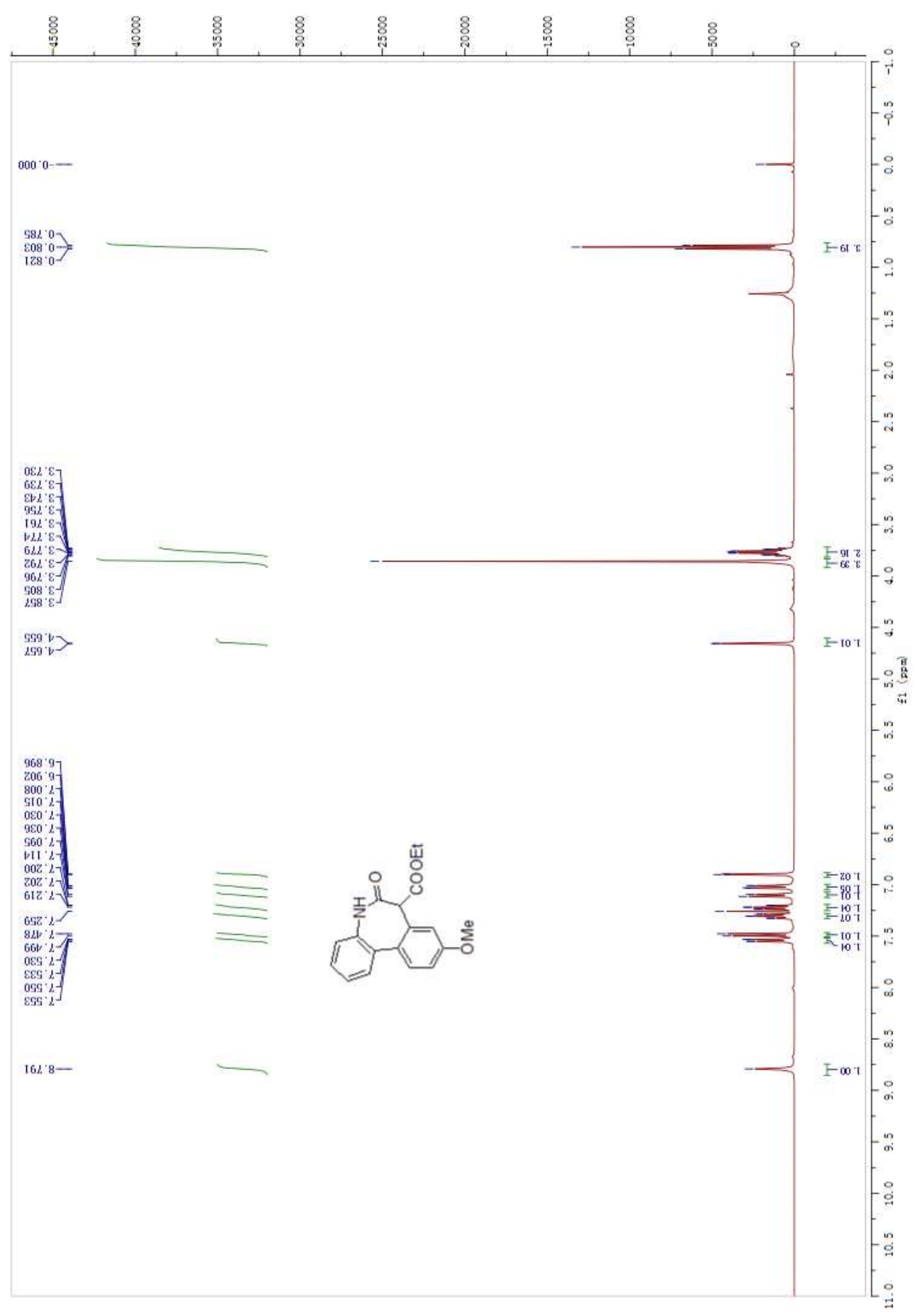


${ }^{13} \mathrm{C}$ NMR Spectrum of ethyl 9-methoxy-6-oxo-6,7-dihydro-5H-dibenzo[b,d]azepine-7 - carboxy late 3b

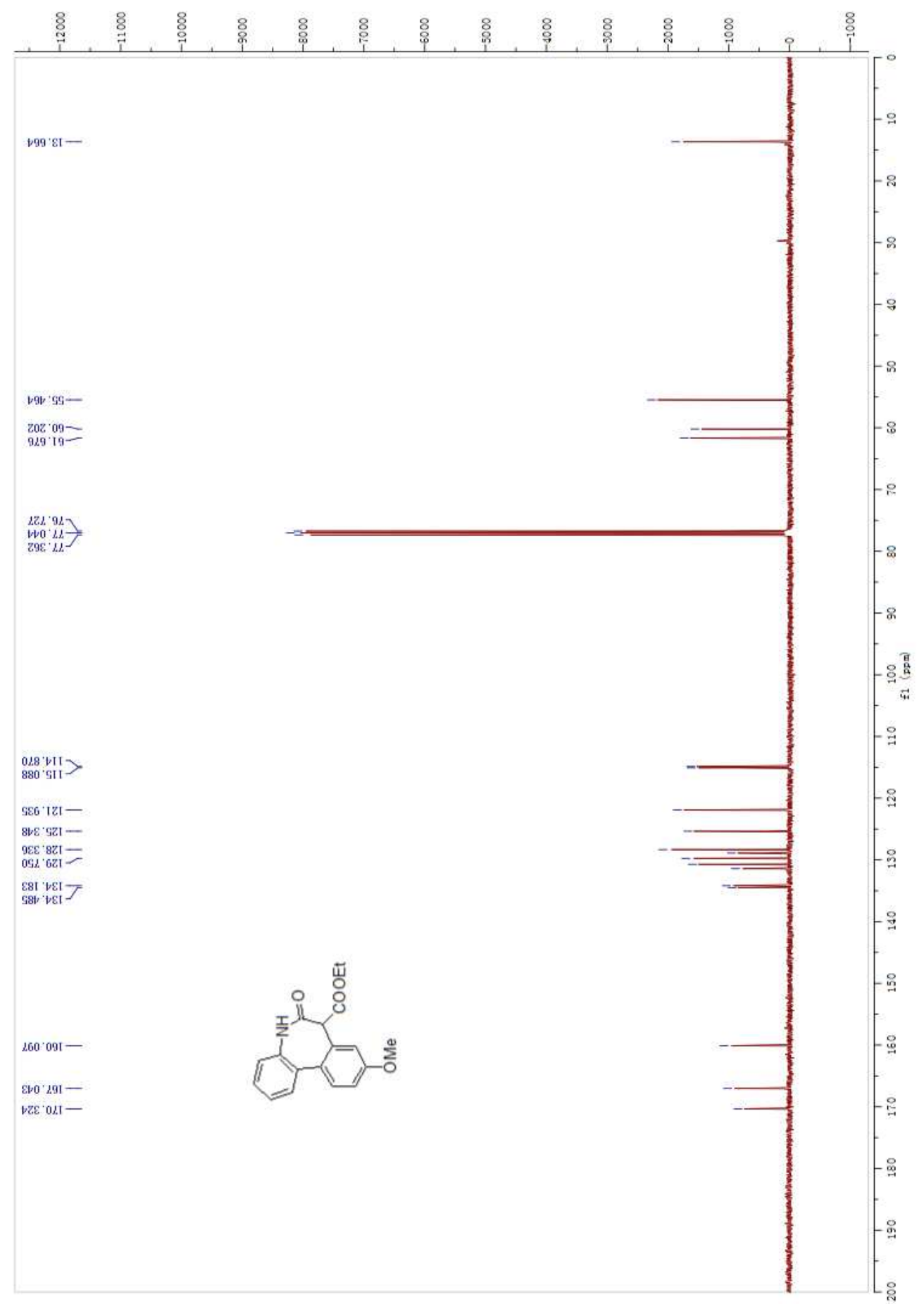


HR-MS (EI) Spectrum of ethyl 9-methoxy-6-oxo-6,7-dihydro-5H-dibenzo[b,d] azepine -7-carboxylate $\mathbf{3 b}$

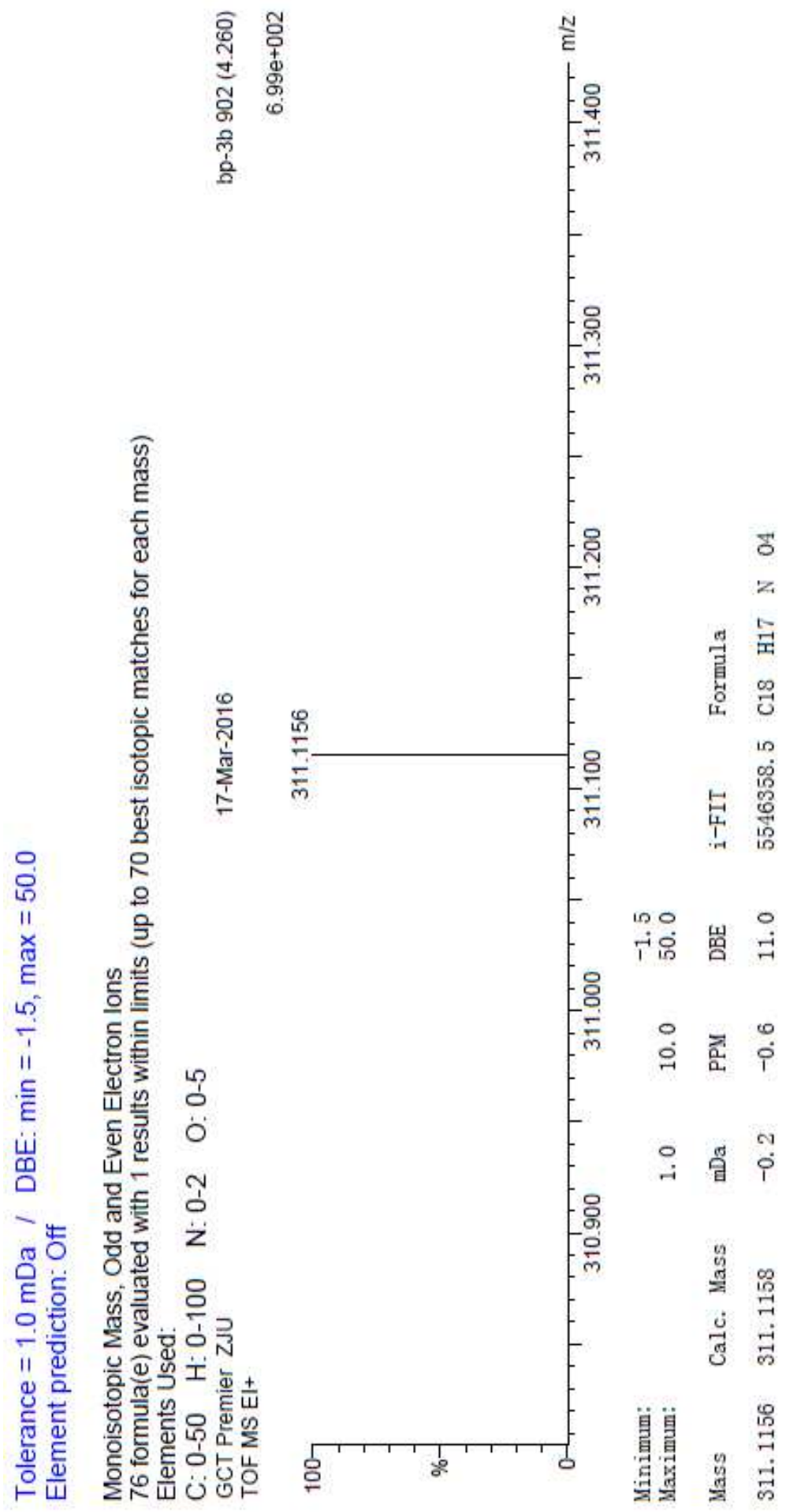


${ }^{1}$ H NMR Spectrum of ethyl 9-methyl-6-oxo-6,7-dihydro-5H-dibenzo[b,d]azepine-7carboxylate $\mathbf{3 c}$

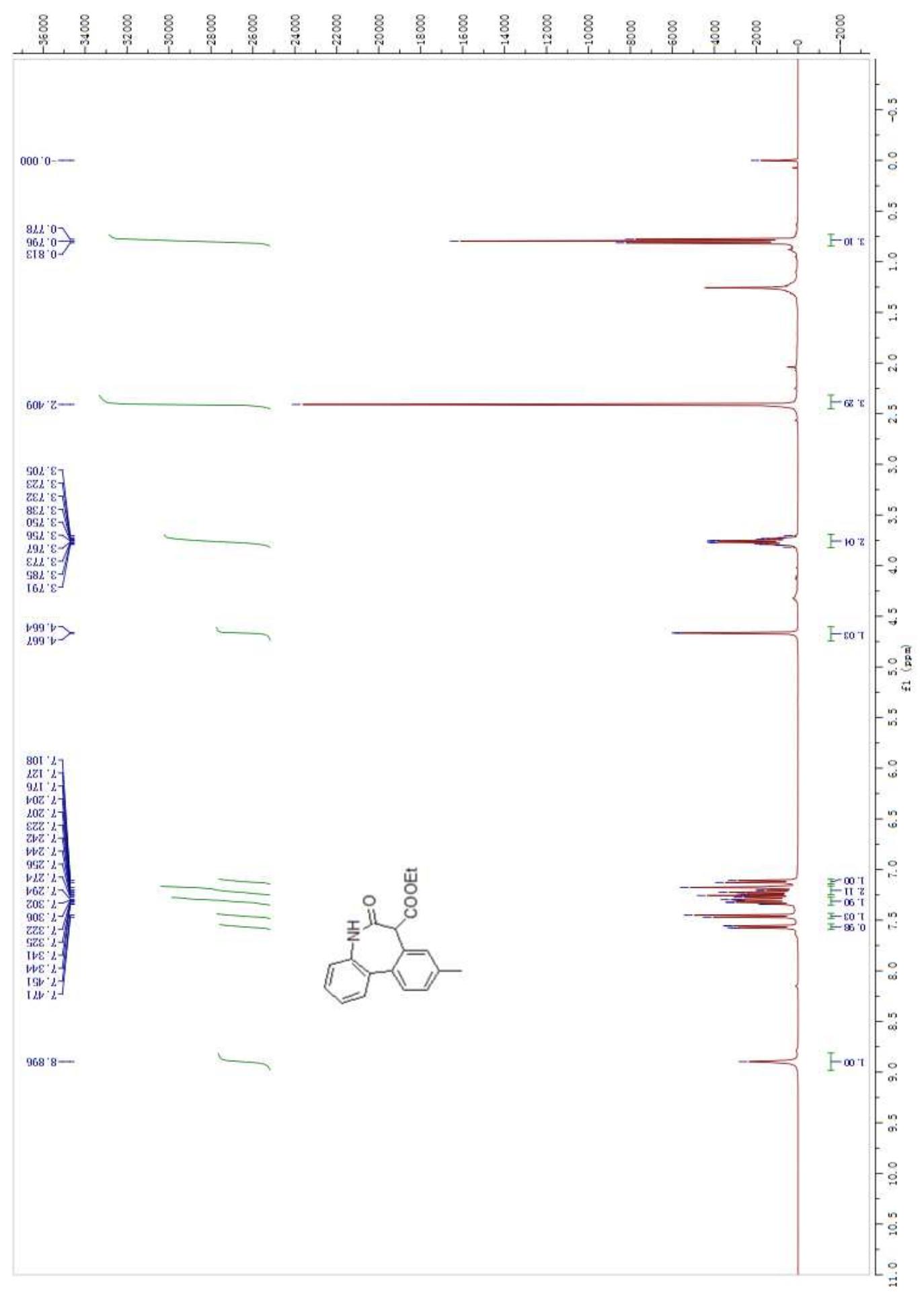


${ }^{13}$ C NMR Spectrum of ethyl 9-methyl-6-oxo-6,7-dihydro-5H-dibenzo[b,d]azepine-7carboxylate $\mathbf{3 c}$

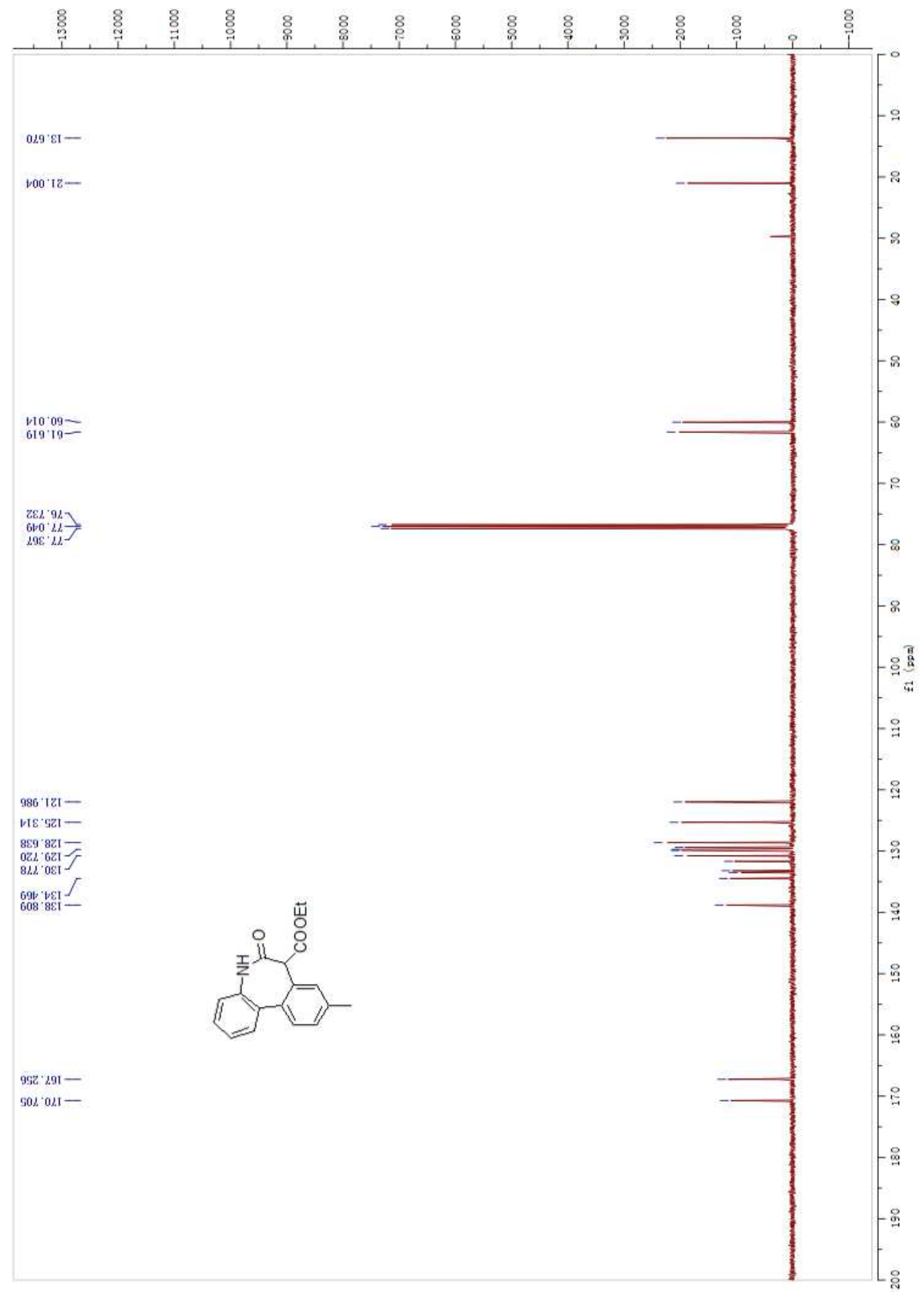


HR-MS (EI) Spectrum of ethyl 9-methyl-6-oxo-6,7-dihydro-5H-dibenzo[b,d]azepine7- carboxylate $\mathbf{3 c}$

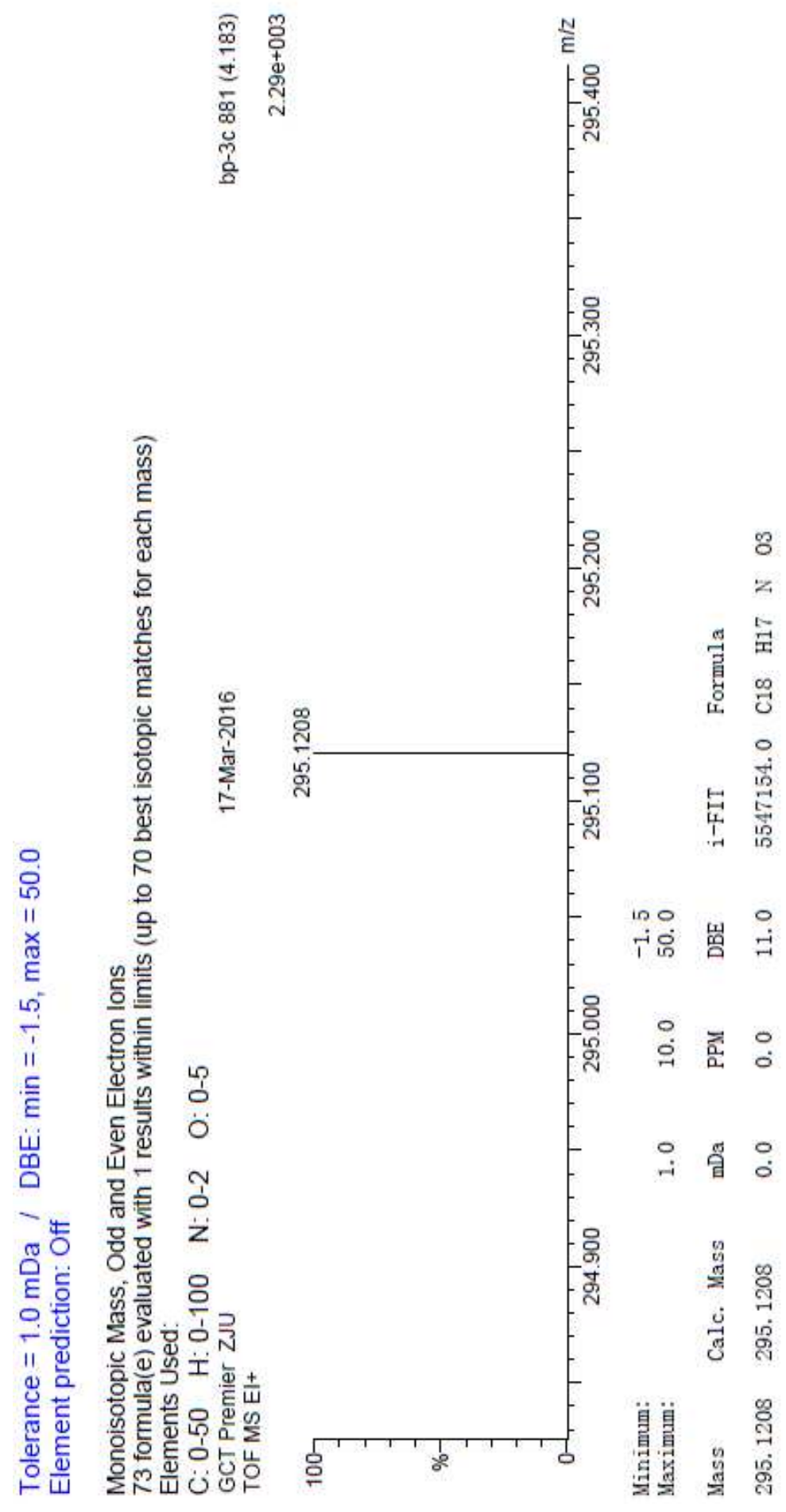


${ }^{1} \mathrm{H}$ NMR Spectrum of ethyl 9-fluoro-6-oxo-6,7-dihydro-5H-dibenzo[b,d]azepine-7carboxylate 3d

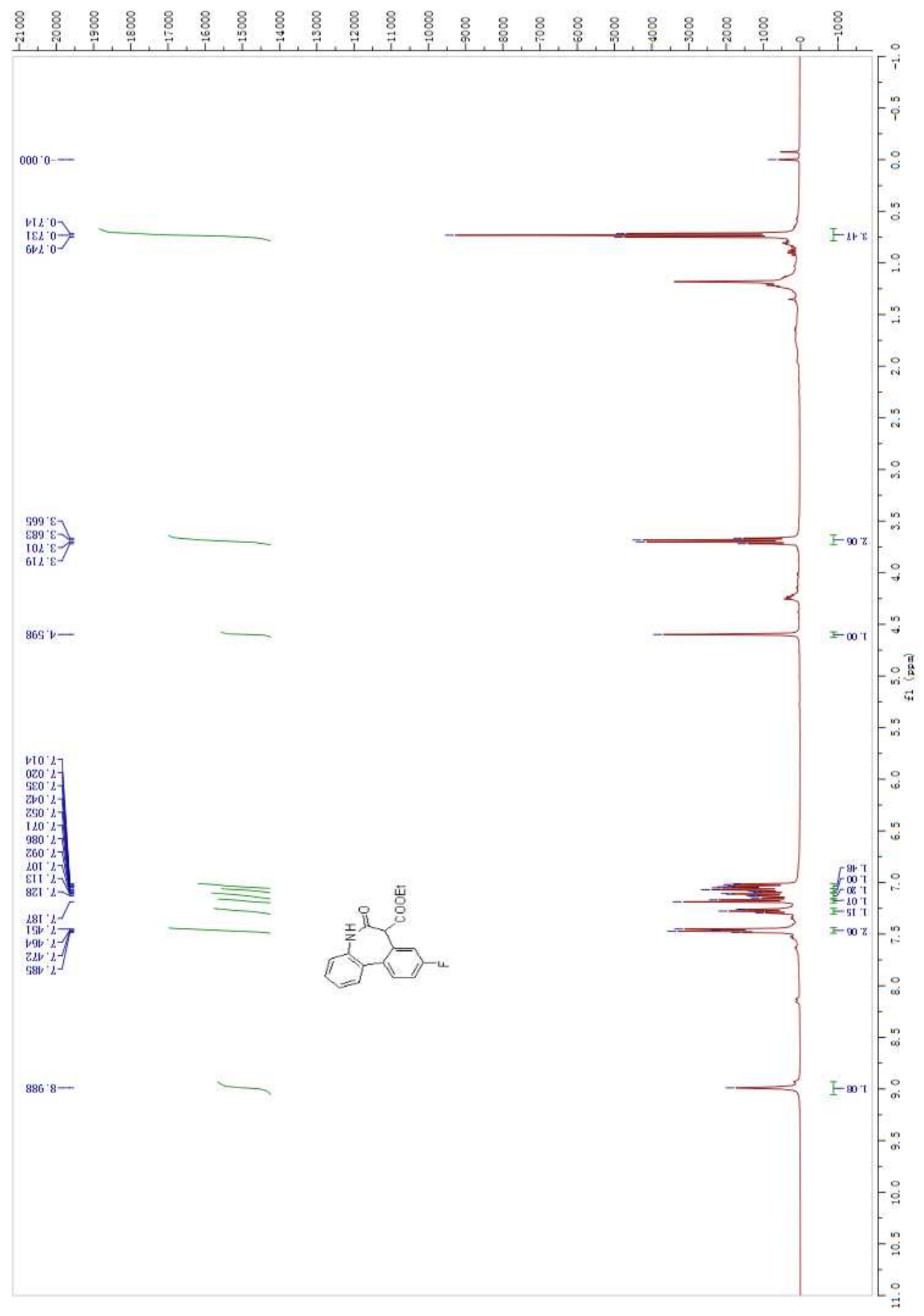


${ }^{13}$ C NMR Spectrum of ethyl 9-fluoro-6-oxo-6,7-dihydro-5H-dibenzo[b,d]azepine-7carboxylate $\mathbf{3 d}$

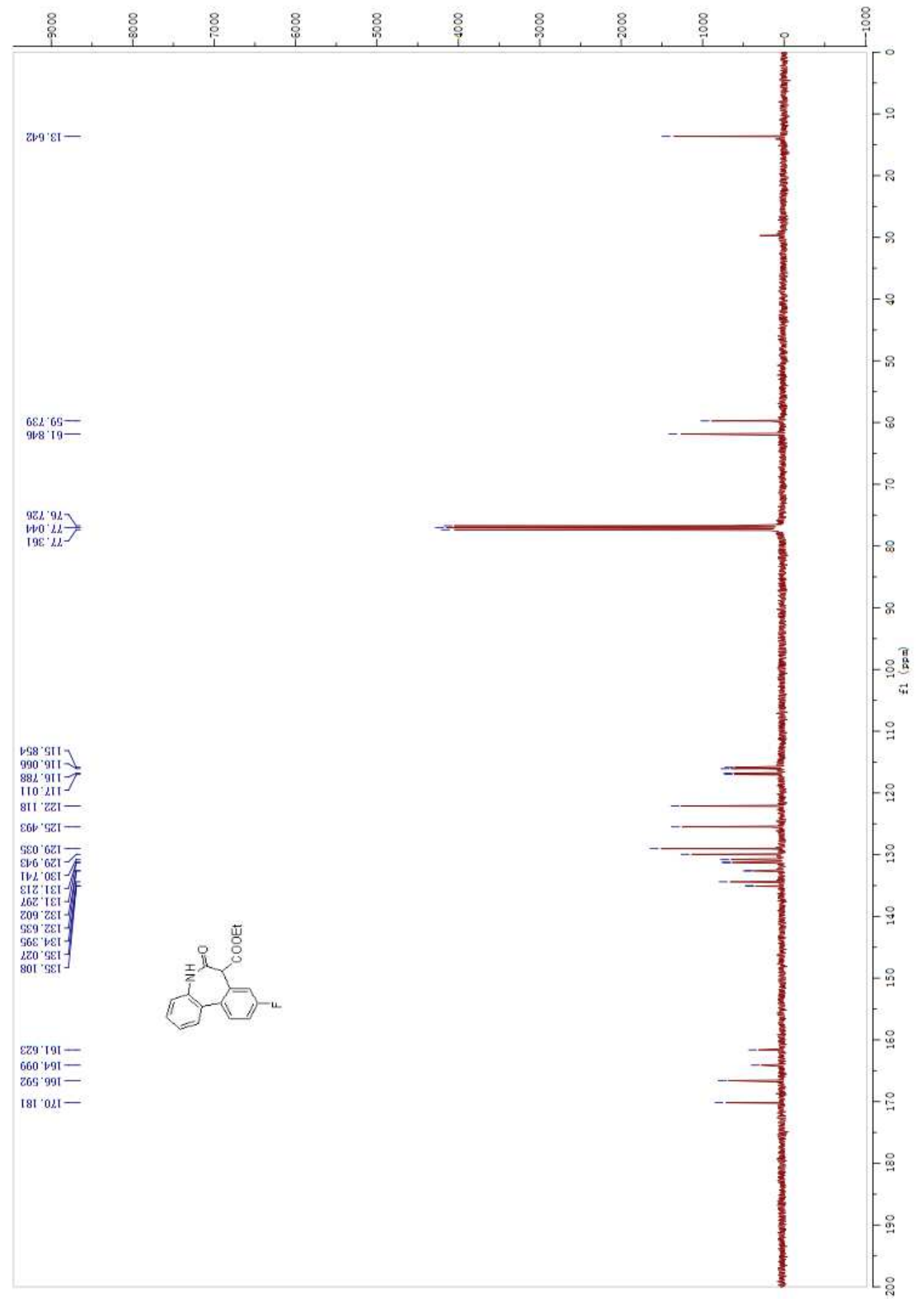


HR-MS (EI) Spectrum of ethyl 9-fluoro-6-oxo-6,7-dihydro-5H-dibenzo[b,d]azepine7 -carboxylate 3d

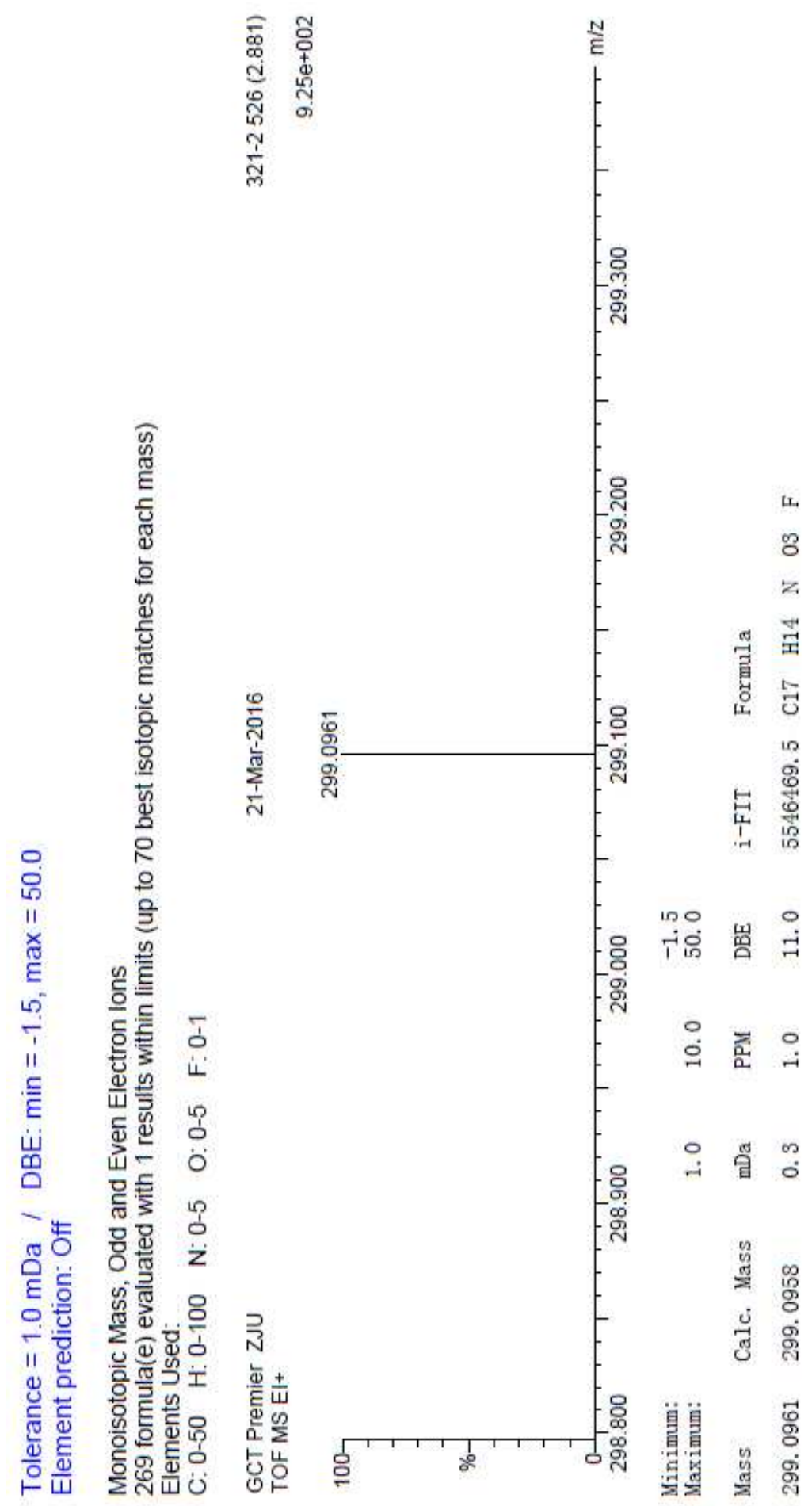


${ }^{1}$ H NMR Spectrum of ethyl 9-chloro-6-oxo-6,7-dihydro-5H-dibenzo[b,d]azepine-7carboxylate $\mathbf{3 e}$

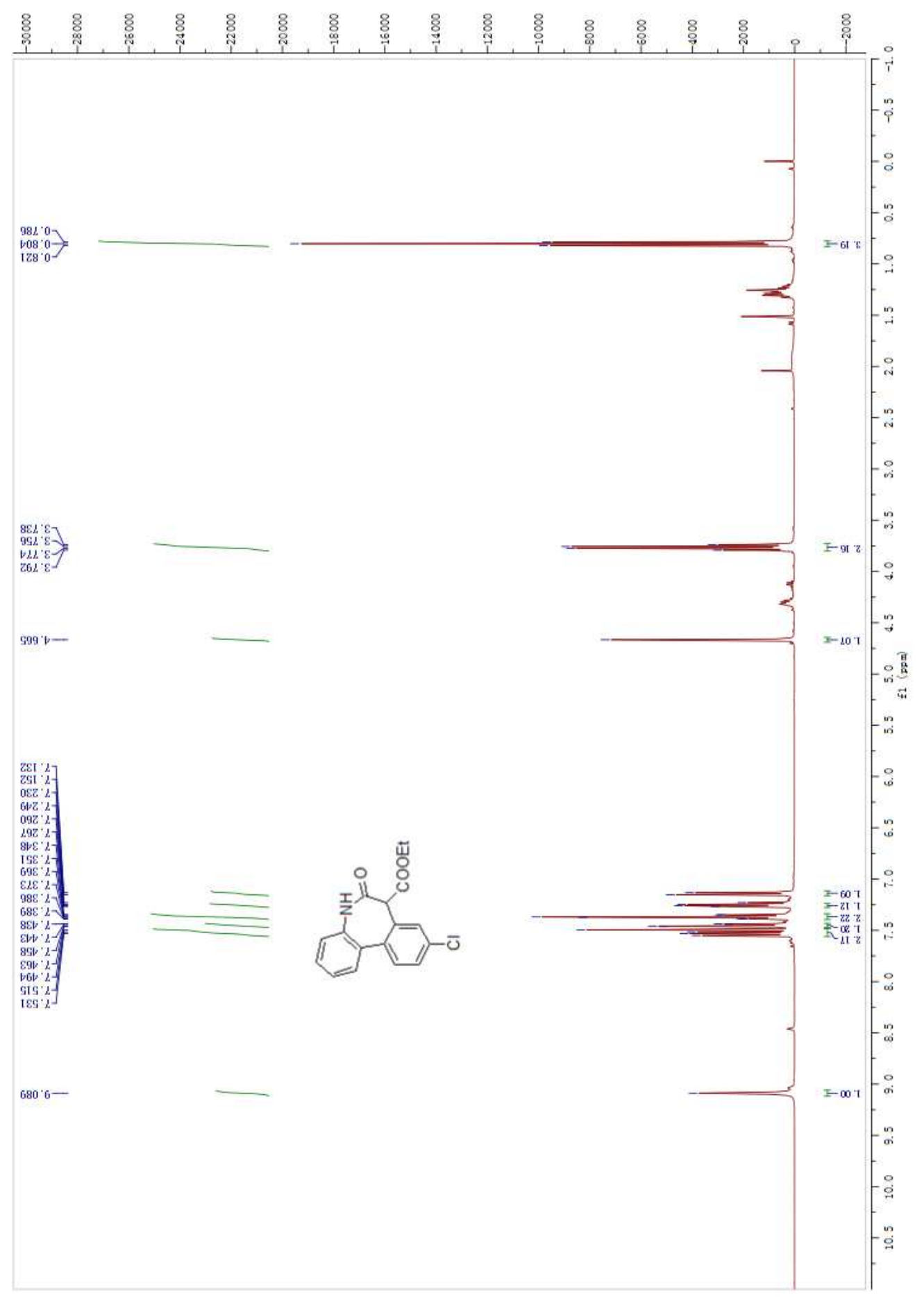


${ }^{13}$ C NMR Spectrum of ethyl 9-chloro-6-oxo-6,7-dihydro-5H-dibenzo[b,d]azepine-7carboxylate $\mathbf{3 e}$

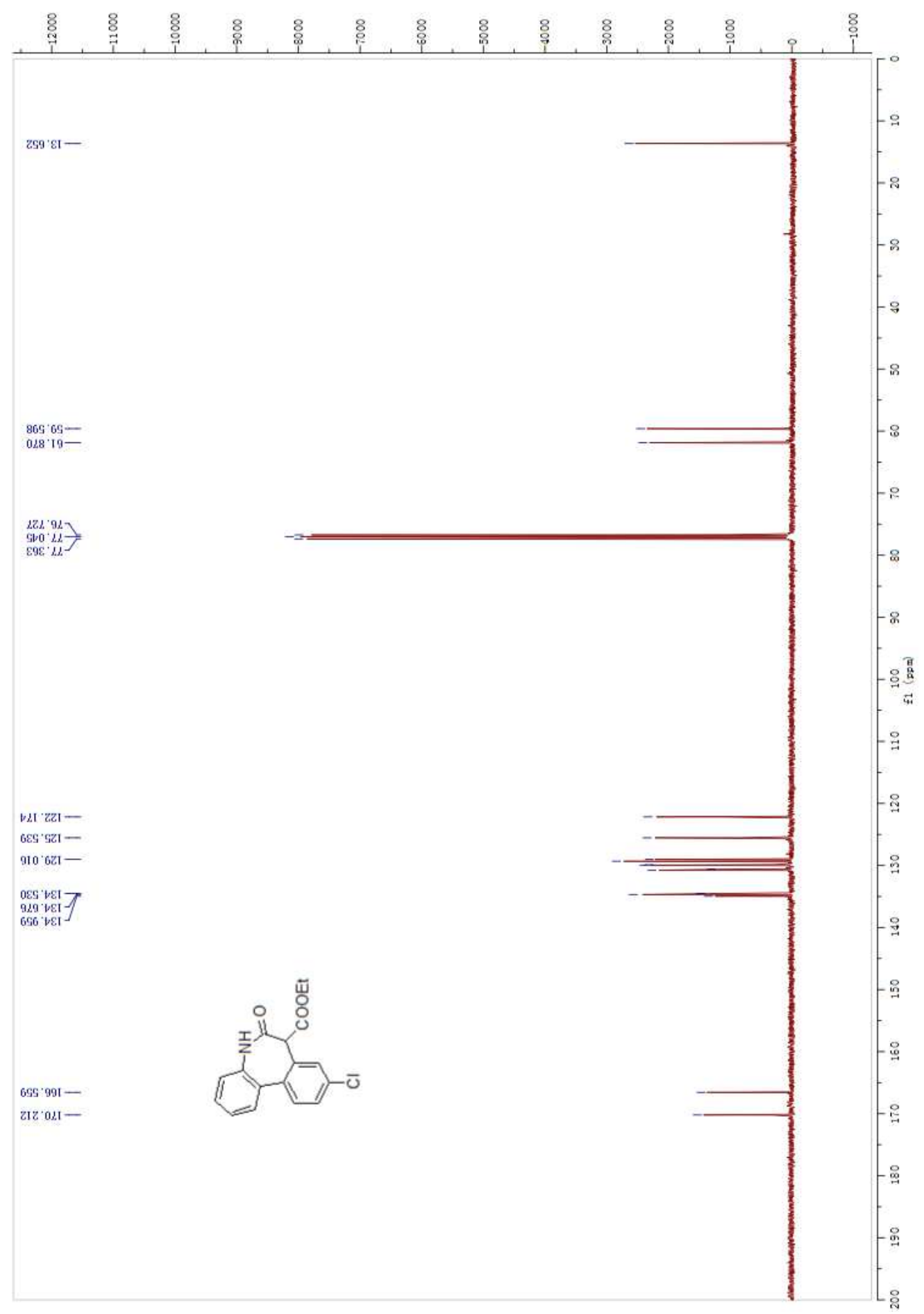


HR-MS (EI) Spectrum of ethyl 9-chloro-6-oxo-6,7-dihydro-5H-dibenzo[b,d]azepine 7- carboxylate $\mathbf{3 e}$

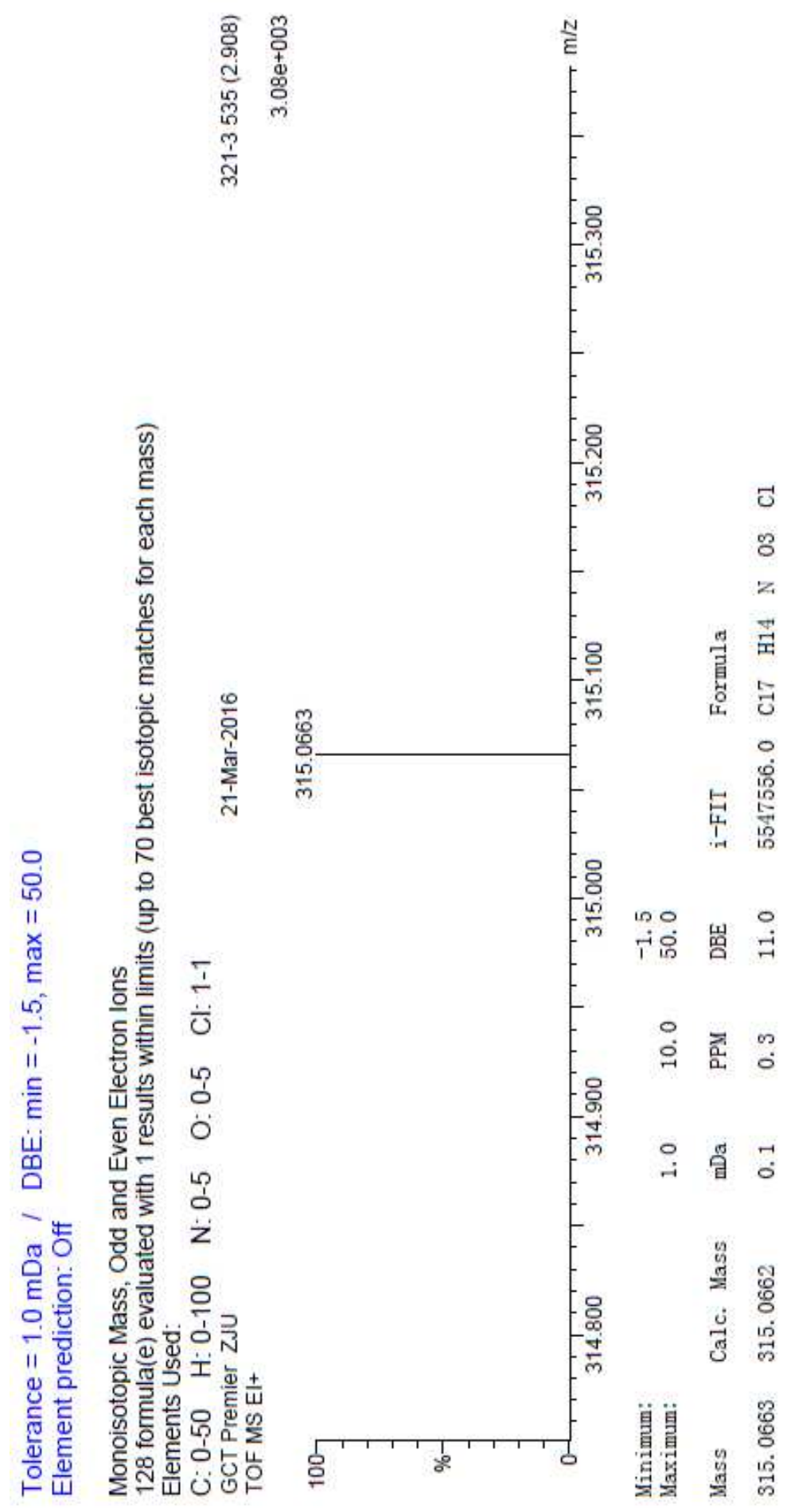


${ }^{1}$ H NMR Spectrum of diethyl 6-oxo-6,7-dihydro-5H-dibenzo[b,d]azepine-7,9-di carboxylate $\mathbf{3 f}$

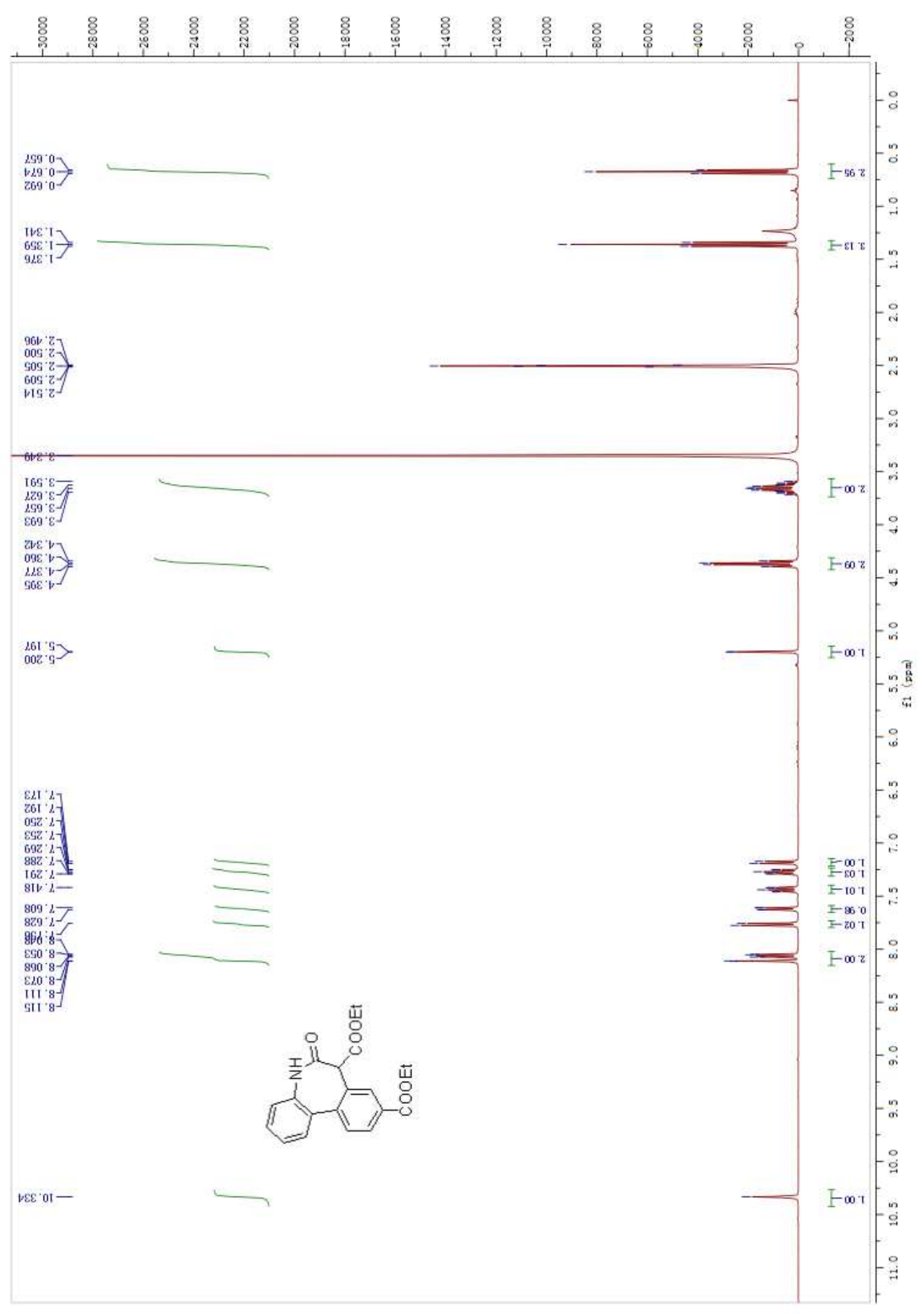


${ }^{13} \mathrm{C}$ NMR Spectrum of diethyl 6-oxo-6,7-dihydro-5H-dibenzo[b,d]azepine-7,9-di carboxylate $\mathbf{3 f}$

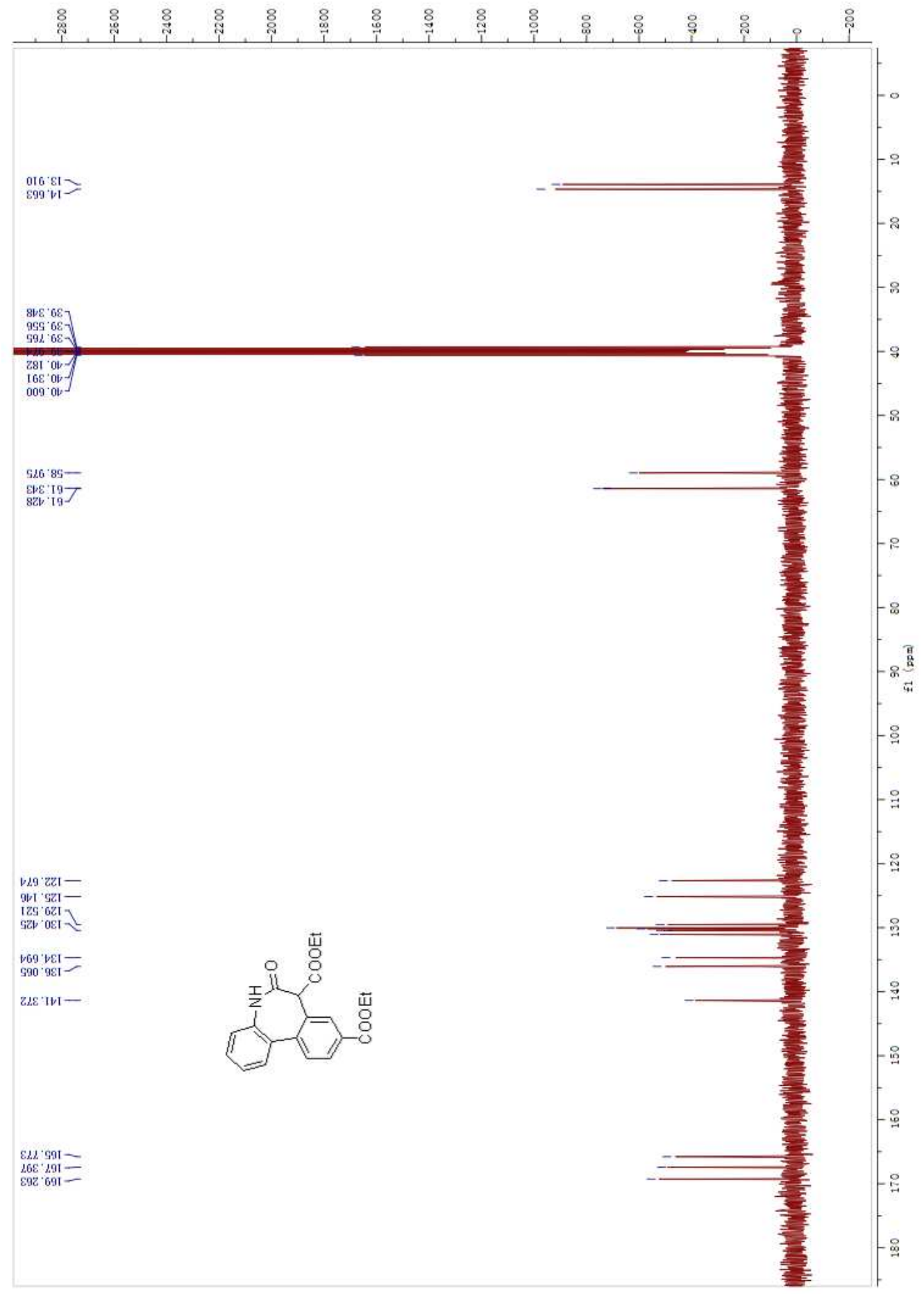


HR-MS (EI) Spectrum of diethyl 6-oxo-6,7-dihydro-5H-dibenzo[b,d]azepine-7,9-di carboxylate $\mathbf{3 f}$

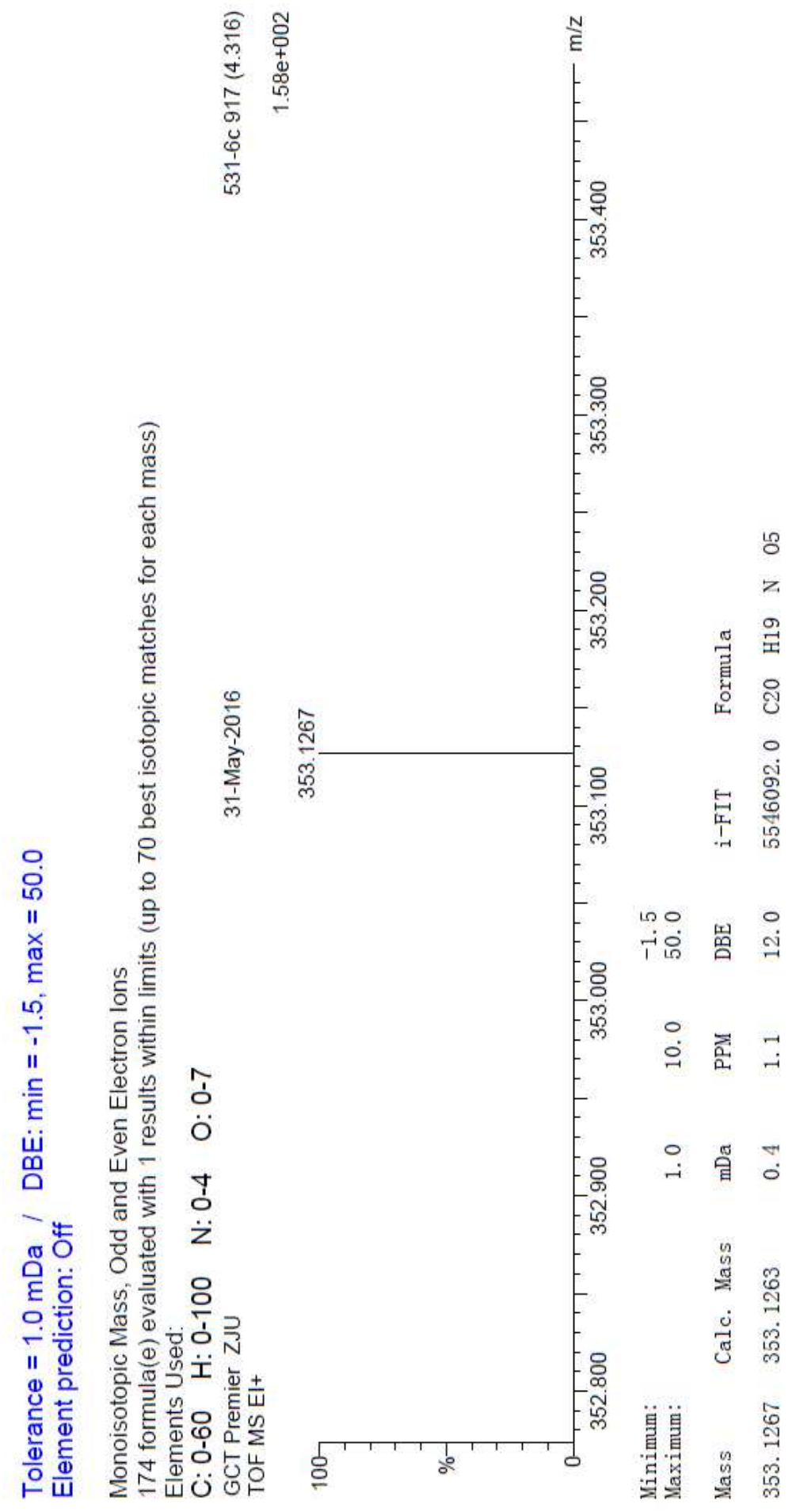


${ }^{1}$ H NMR ethyl 9-nitro-6-oxo-6,7-dihydro-5H-dibenzo[b,d]azepine-7-carboxylate 3g

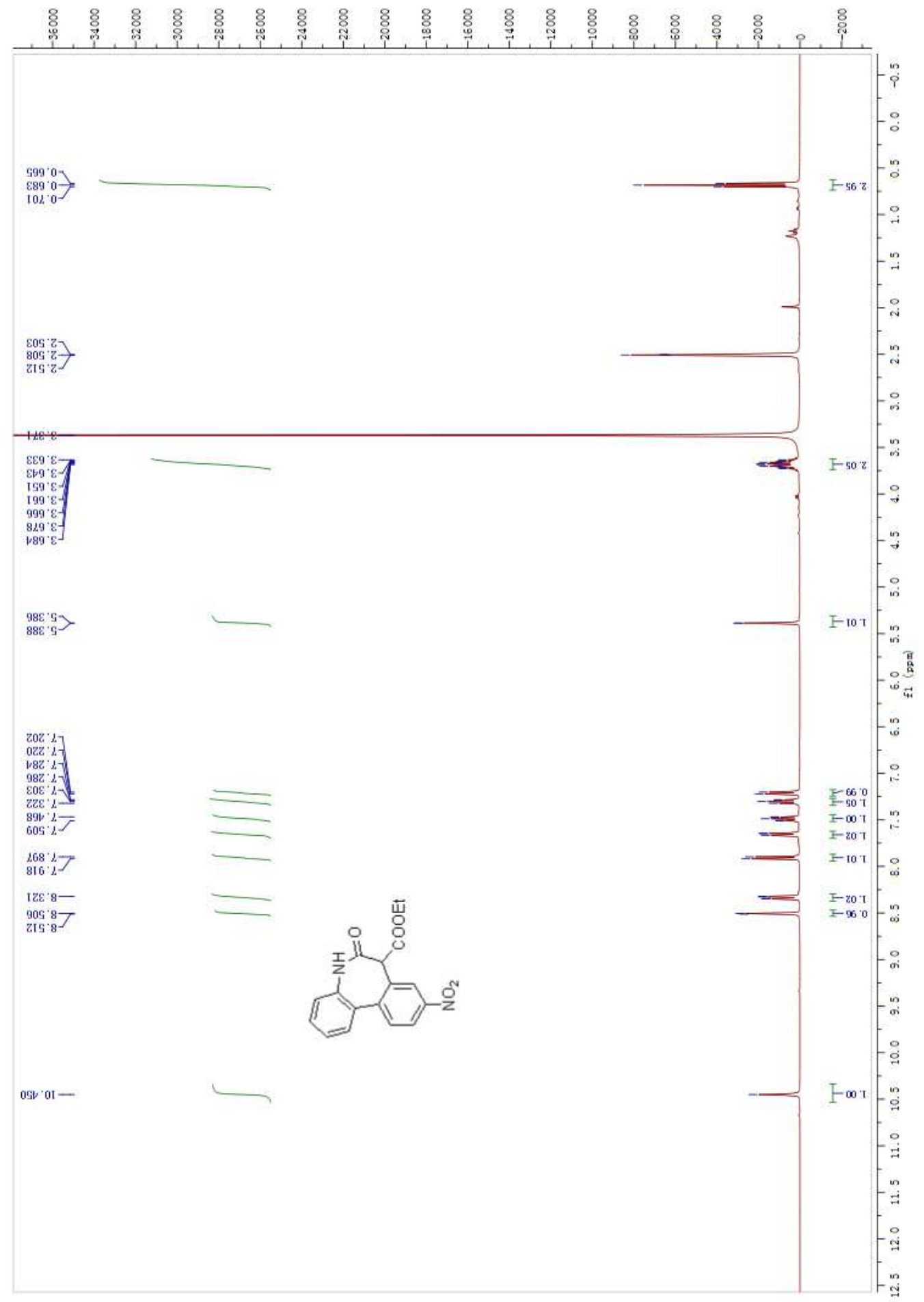


${ }^{13} \mathrm{C}$ NMR ethyl 9-nitro-6-oxo-6,7-dihydro-5H-dibenzo[b,d]azepine-7-carboxylate $3 g$

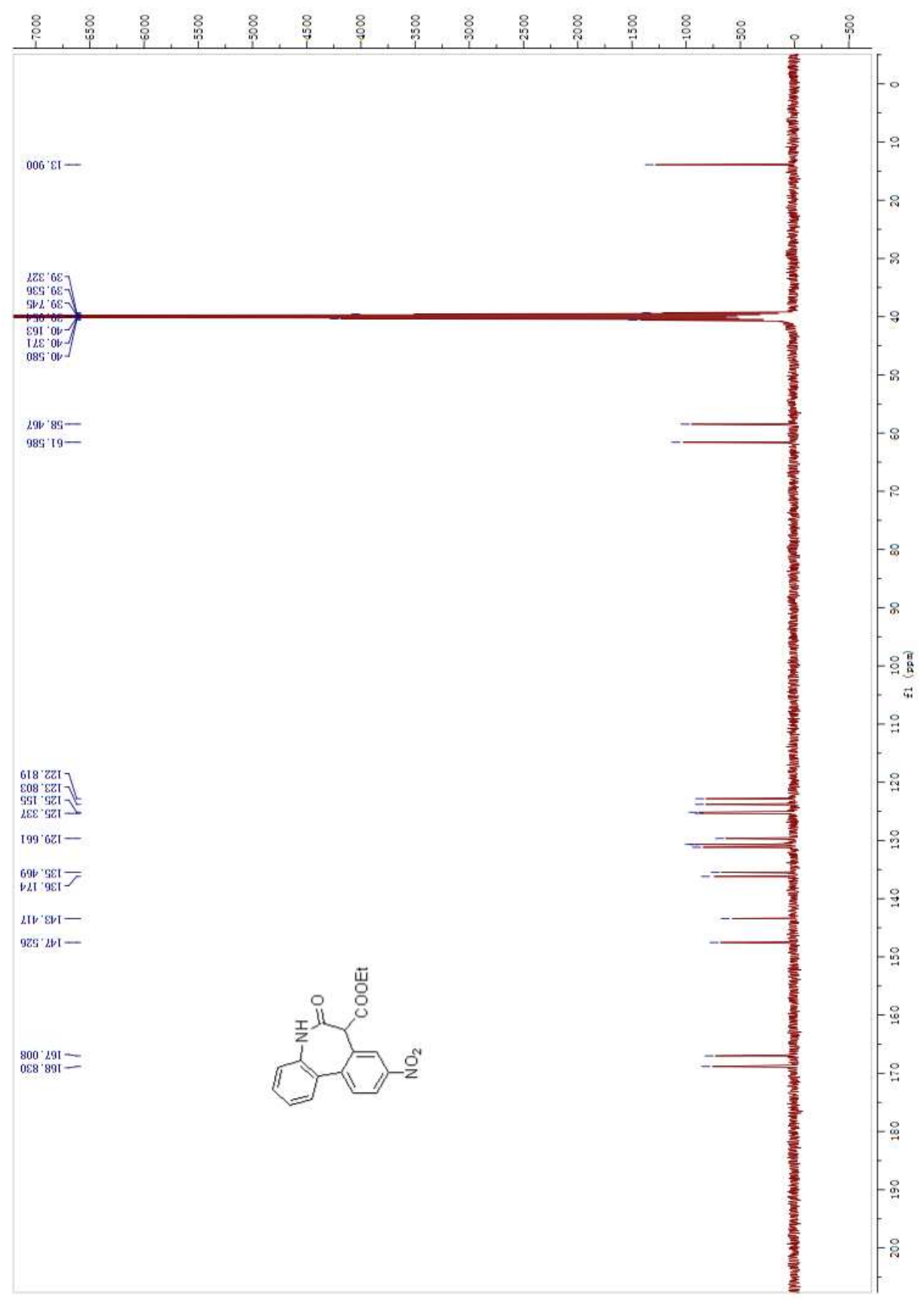


HR-MS (EI) ethyl 9-nitro-6-oxo-6,7-dihydro-5H-dibenzo[b,d]azepine-7-carboxylate 3g

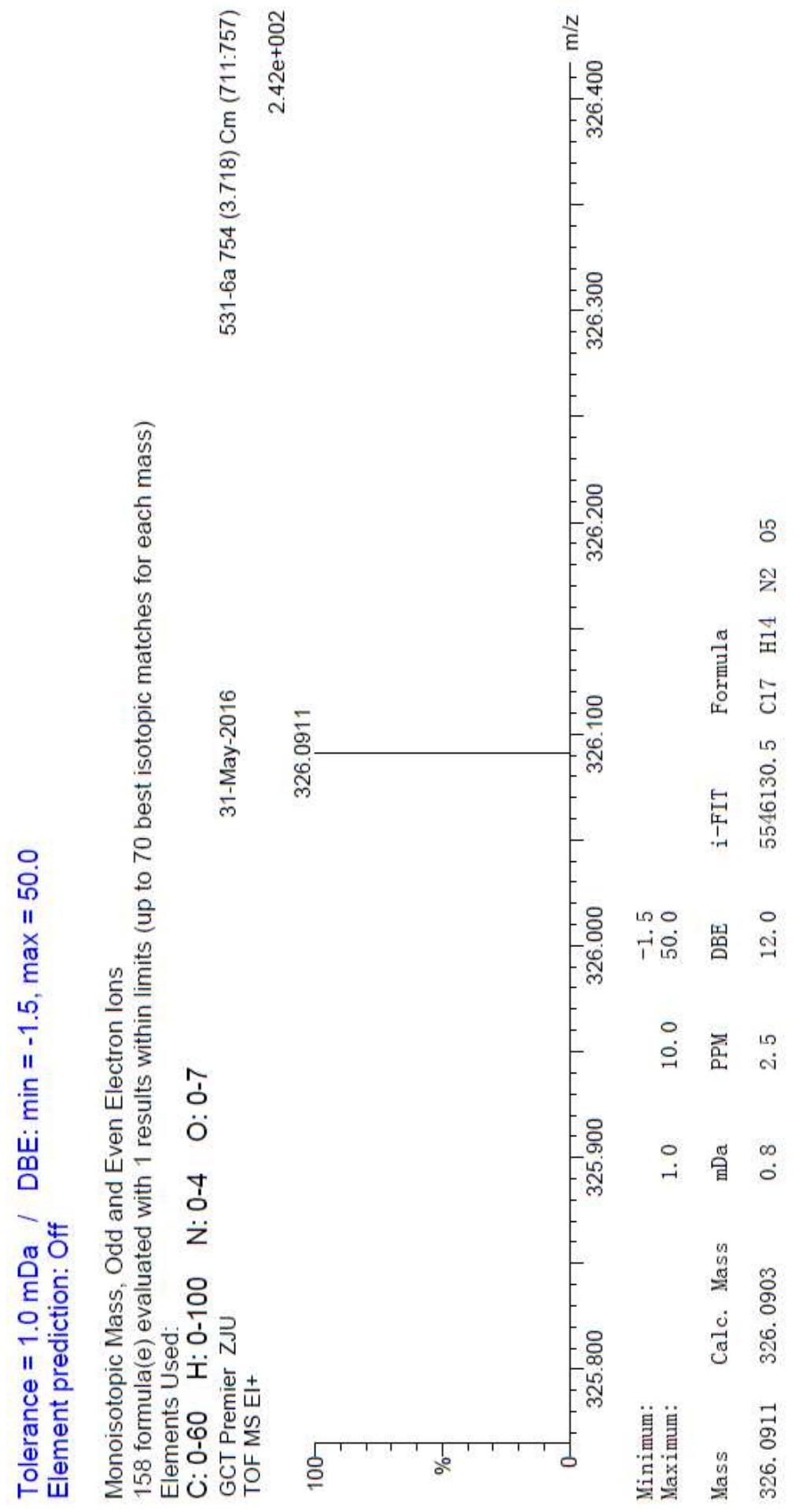


${ }^{1}$ H NMR Spectrum of ethyl 3-methyl-6-oxo-6,7-dihydro-5H-dibenzo[b,d]azepine-7carboxylate $\mathbf{3 h}$

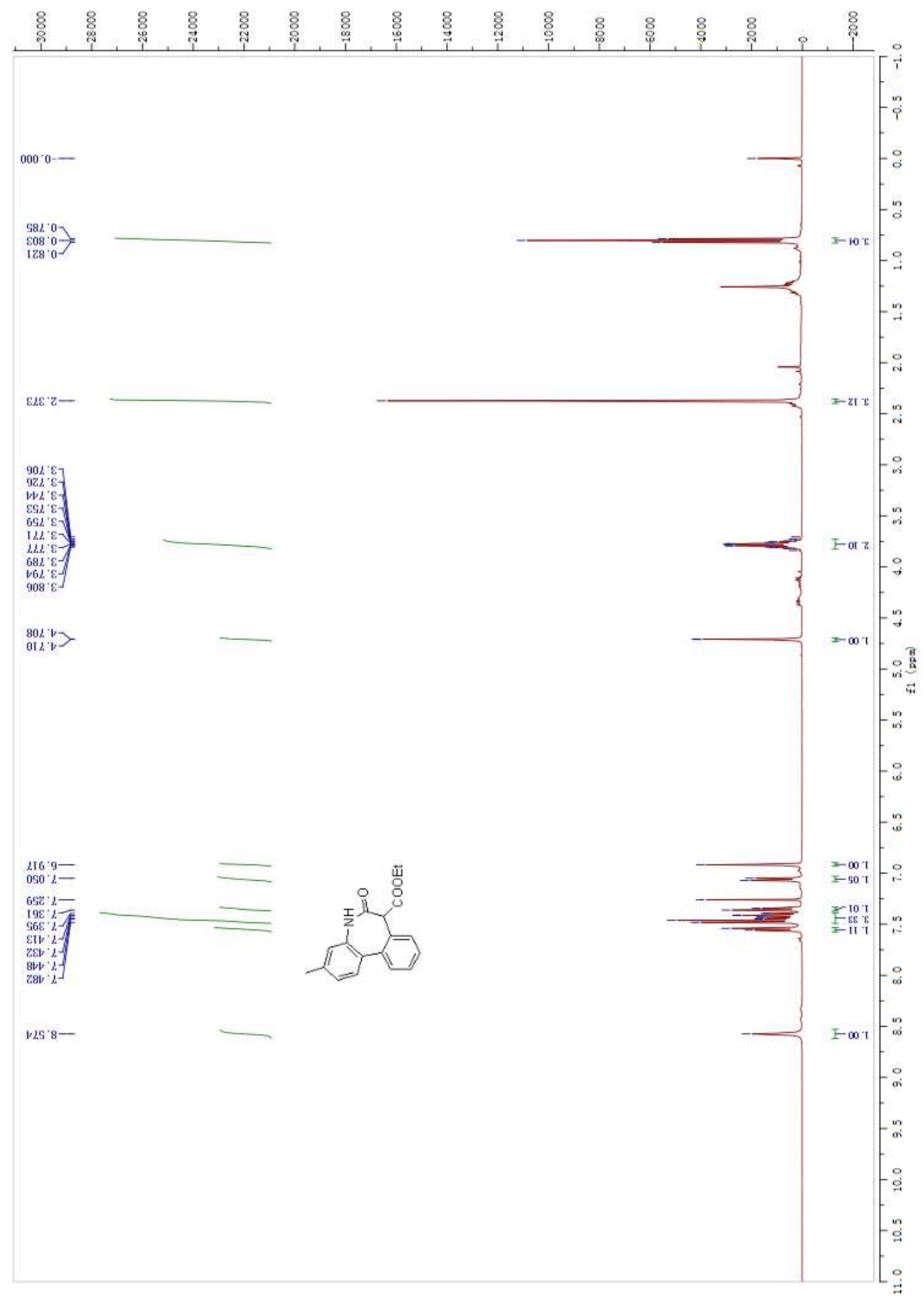


${ }^{13} \mathrm{C}$ NMR Spectrum of ethyl 3-methyl-6-oxo-6,7-dihydro-5H-dibenzo[b,d]azepine-7carboxylate $\mathbf{3 h}$

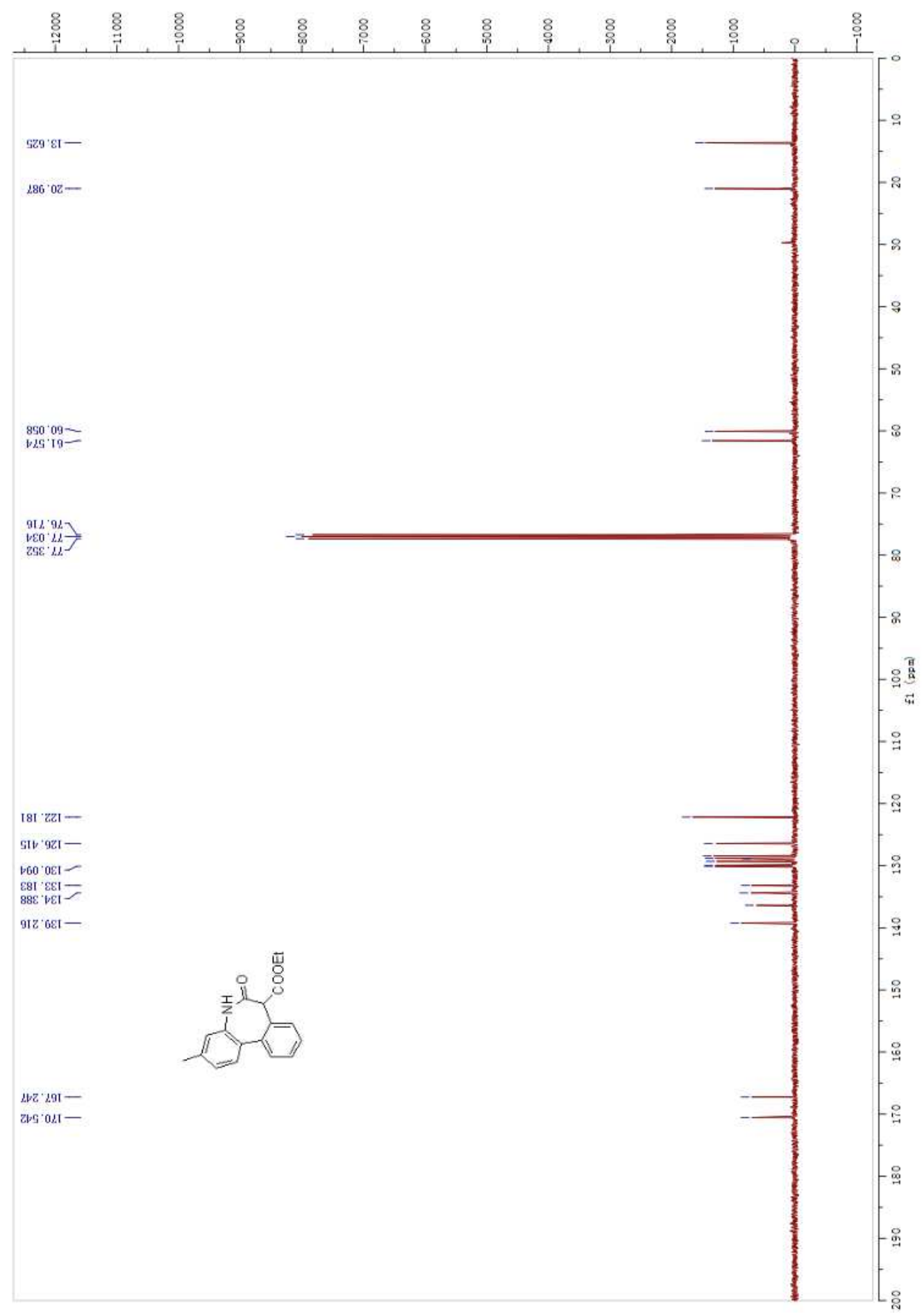


HR-MS (EI) Spectrum of ethyl 3-methyl-6-oxo-6,7-dihydro-5H-dibenzo[b,d]azepine7 - carboxylate $\mathbf{3 h}$

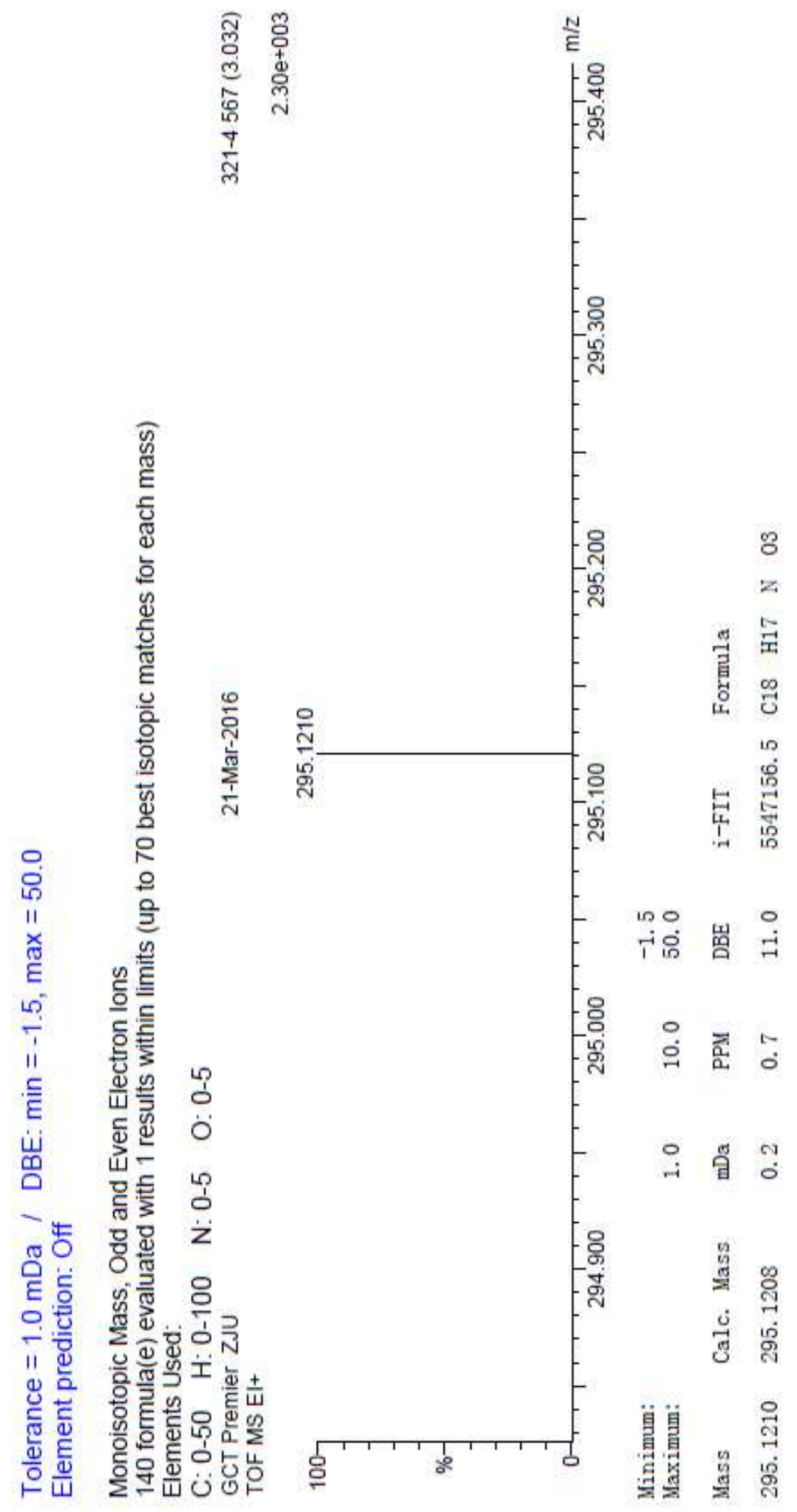


${ }^{1}$ H NMR Spectrum of ethyl 2-methyl-6-oxo-6, 7-dihydro-5H-dibenzo[b,d] azepine -7carboxylate 3i

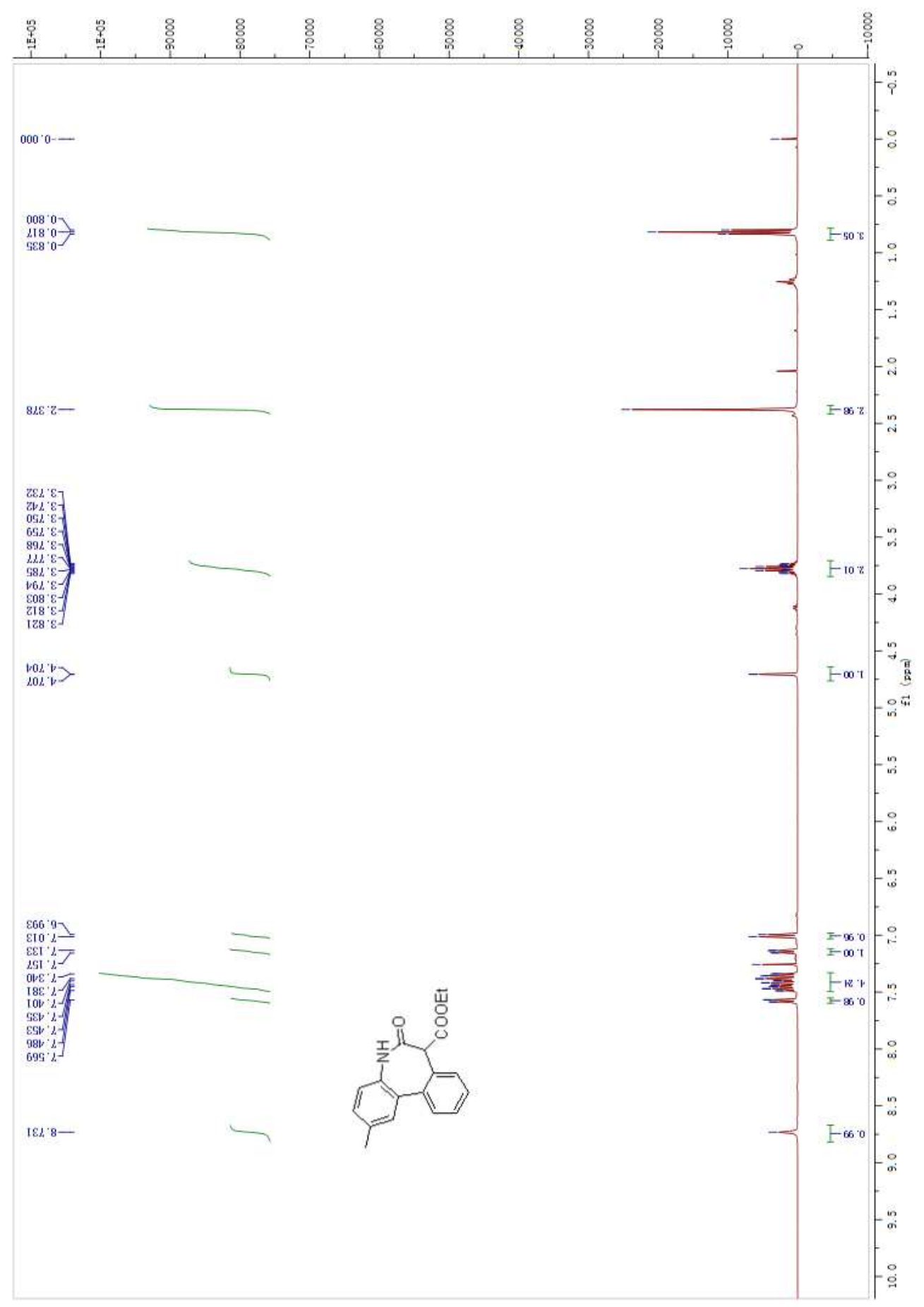


${ }^{13} \mathrm{C}$ NMR Spectrum of ethyl 2-methyl-6-oxo-6, 7-dihydro-5H-dibenzo[b,d] azepine -7- carboxylate $\mathbf{3 i}$

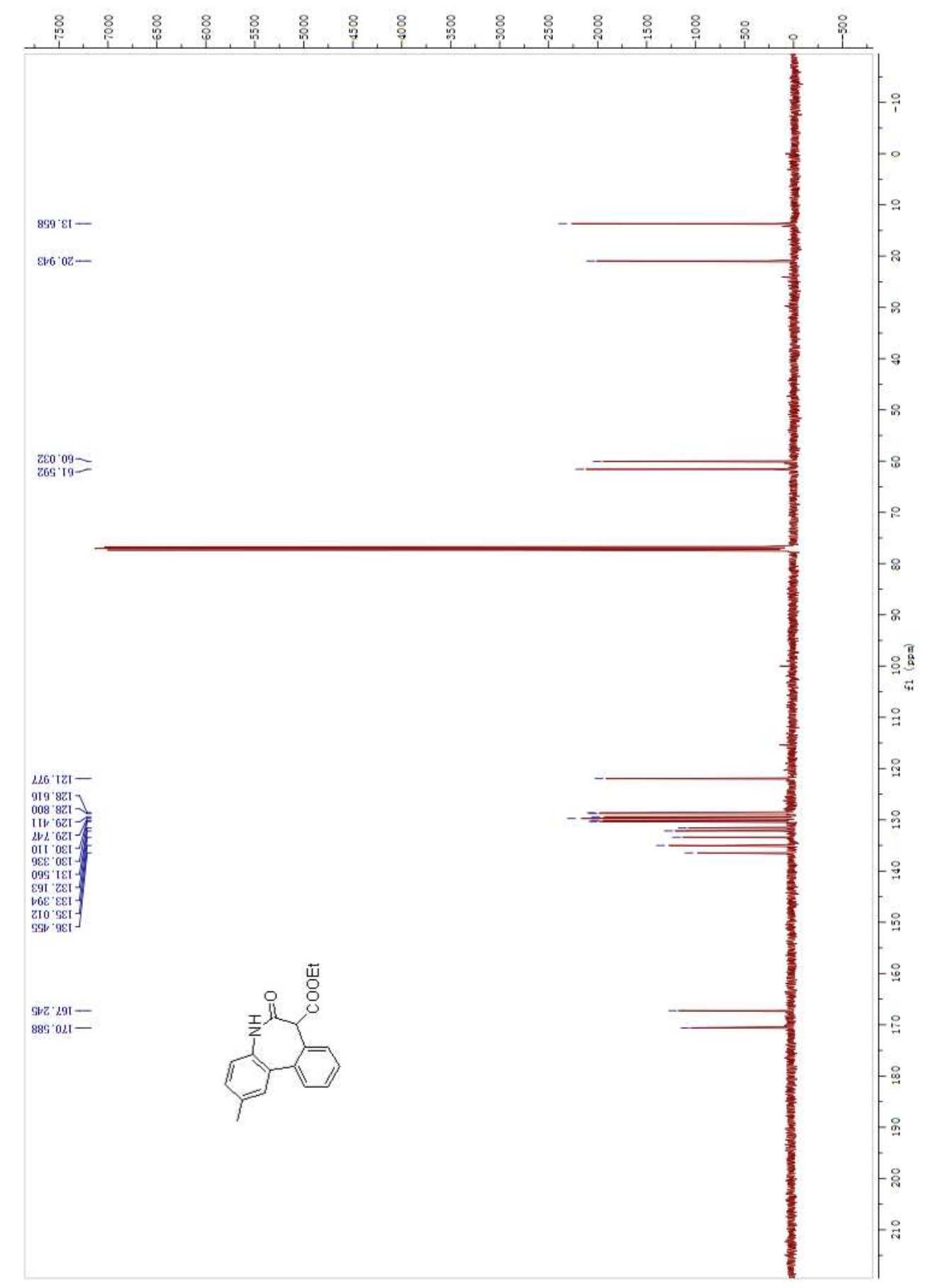


HR-MS (EI) Spectrum of ethyl 2-methyl-6-oxo-6, 7-dihydro-5H-dibenzo[b,d] azepine

-7- carboxylate $\mathbf{3 i}$

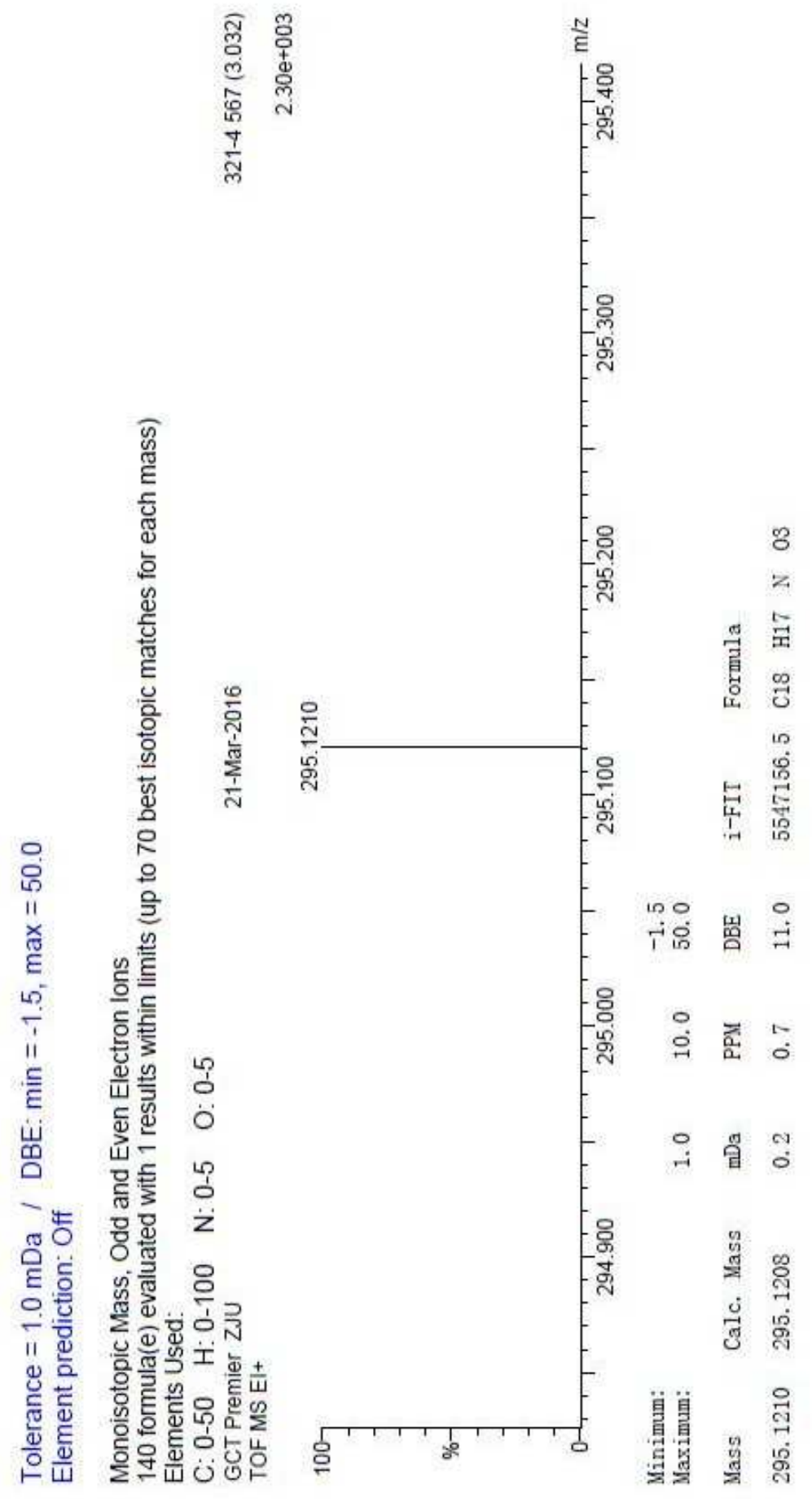


${ }^{1} \mathrm{H}$ NMR Spectrum of ethyl 9-chloro-3-methyl-6-oxo-6,7-dihydro-5H-dibenzo[b,d] azepine-7-carboxylate $\mathbf{3 j}$

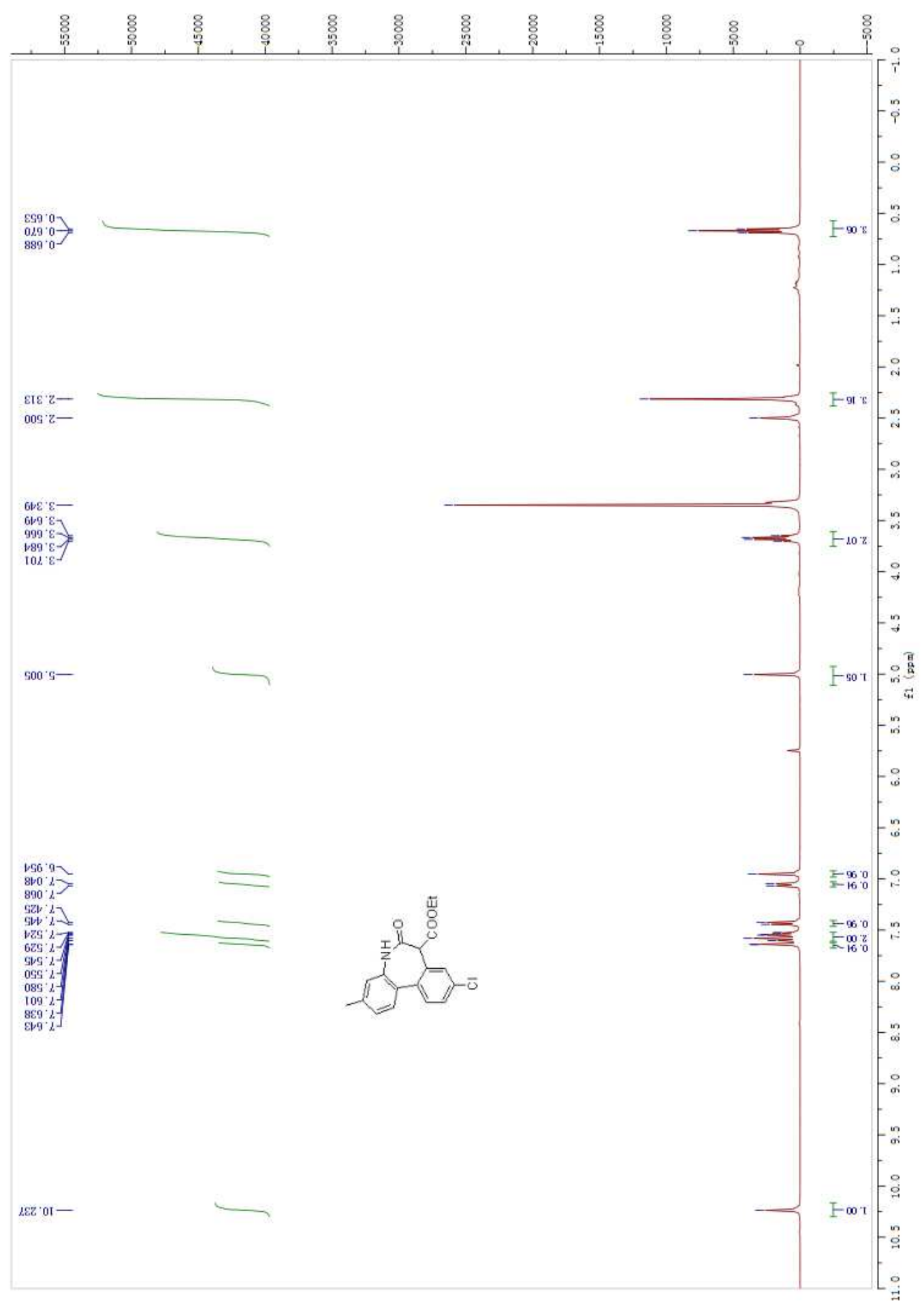


${ }^{13} \mathrm{C}$ NMR Spectrum of ethyl 9-chloro-3-methyl-6-oxo-6,7-dihydro-5H-dibenzo[b,d] azepine-7- carboxylate $\mathbf{3 j}$

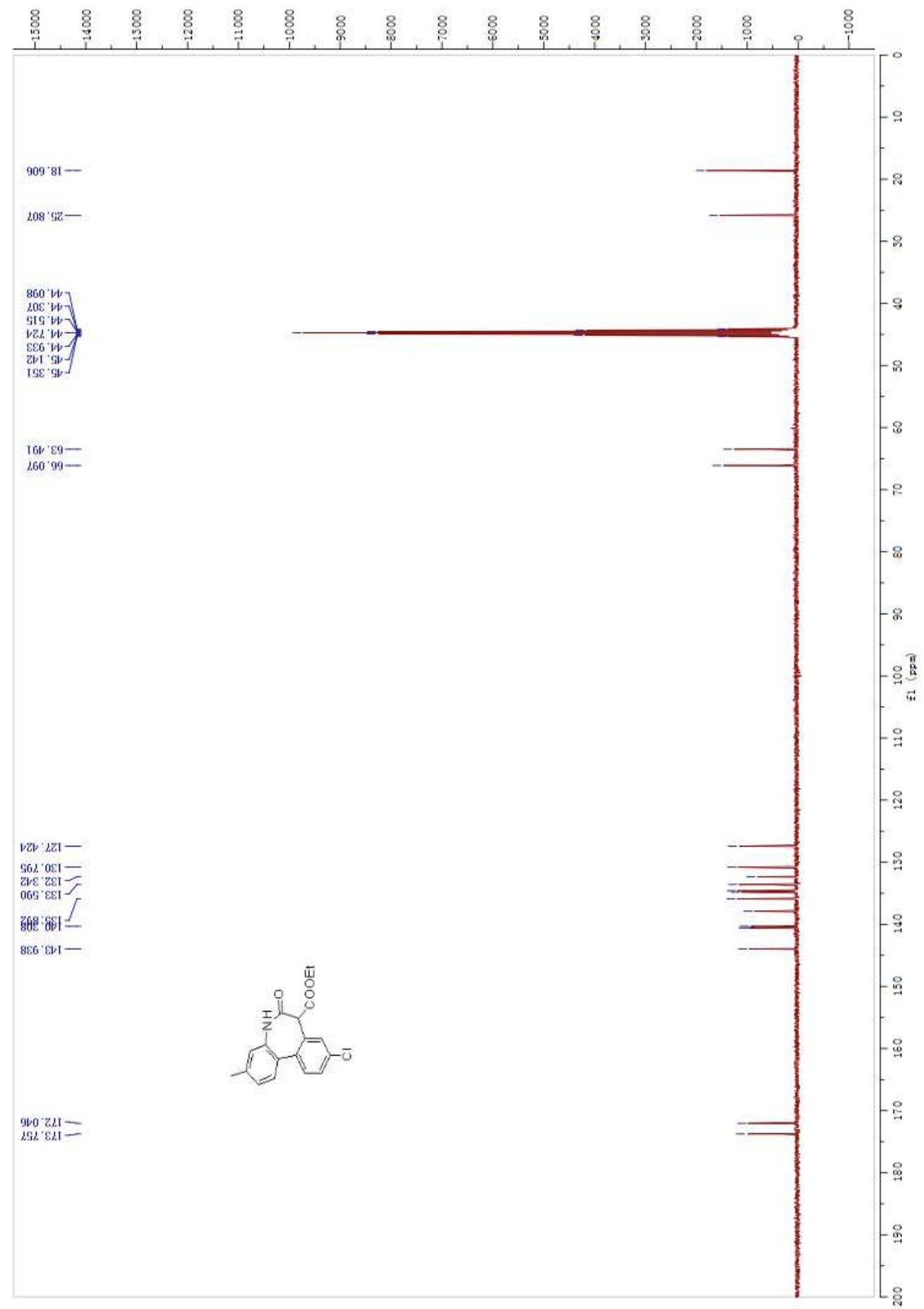


HR-MS (EI) Spectrum of ethyl 9-chloro-3-methyl-6-oxo-6,7-dihydro-5H-dibenzo[b,d] azepine-7- carboxylate $\mathbf{3 j}$

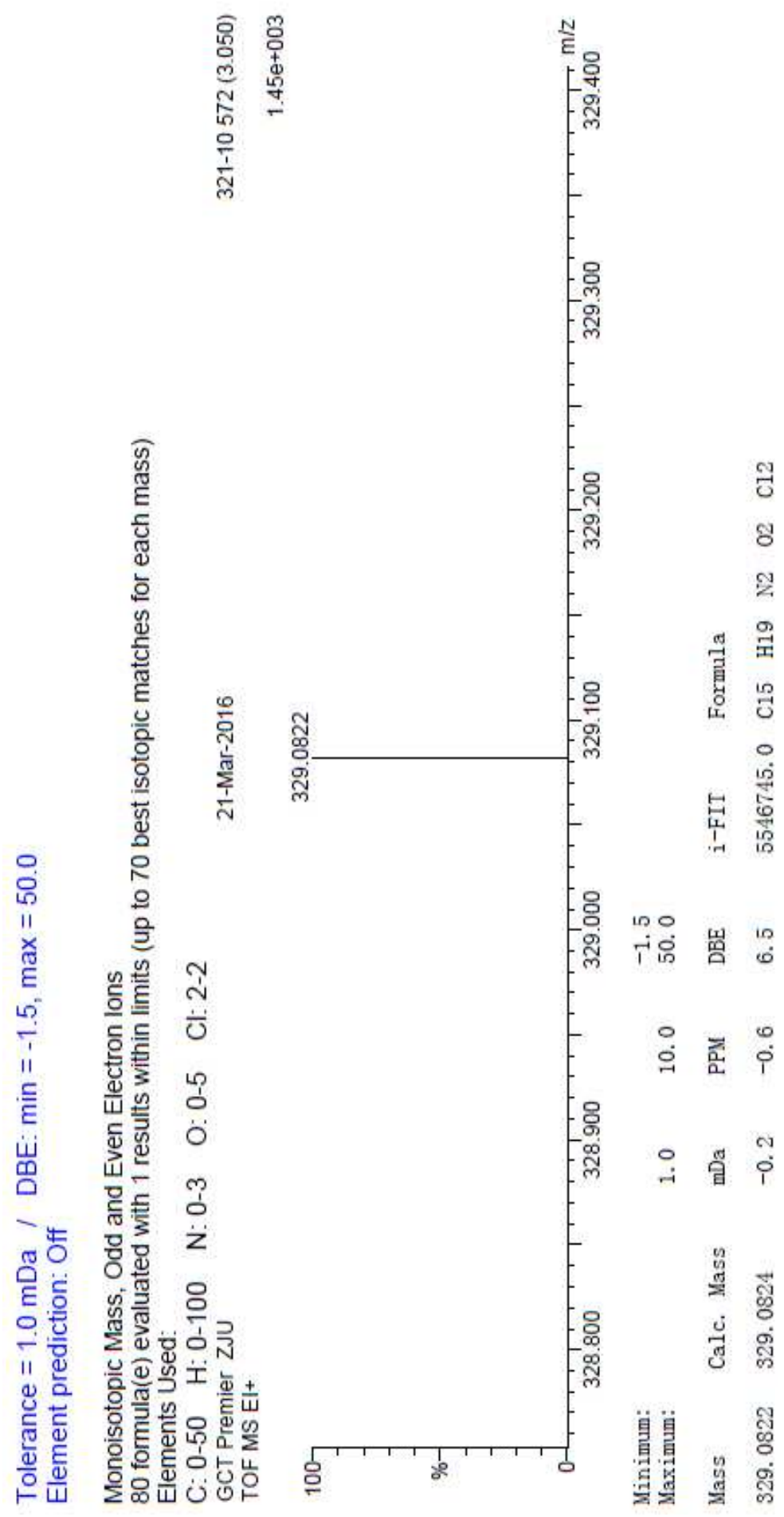


${ }^{1}$ H NMR Spectrum of ethyl 3-fluoro-9-methoxy-6-oxo-6,7-dihydro-5H-dibenzo[b,d] azepine-7- carboxylate $\mathbf{3 k}$

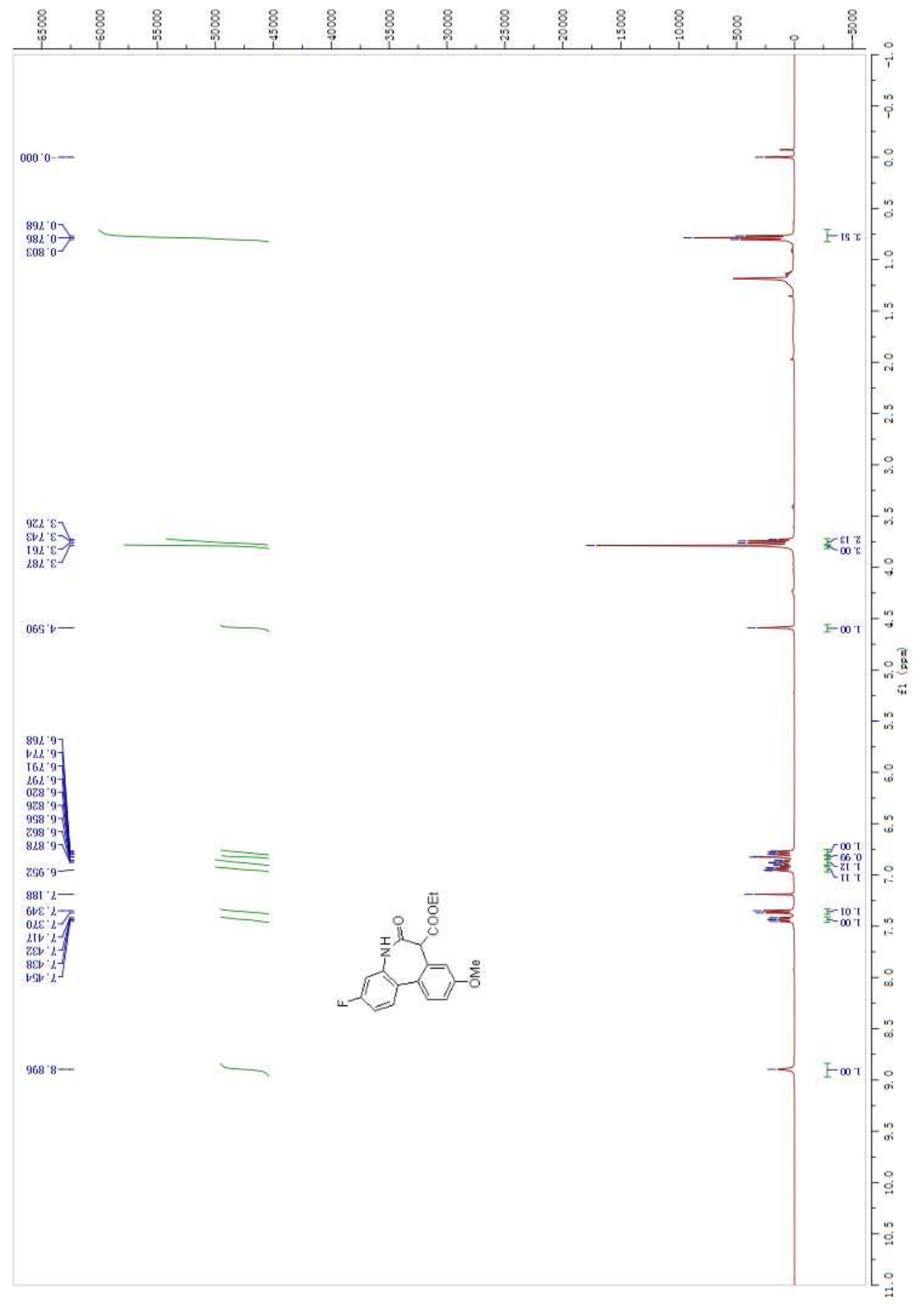


${ }^{13}$ C NMR Spectrum of ethyl 3-fluoro-9-methoxy-6-oxo-6,7-dihydro-5H-dibenzo[b,d] azepine-7- carboxylate $\mathbf{3 k}$

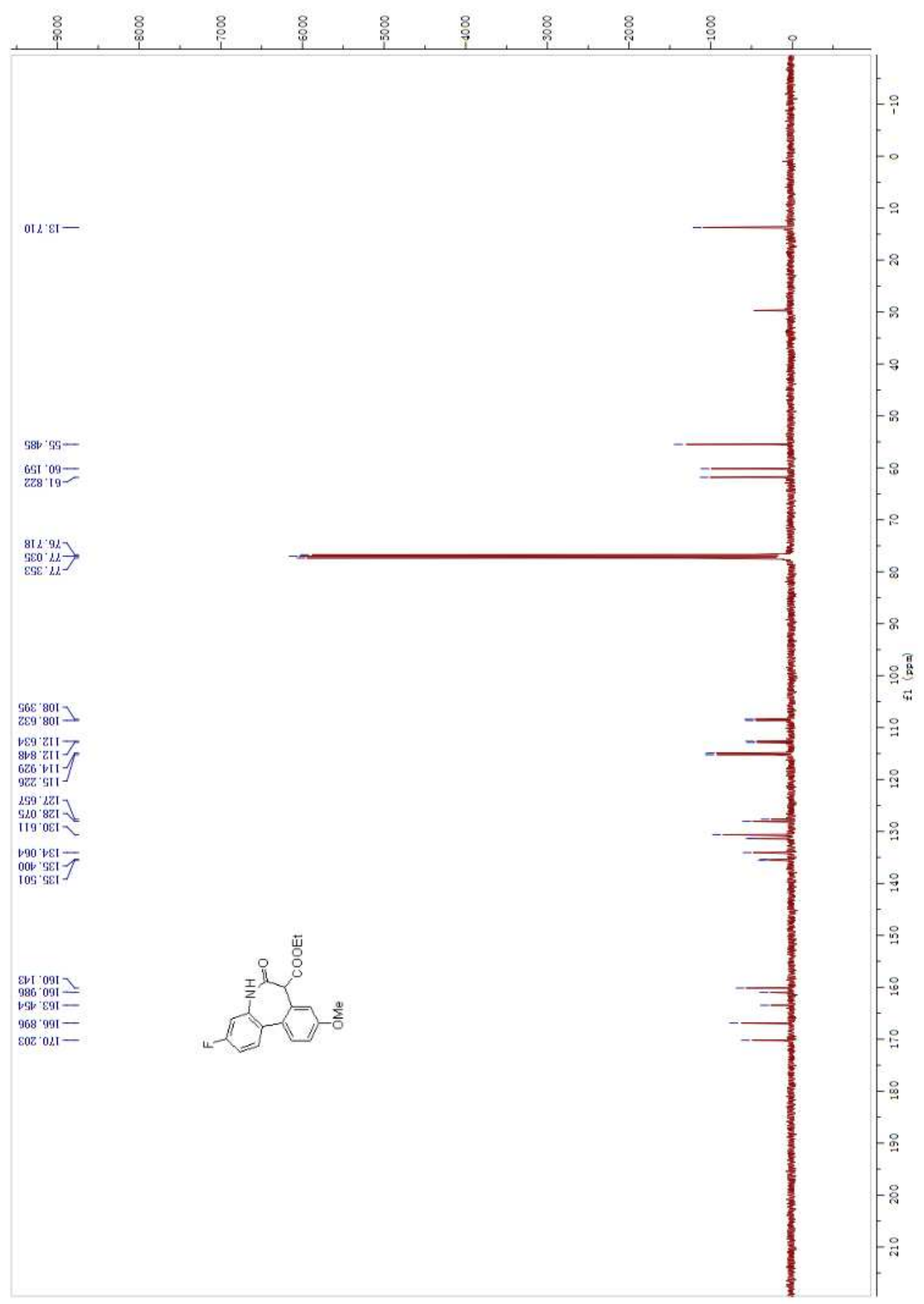


HR-MS (EI) Spectrum of ethyl 3-fluoro-9-methoxy-6-oxo-6,7-dihydro-5H-dibenzo [b,d] azepine -7- carboxylate $\mathbf{3 k}$

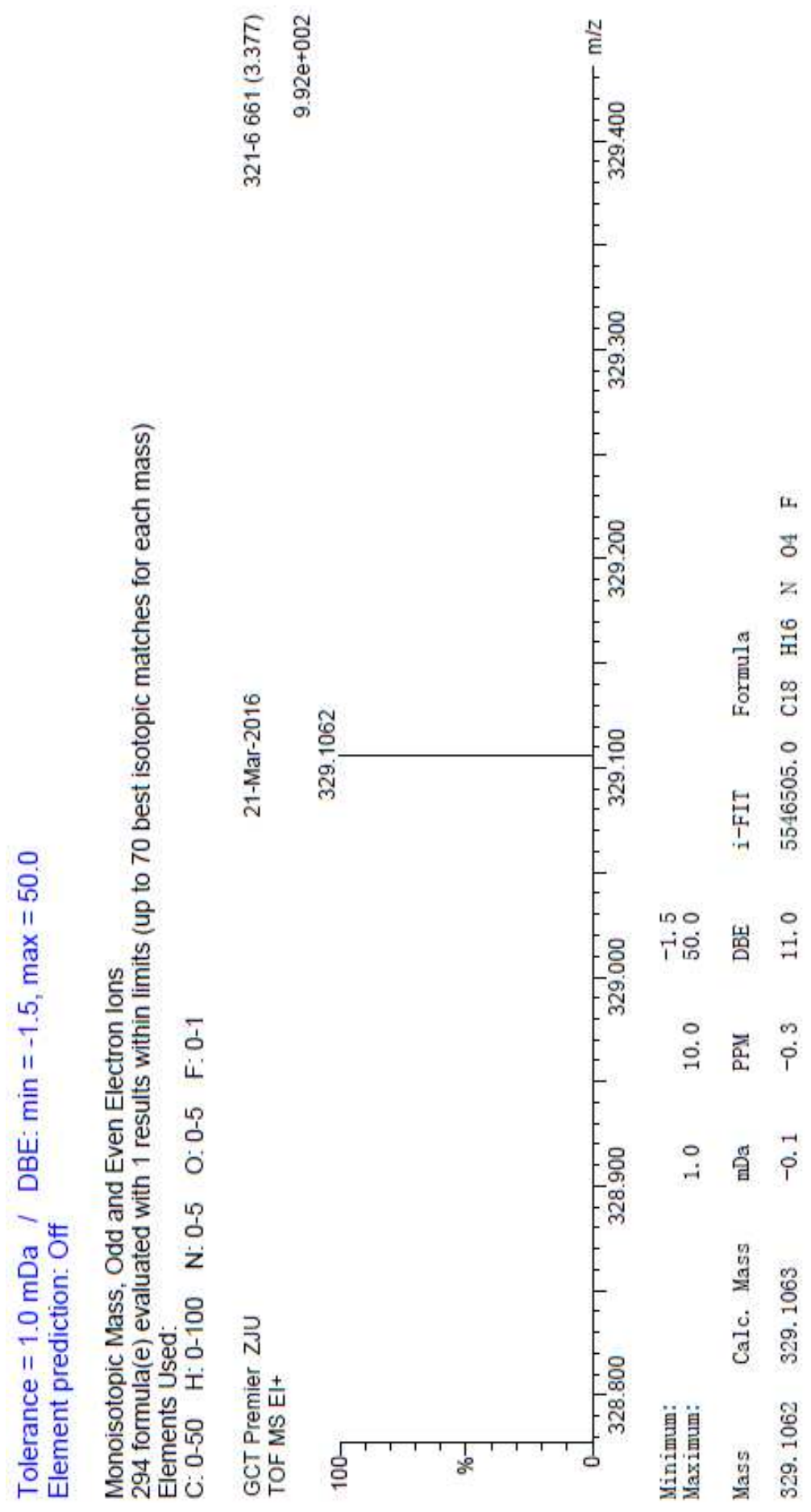


${ }^{1}$ H NMR Spectrum of ethyl 9-methoxy-3-methyl-6-oxo-6,7-dihydro-5H-dibenzo [b,d] azpin e -7-carboxylate $\mathbf{3 l}$

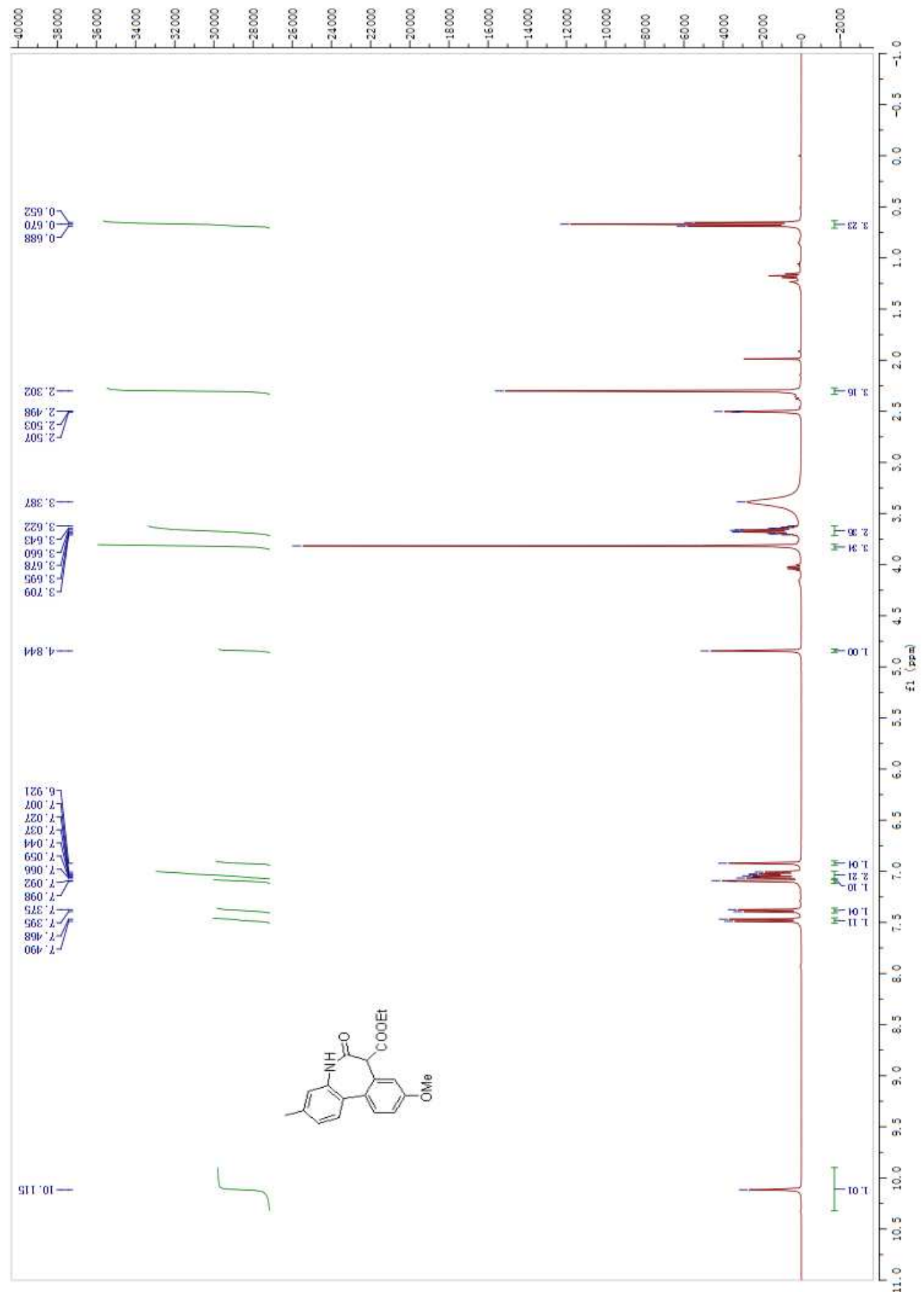


${ }^{13} \mathrm{C}$ NMR Spectrum of ethyl 9-methoxy-3-methyl-6-oxo-6,7-dihydro-5H-dibenzo[b,d] azepine -7-carboxylate $\mathbf{3 l}$

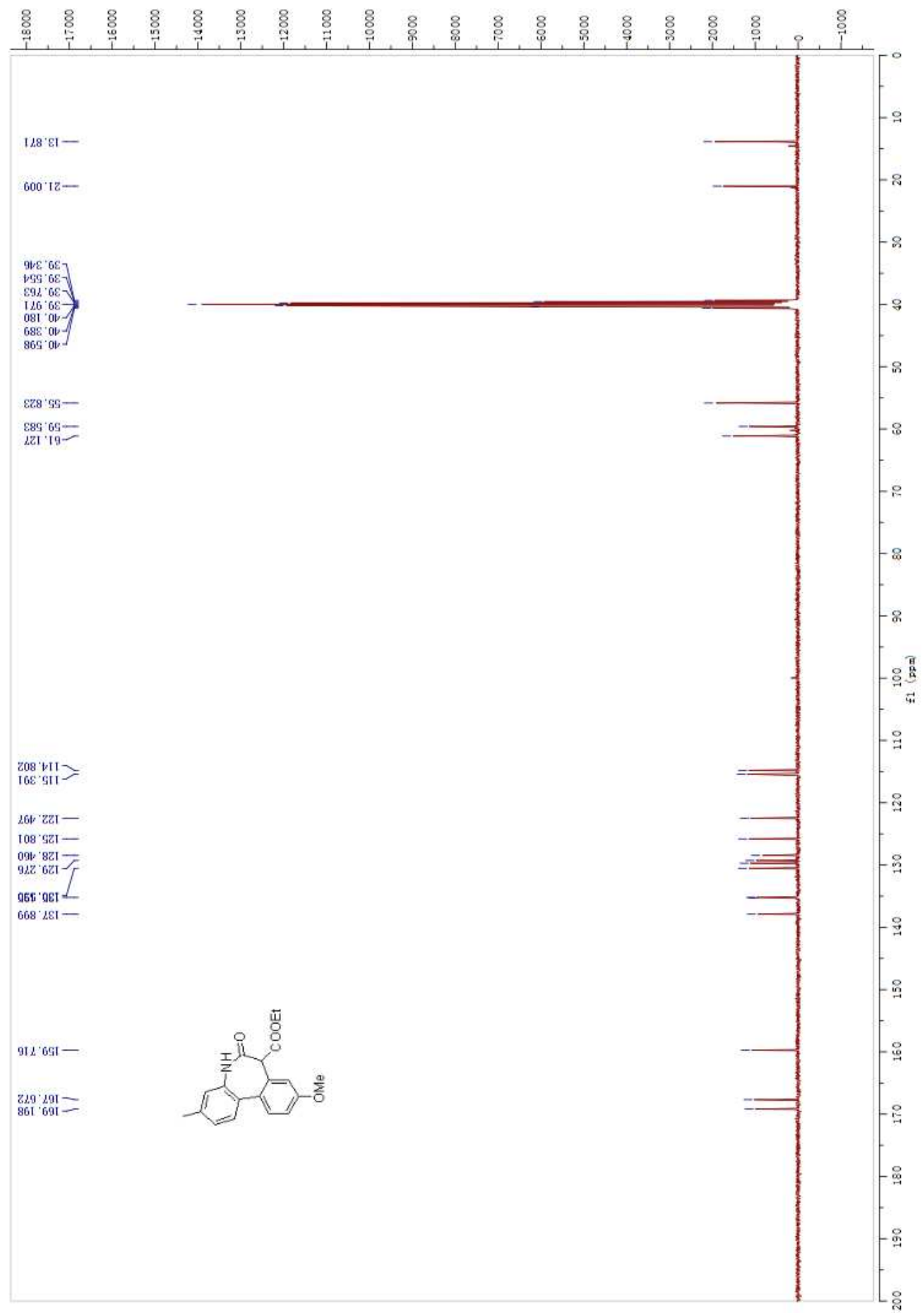


HR-MS (EI) Spectrum of ethyl 9-methoxy-3-methyl-6-oxo-6,7-dihydro-5H-dibenzo [b,d]azepine - 7- carboxylate 31

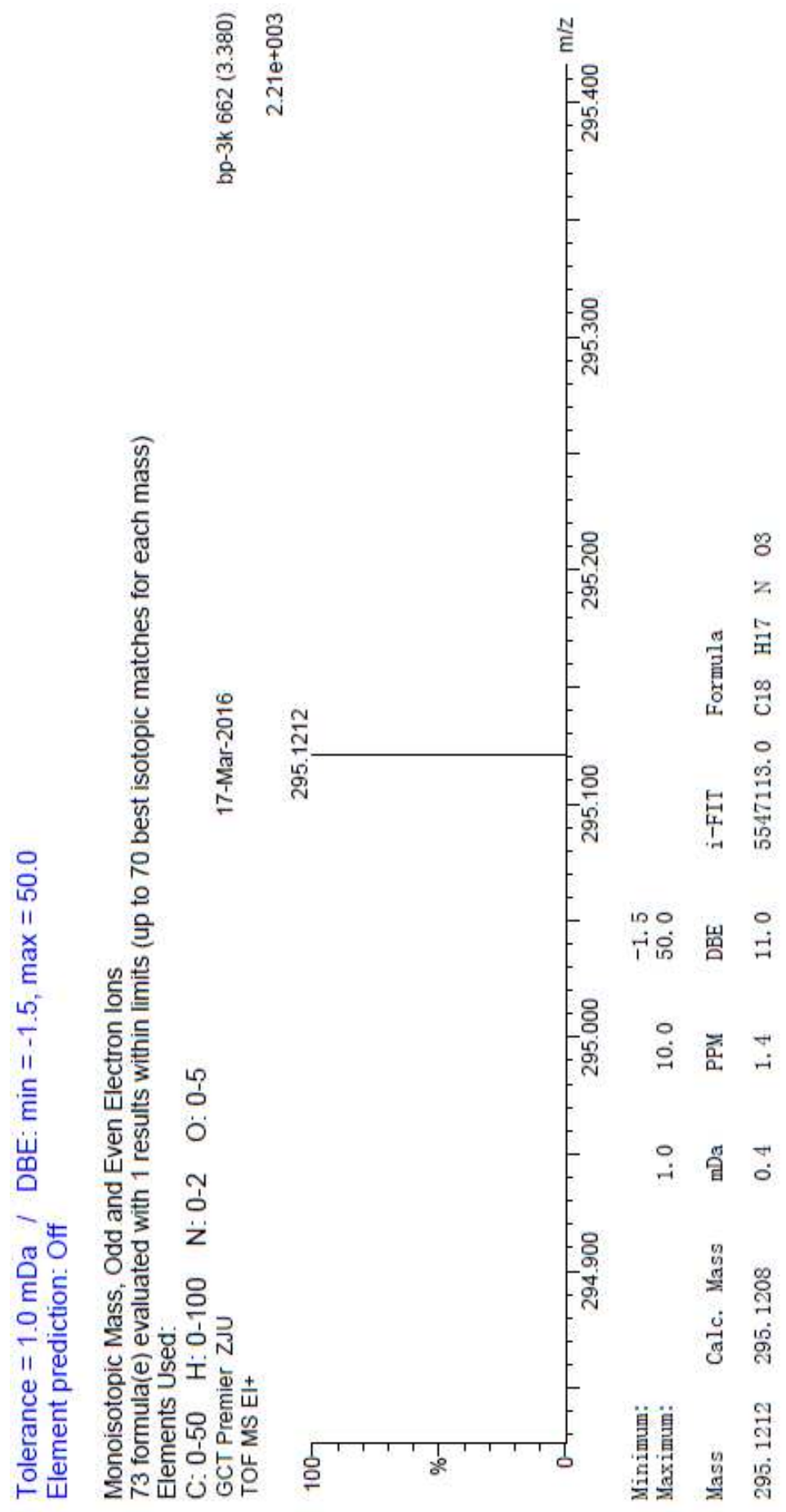


${ }^{1} \mathrm{H}$ NMR Spectrum of ethyl 9-chloro-2-methyl-6-oxo-6,7-dihydro-5H-dibenzo[b,d] azepine-7- carboxylate $\mathbf{3 m}$

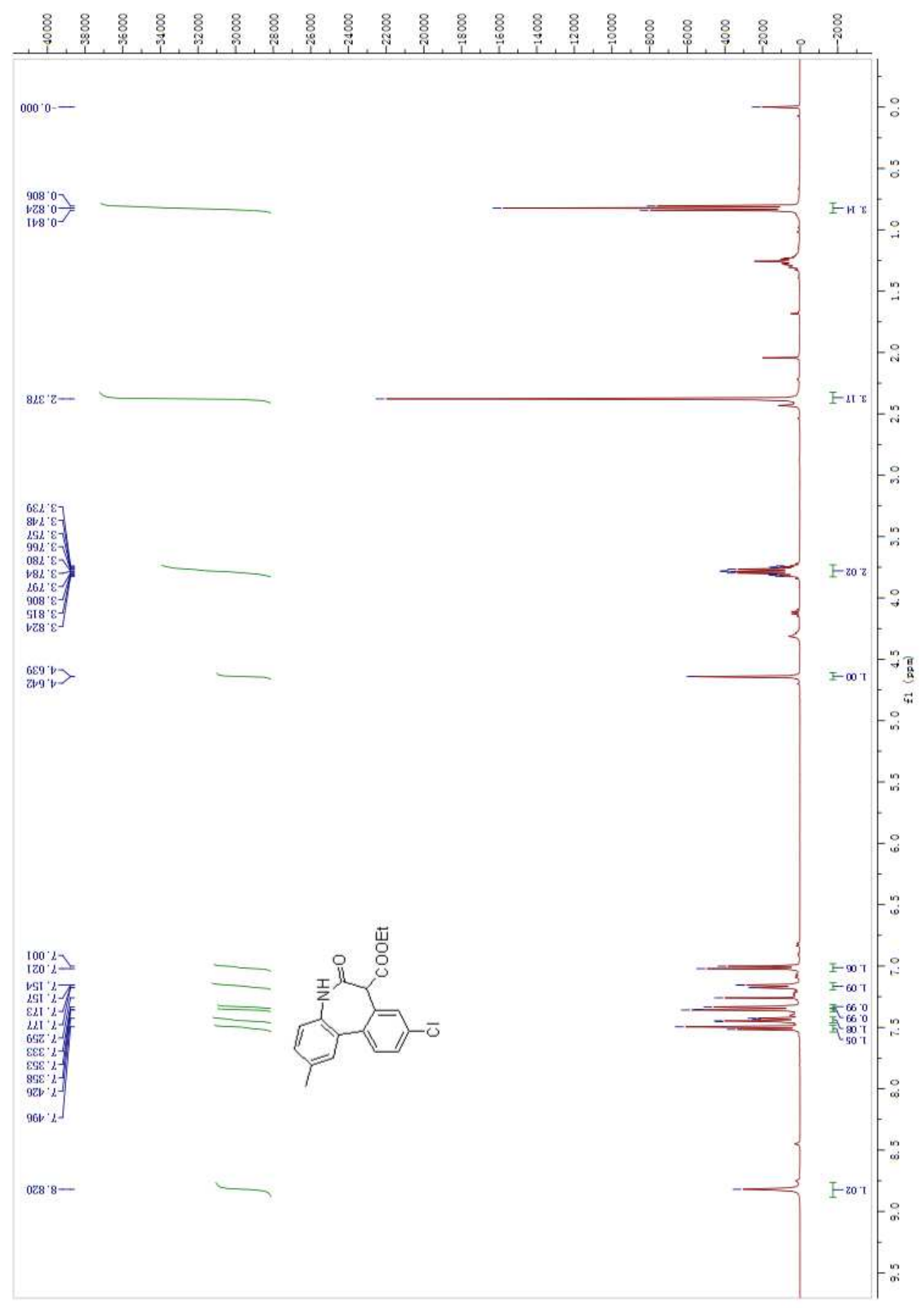


${ }^{13} \mathrm{C}$ NMR Spectrum of ethyl 9-chloro-2-methyl-6-oxo-6,7-dihydro-5H-dibenzo[b,d] azepine-7- carboxylate $\mathbf{3 m}$

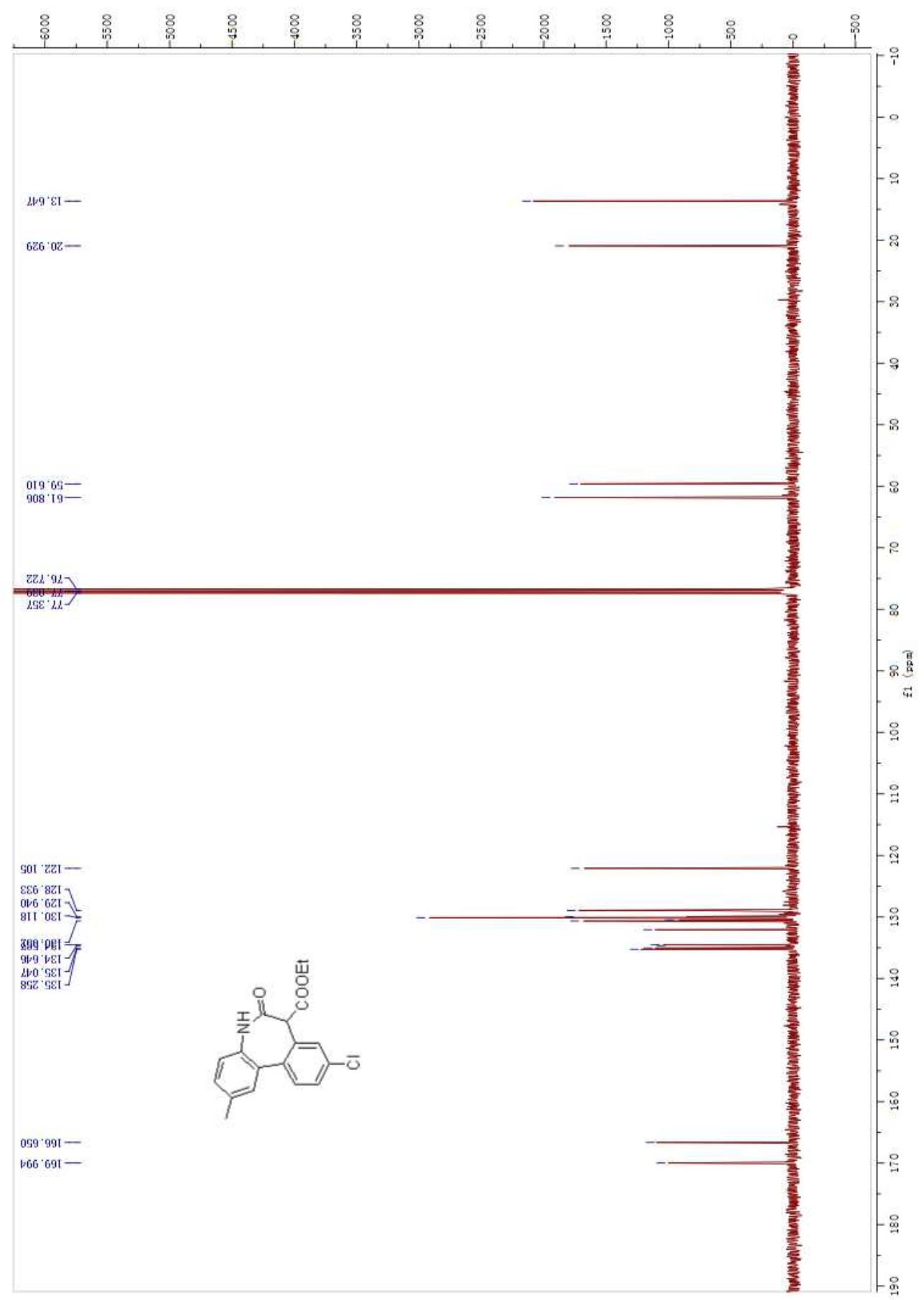


HR-MS (EI) Spectrum of ethyl 9-chloro-2-methyl-6-oxo-6,7-dihydro-5H-dibenzo[b,d] azepine -7- carboxylate $\mathbf{3 m}$

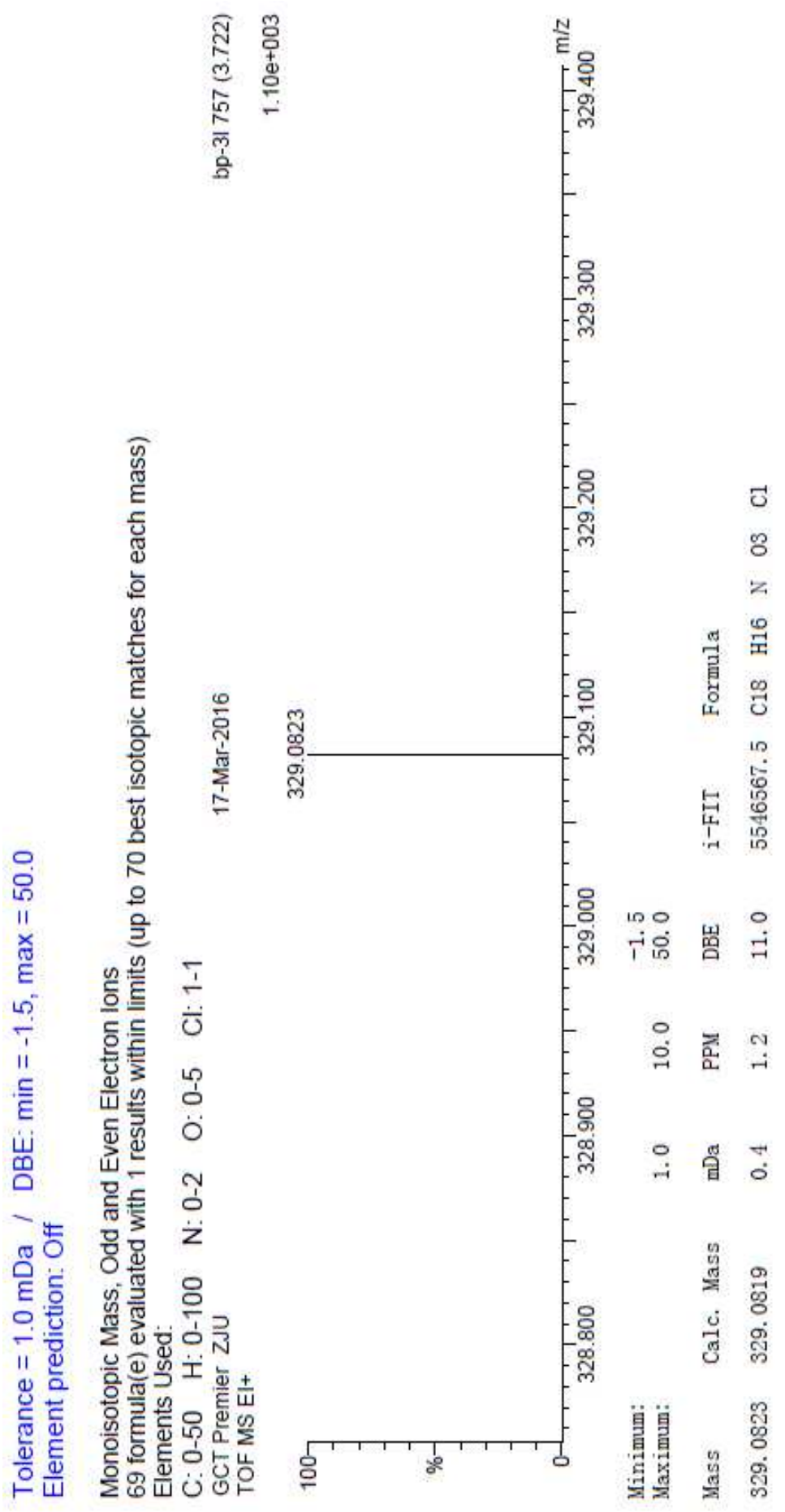


${ }^{1}$ H NMR of Spectrum ethyl 6-oxo-6,7-dihydro-5H-benzo[b]naphtho[2,3-d]azepine-7carboxylate 3n

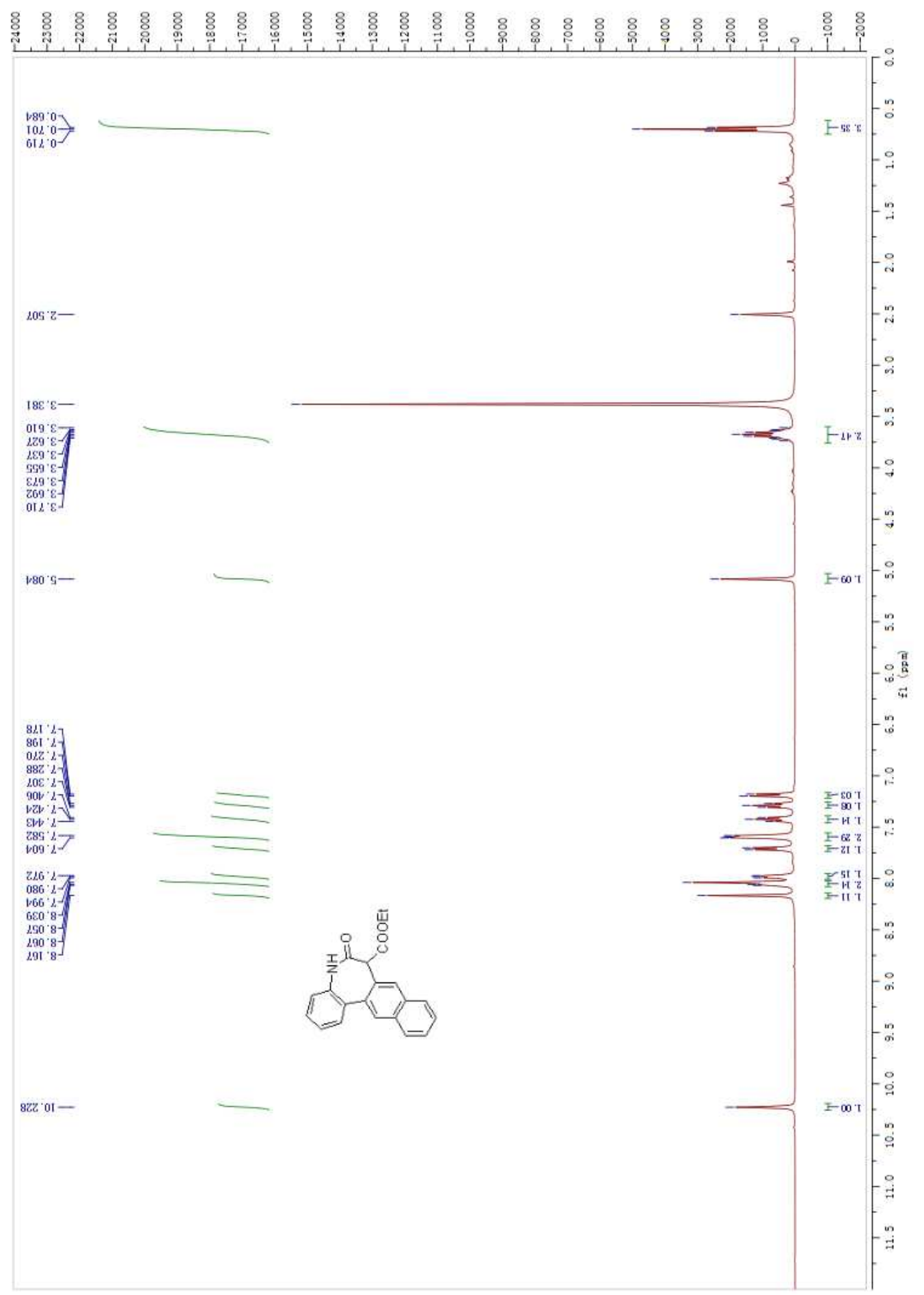


${ }^{13}$ C NMR Spectrum of ethyl 6-oxo-6,7-dihydro-5H-benzo[b]naphtho[2,3-d]azepine-7carboxylate 3n

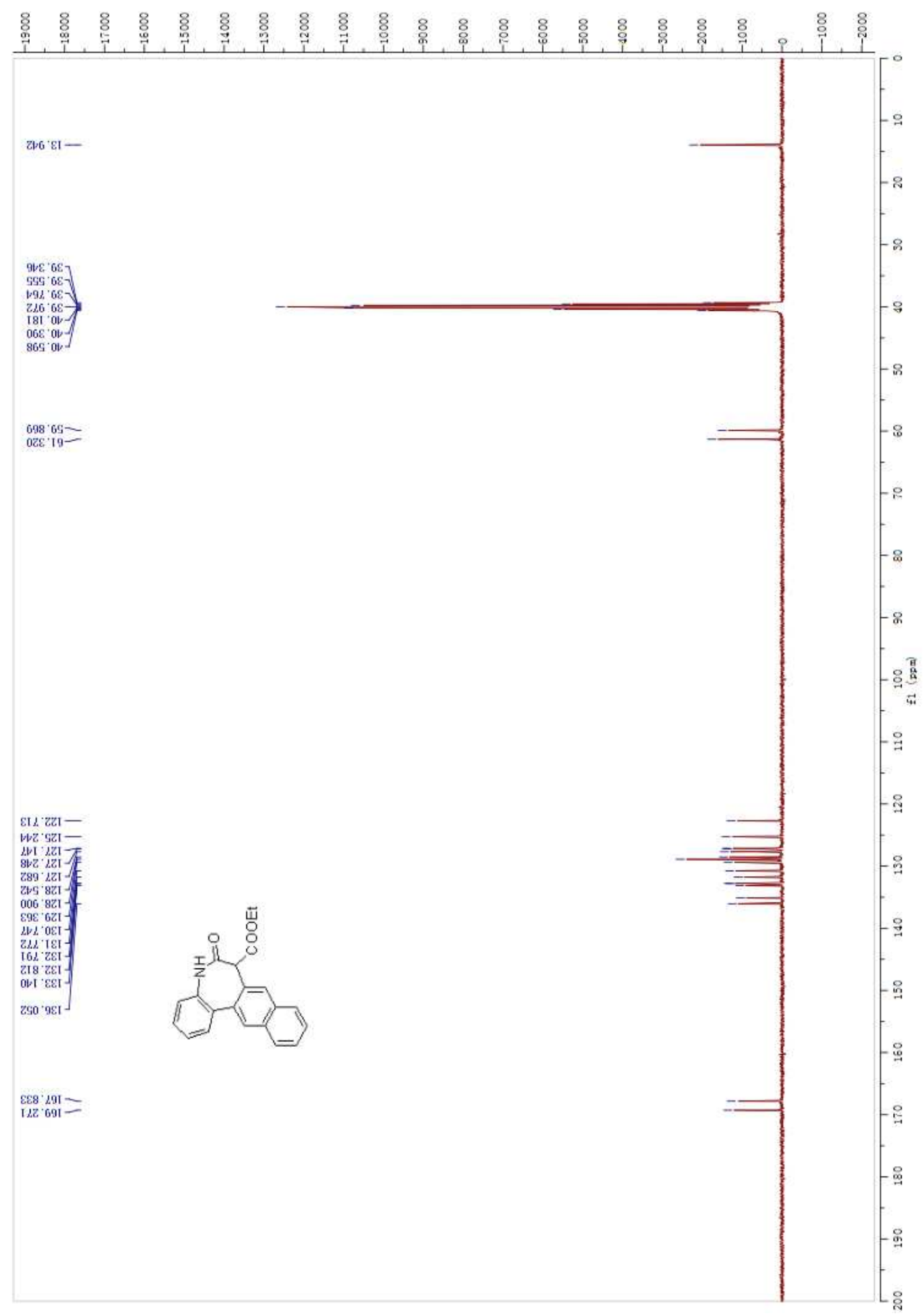


HR-MS (EI) Spectrum of ethyl 6-oxo-6,7-dihydro-5H-benzo[b]naphtho[2,3-d]azepine -7- carboxylate $3 \mathbf{n}$

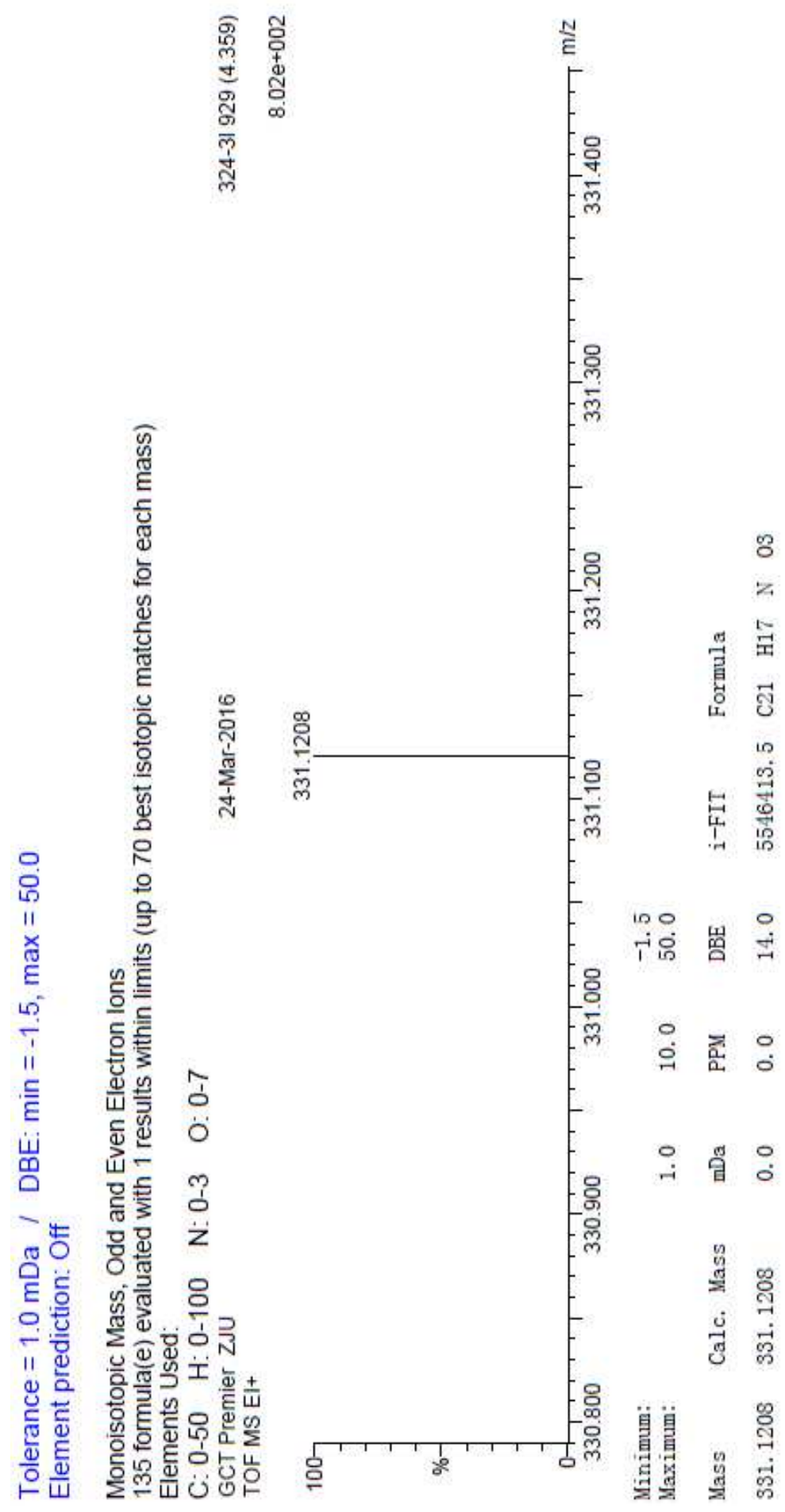


${ }^{1} \mathrm{H}$ NMR Spectrum of ethyl 2-methyl-6-oxo-6,7-dihydro-5H-benzo[b]naphtho[2,3-d] azepine-7- carboxylate 30

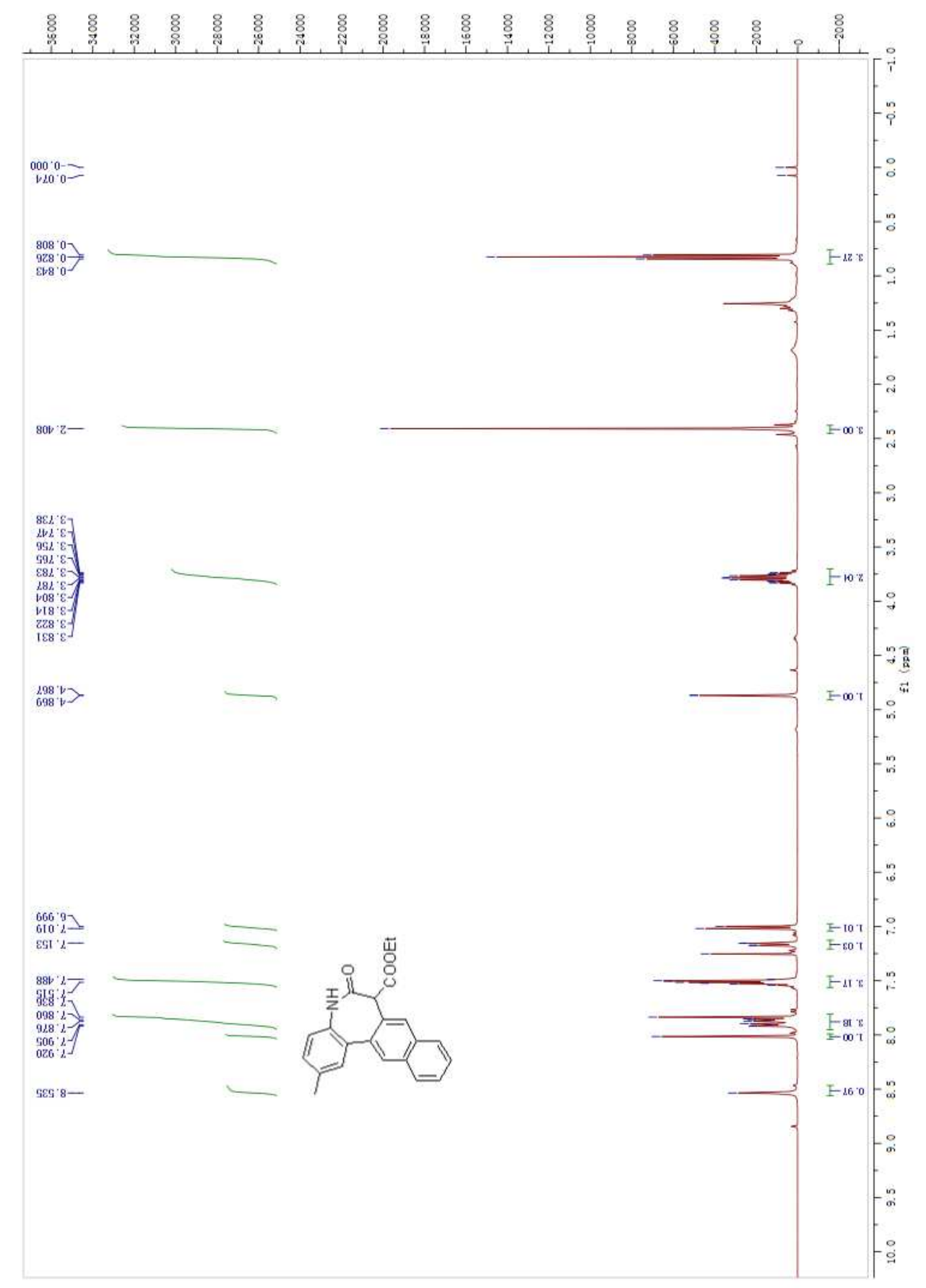


${ }^{13} \mathrm{C}$ NMR Spectrum of ethyl 2-methyl-6-oxo-6,7-dihydro-5H-benzo[b]naphtho[2,3-d] azepine-7- carboxylate 30

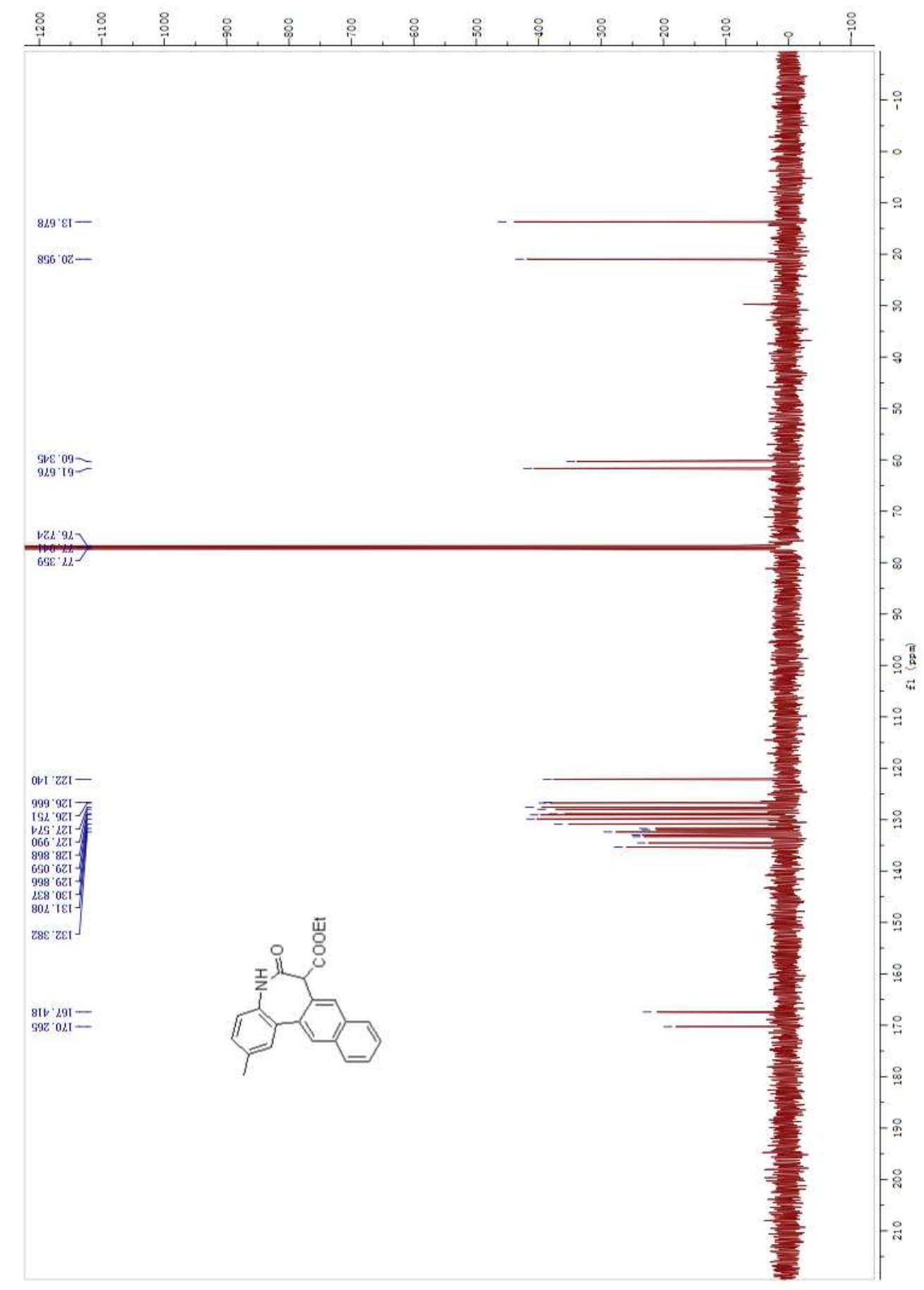


HR-MS (EI) Spectrum of ethyl 2-methyl-6-oxo-6,7-dihydro-5H-benzo[b]naphtha [2,3-d]azepine - 7 - carboxylate 30

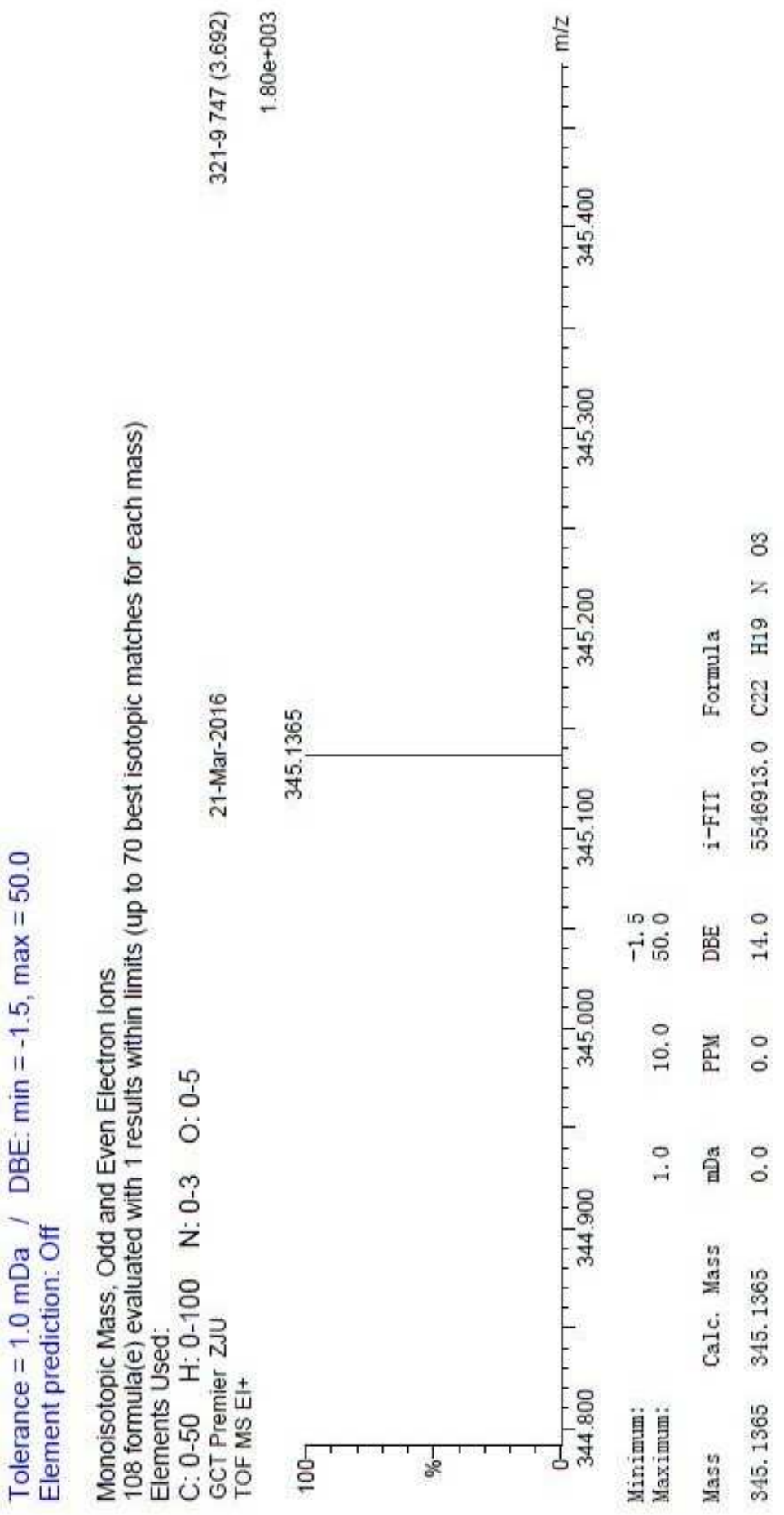


${ }^{1} \mathrm{H} \quad$ NMR Spectrum of methyl 6-oxo-6,7-dihydro-5H-dibenzo[b,d]azepine-7carboxylate 3p

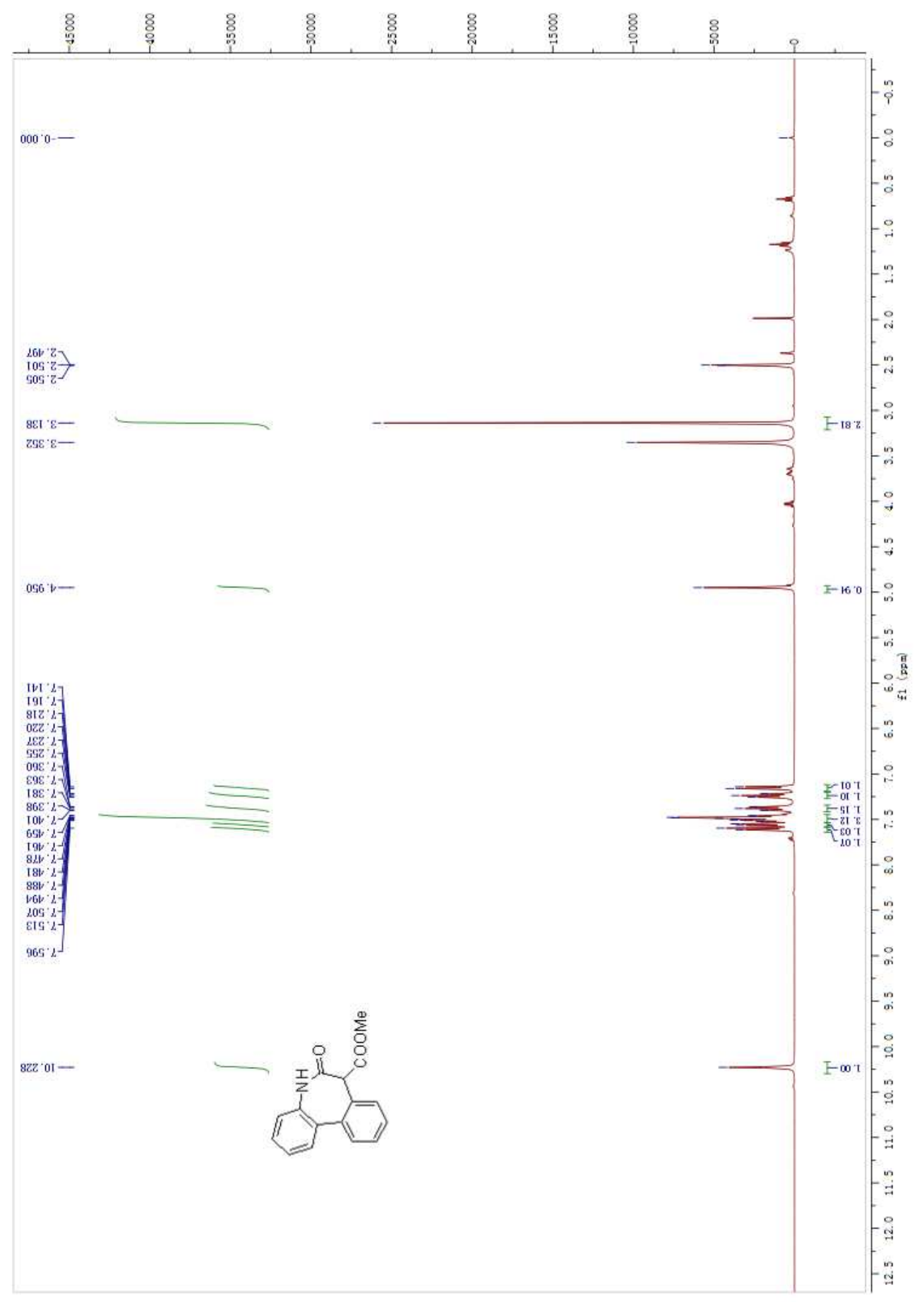


${ }^{13} \mathrm{C}$ NMR Spectrum of methyl 6-oxo-6,7-dihydro-5H-dibenzo[b,d]azepine-7-carboxy late $\mathbf{3 p}$

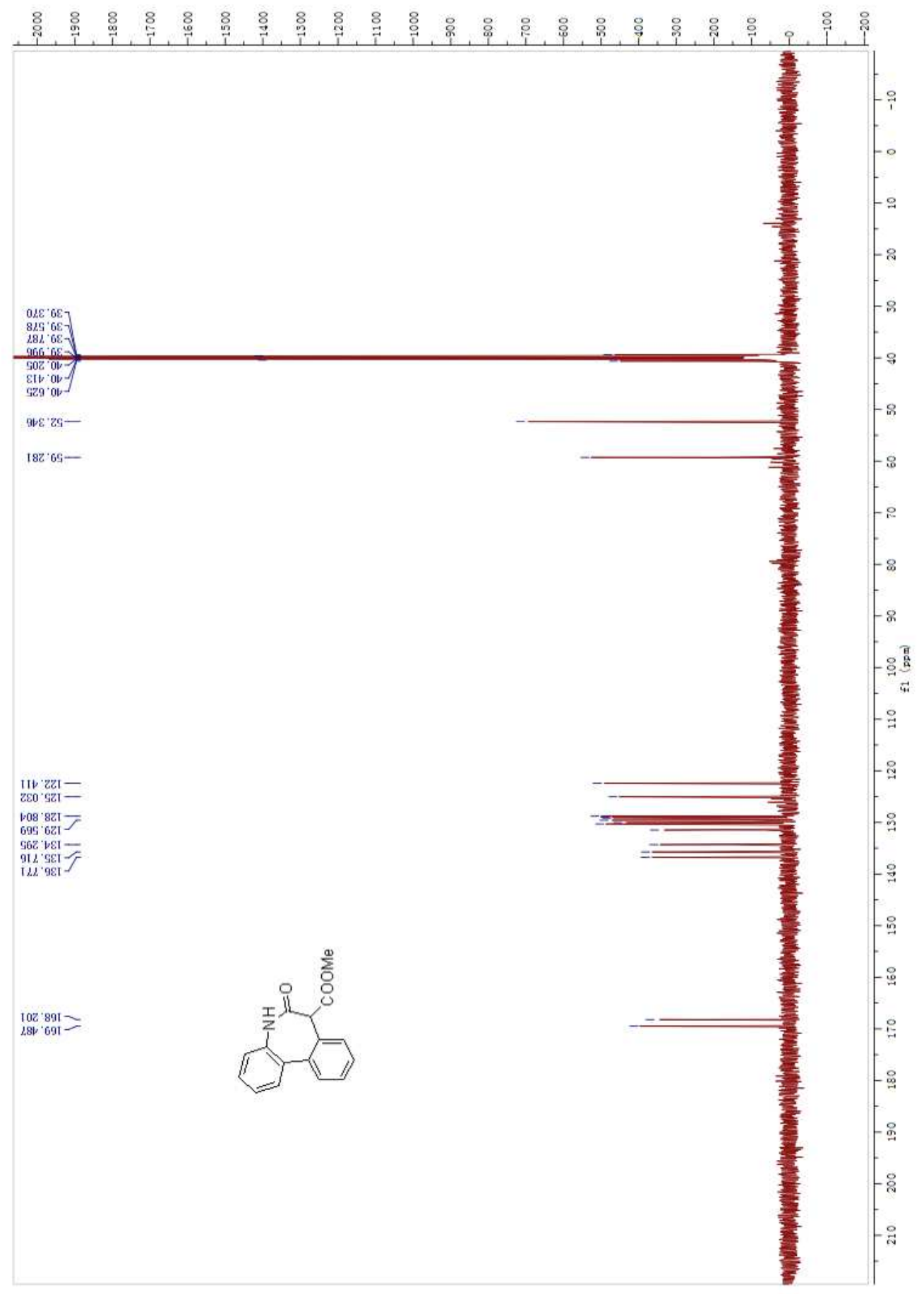


HR-MS (EI) Spectrum of methyl 6-oxo-6,7-dihydro-5H-dibenzo[b,d]azepine-7 carboxylate 3p

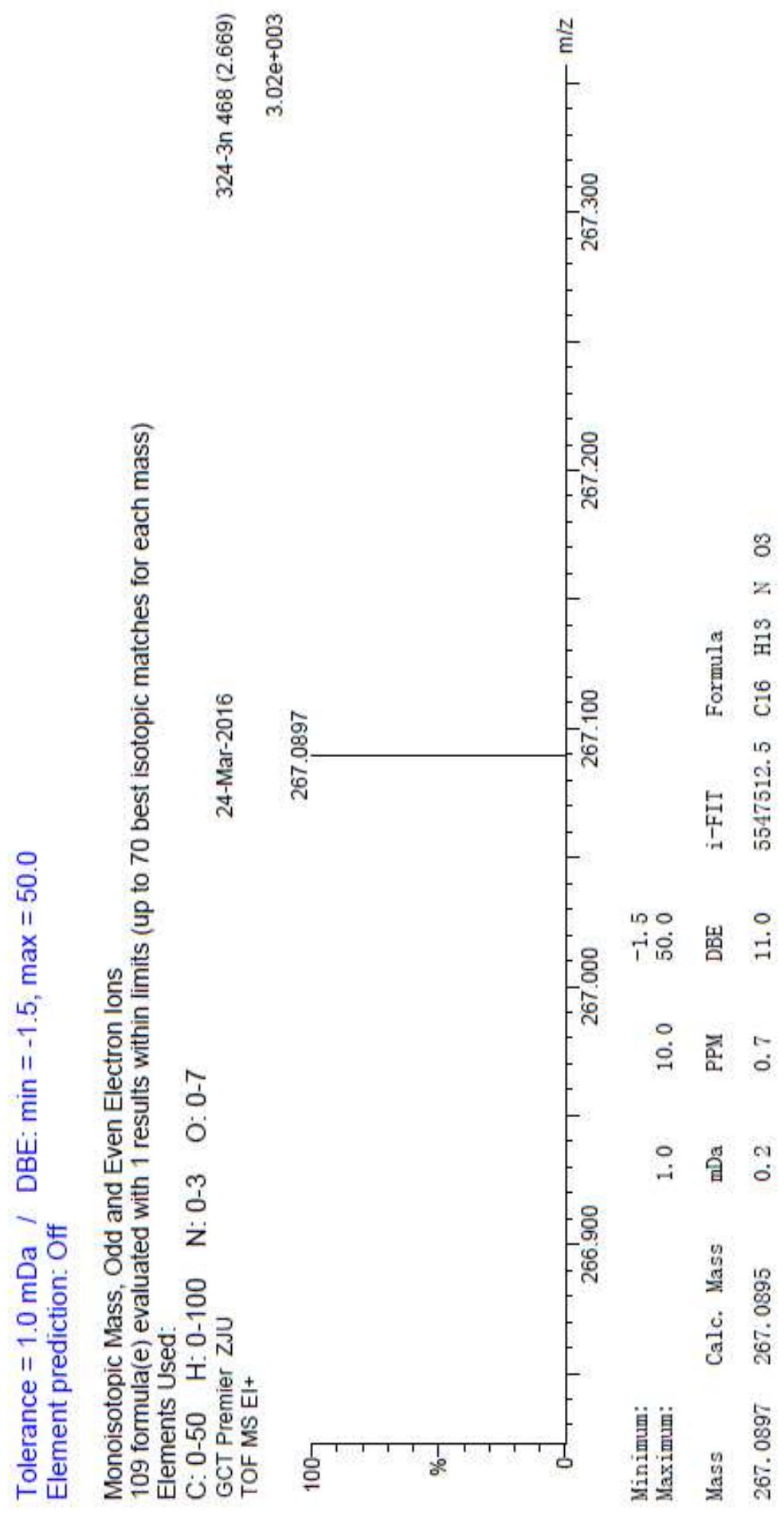


${ }^{1} \mathrm{H}$ NMR Spectrum of isopropyl 6-oxo-6,7-dihydro-5H-dibenzo[b,d]azepine-7carboxy late 3q

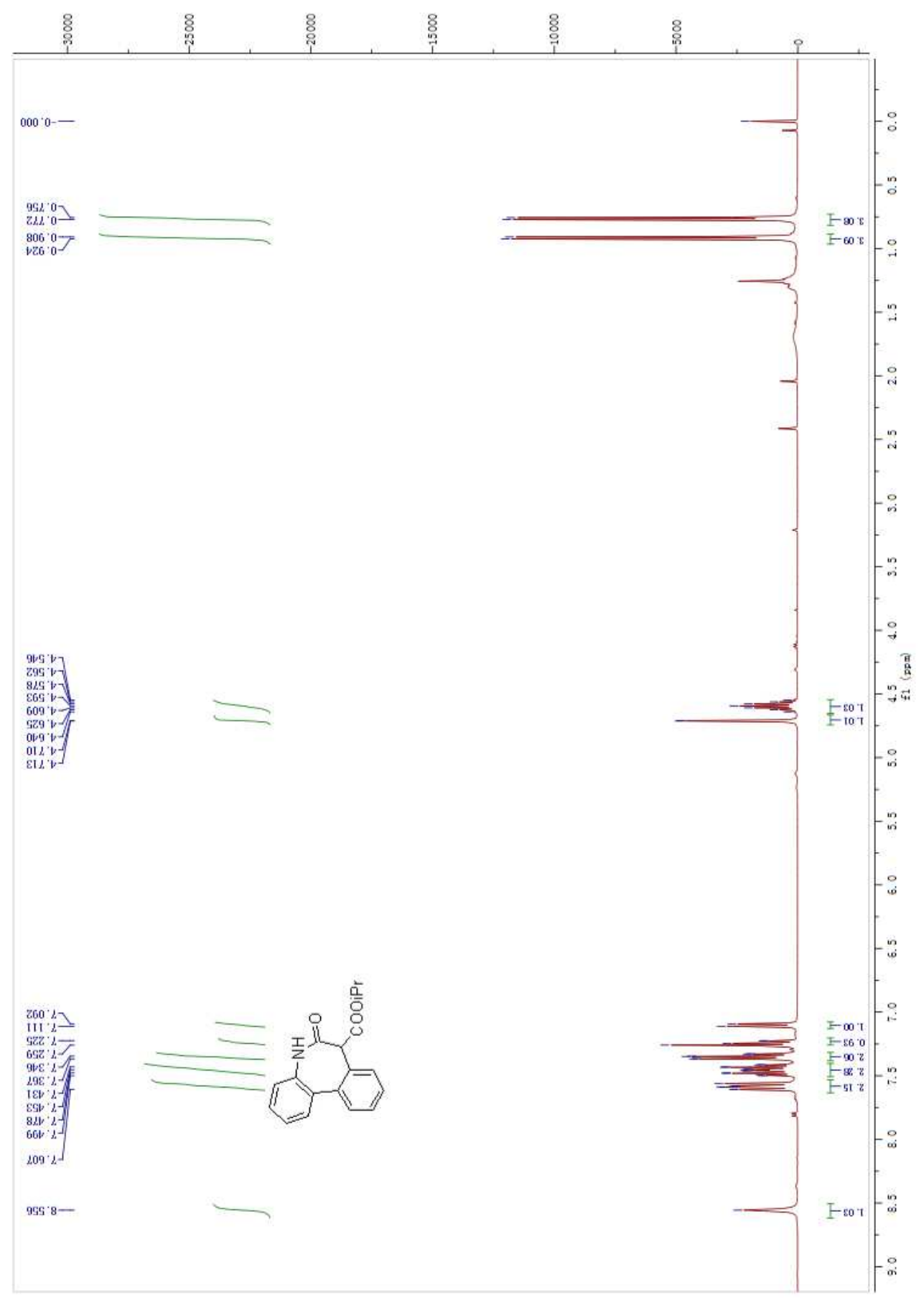


${ }^{13} \mathrm{C}$ NMR Spectrum of isopropyl 6-oxo-6,7-dihydro-5H-dibenzo[b,d]azepine-7carboxy late 3q

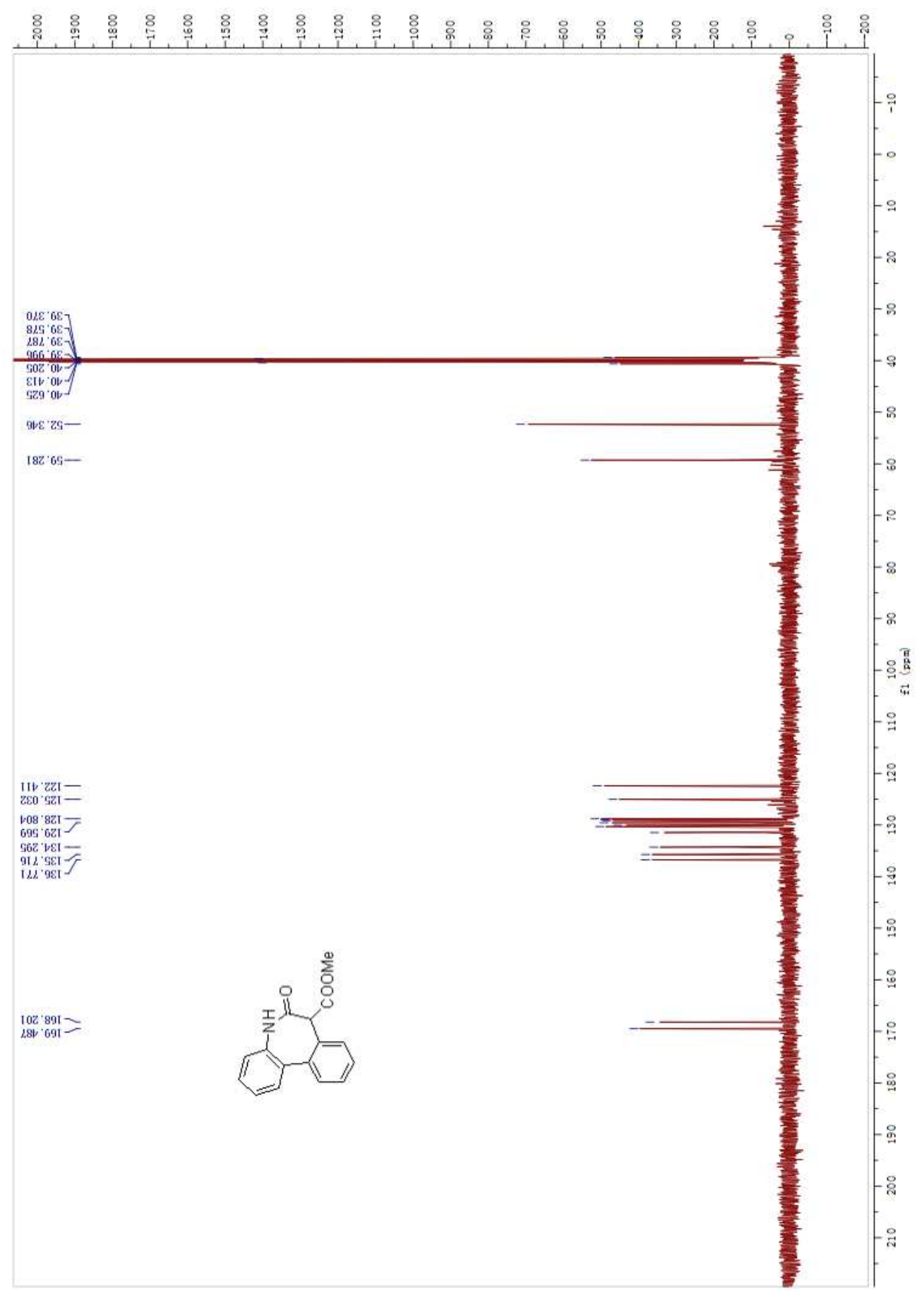


HR-MS (EI) Spectrum of isopropyl 6-oxo-6,7-dihydro-5H-dibenzo[b,d]azepine -7carboxy late $\mathbf{3 q}$

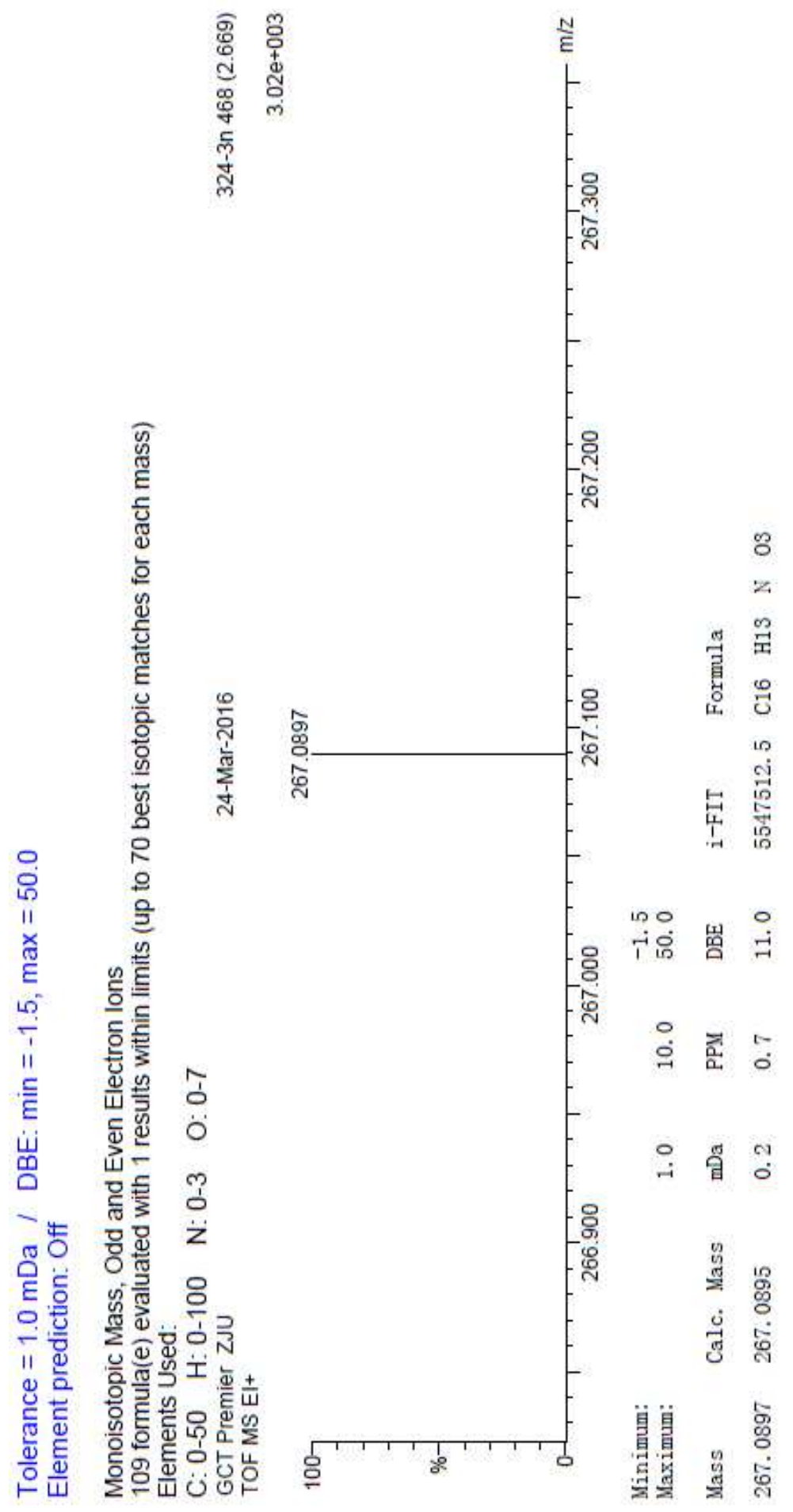


${ }^{1} \mathrm{H}$ NMR Spectrum of benzyl 9-chloro-6-oxo-6,7-dihydro-5H-dibenzo[b,d]azepine-7carboxy late $3 r$

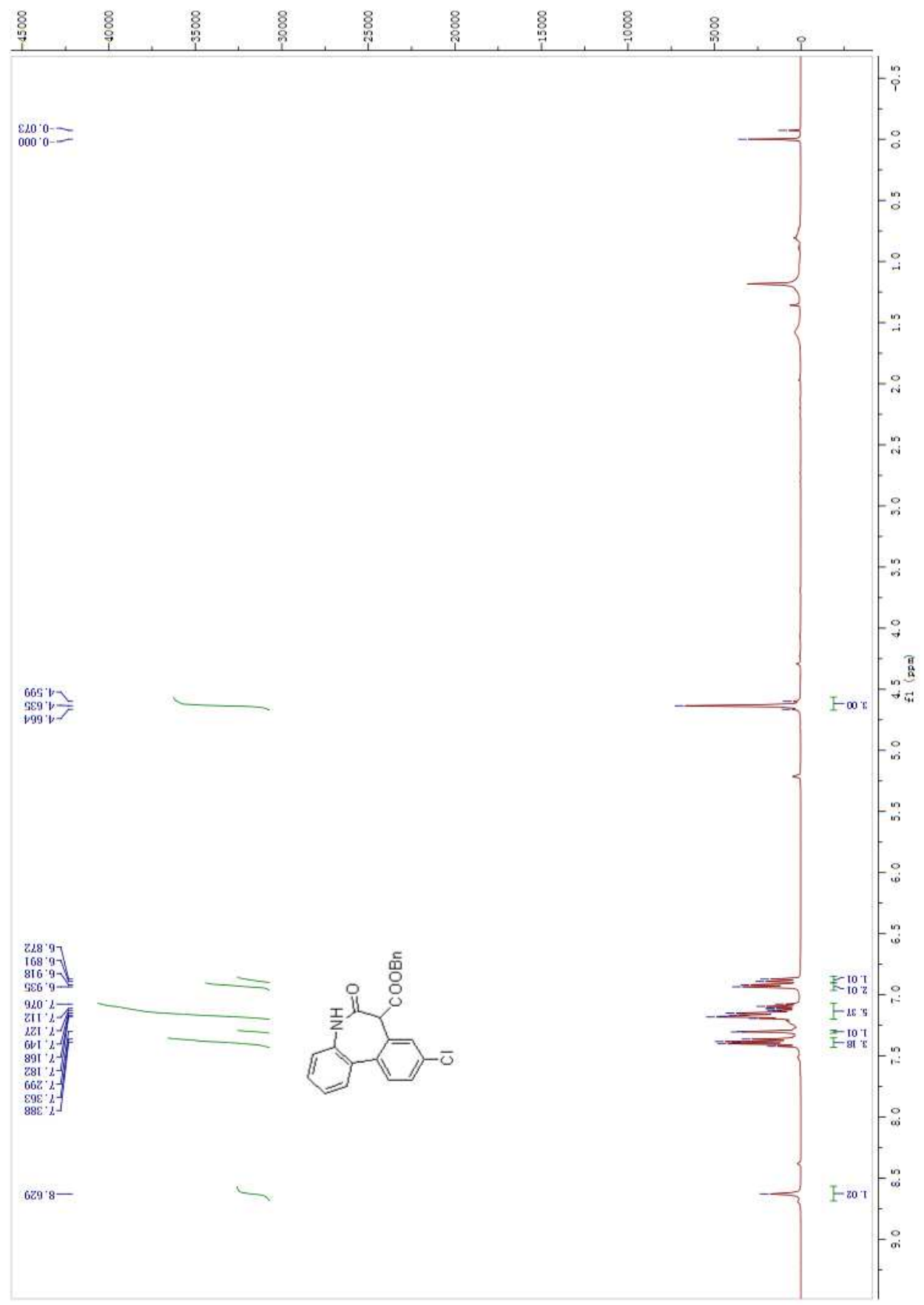


${ }^{13}$ C NMR Spectrum of benzyl 9-chloro-6-oxo-6,7-dihydro-5H-dibenzo[b,d]azepine-7 -carboxylate $\mathbf{3 r}$

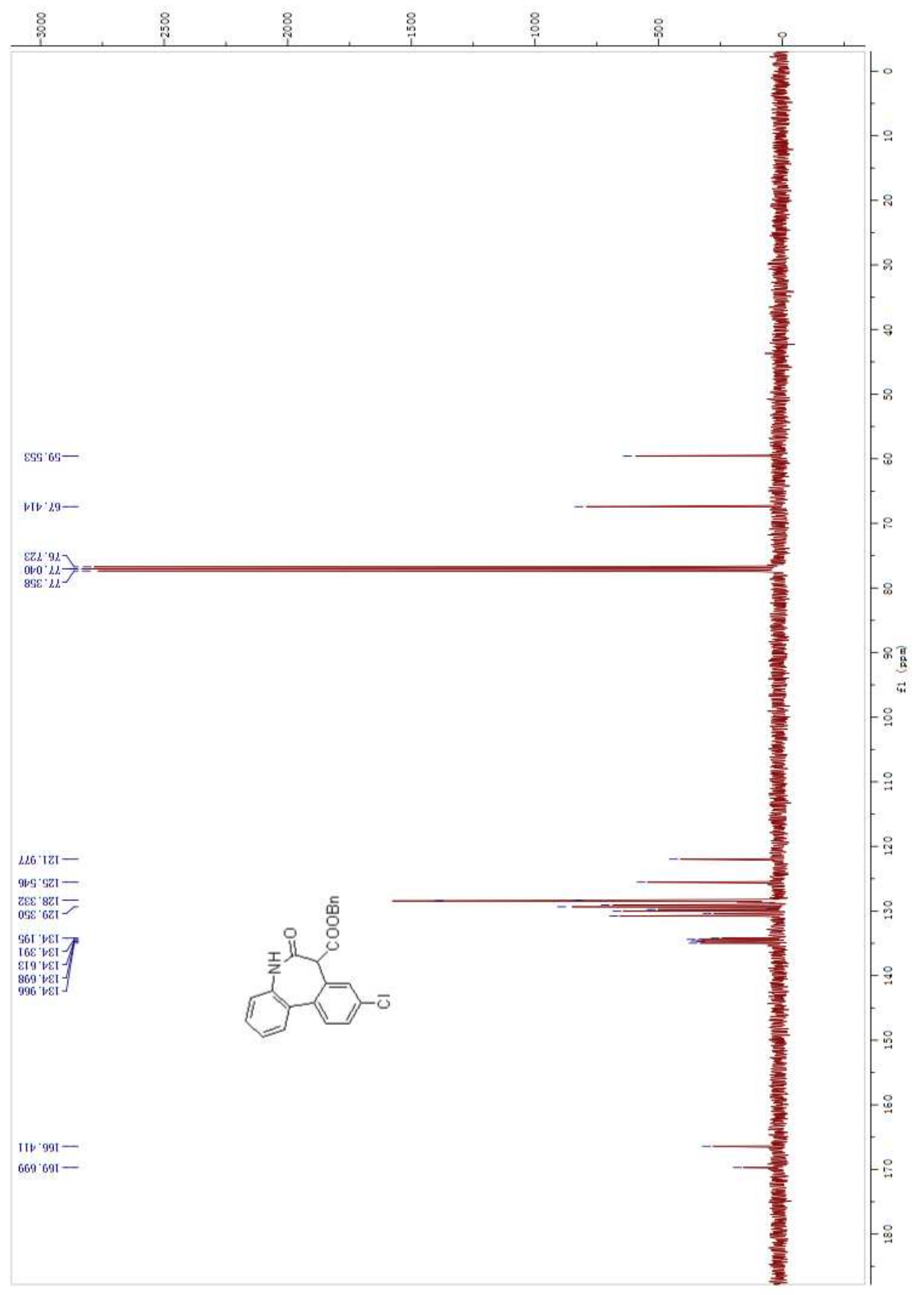


HR-MS Spectrum (EI) of benzyl 9-chloro-6-oxo-6,7-dihydro-5H-dibenzo[b,d]azepine -7-carboxylate 3r

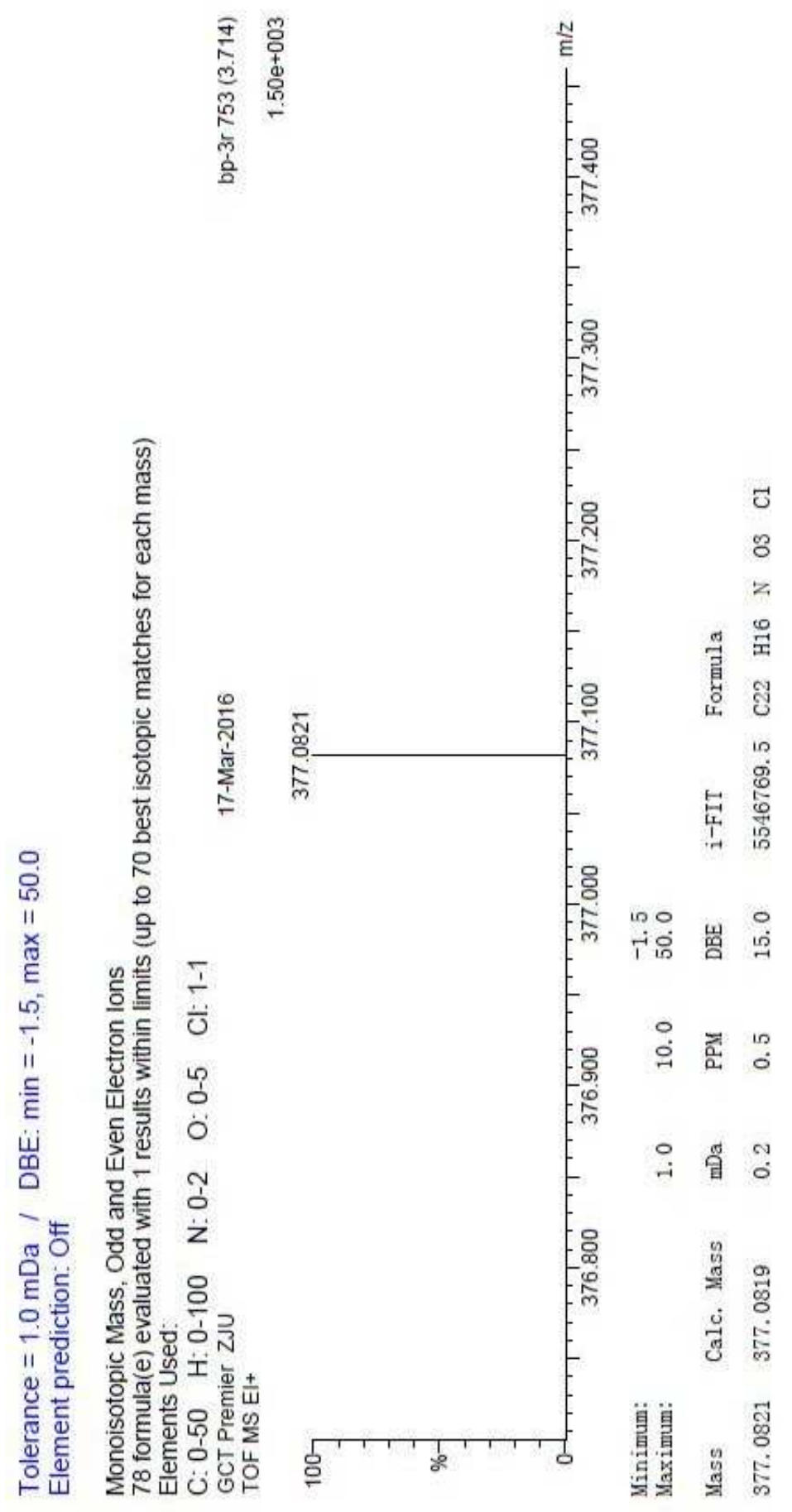


${ }^{1} \mathrm{H}$ NMR Spectrum of benzyl 6-oxo-6,7-dihydro-5H-dibenzo[b,d]azepine-7-carboxy late $3 \mathrm{~s}$

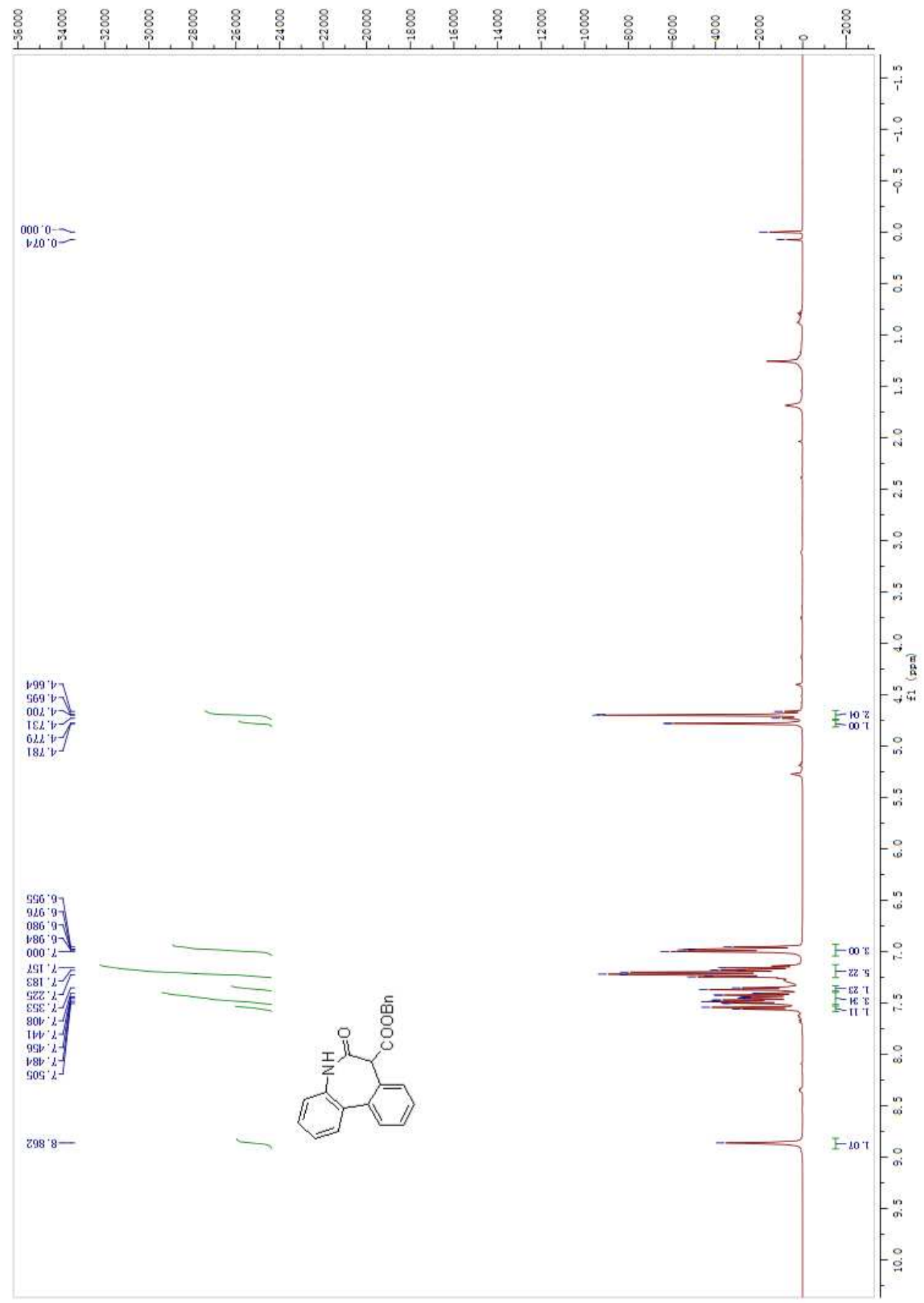


${ }^{13} \mathrm{C}$ NMR Spectrum of benzyl 6-oxo-6,7-dihydro-5H-dibenzo[b,d]azepine-7-carboxy late $3 \mathrm{~s}$

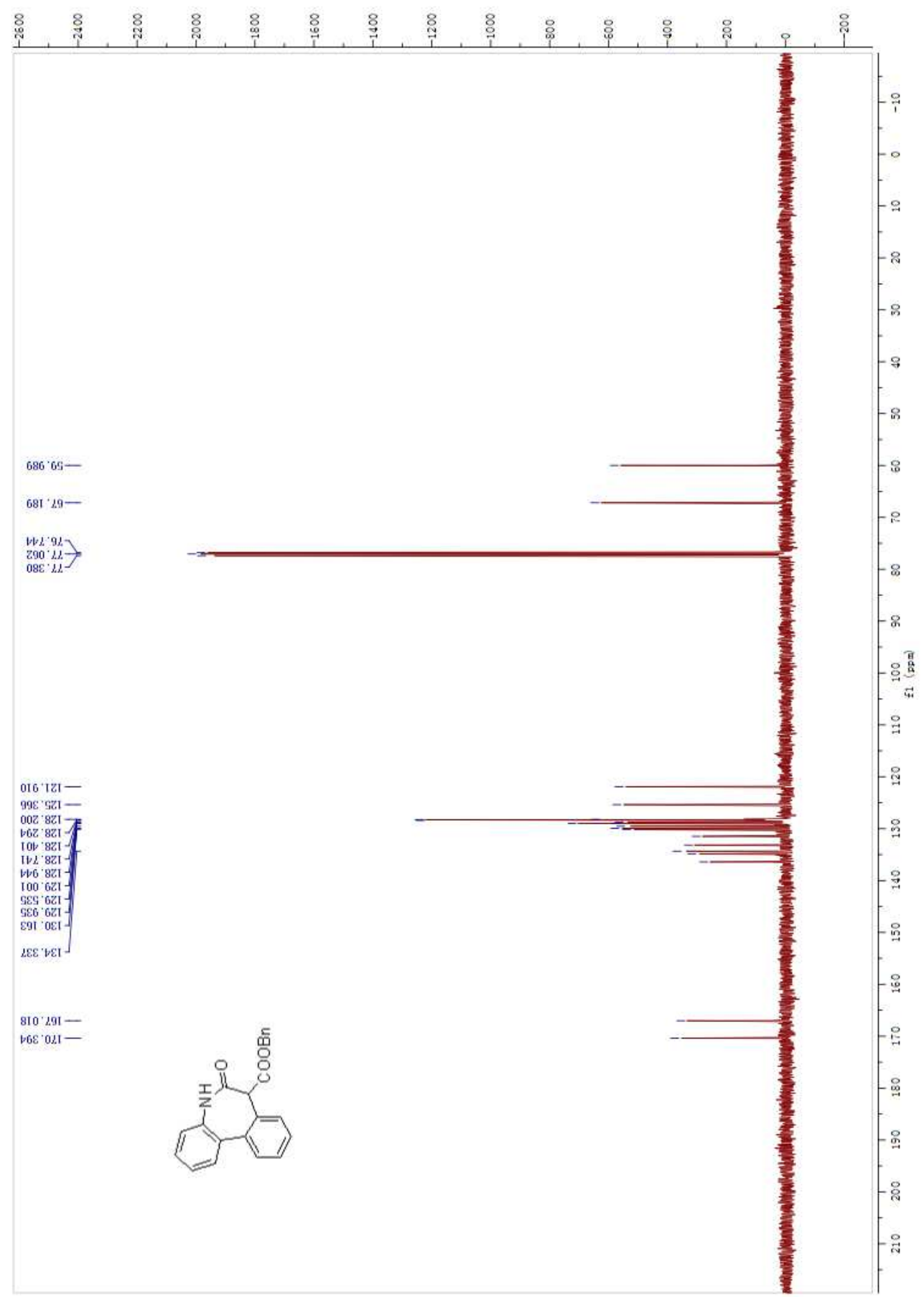


HR-MS (EI) Spectrum of benzyl 6-oxo-6,7-dihydro-5H-dibenzo[b,d]azepine-7carboxylate 3s

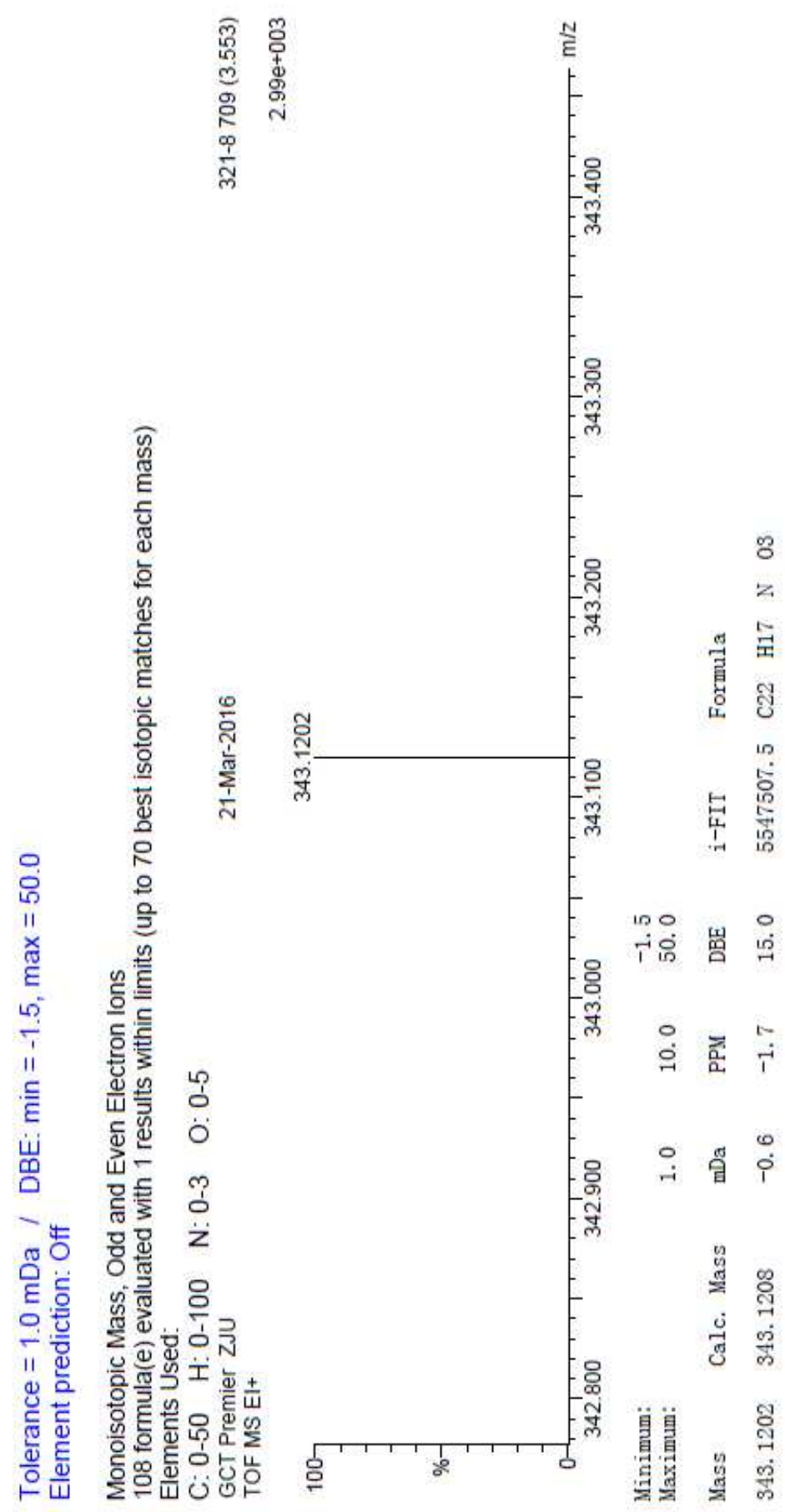


${ }^{1} \mathrm{H}$ NMR Spectrum of tert-butyl 6-oxo-6,7-dihydro-5H-dibenzo[b,d]azepine-7carboxylate $\mathbf{3 t}$

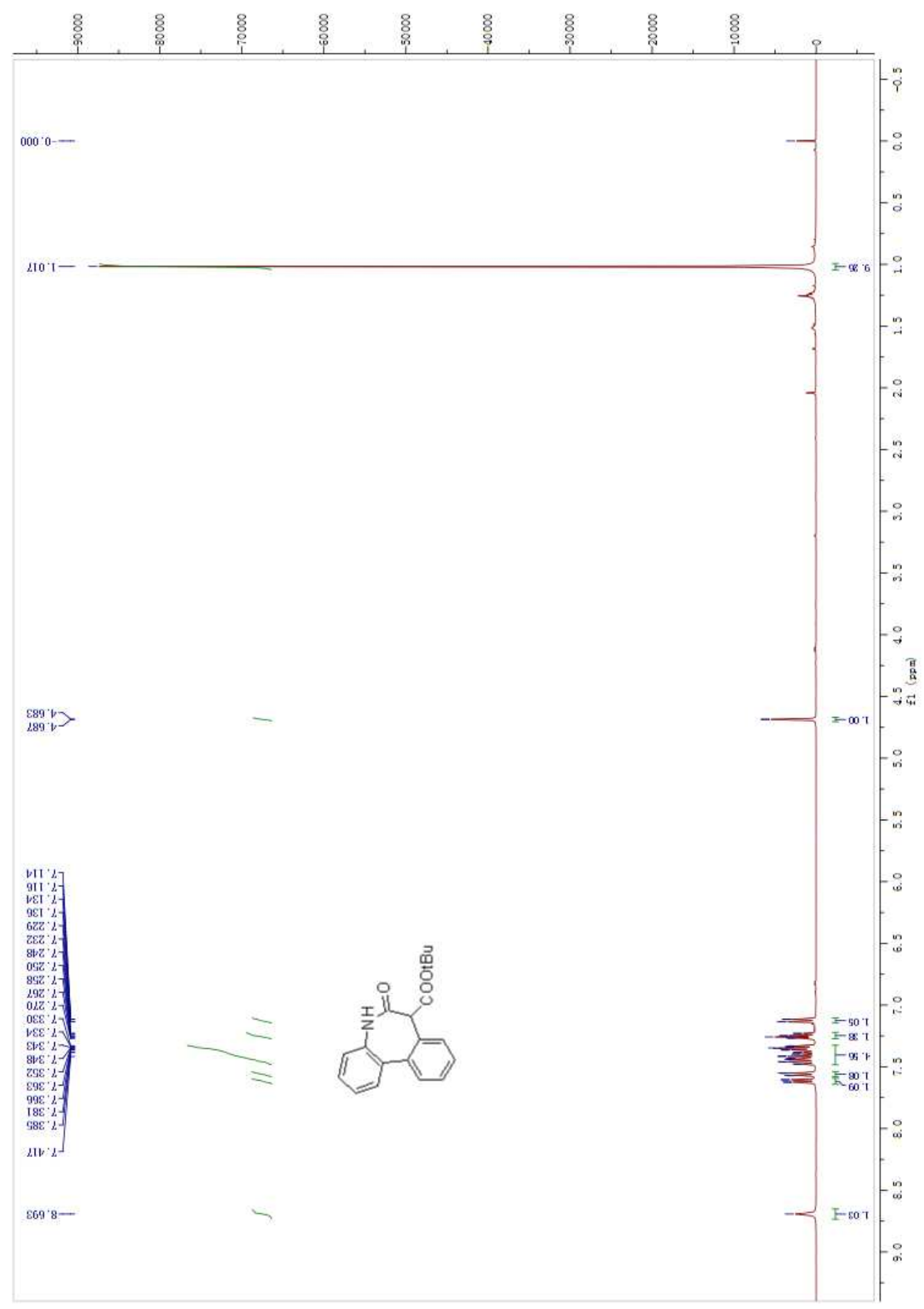


${ }^{13} \mathrm{C}$ NMR Spectrum of tert-butyl 6-oxo-6,7-dihydro-5H-dibenzo[b,d]azepine-7carboxylate 3t

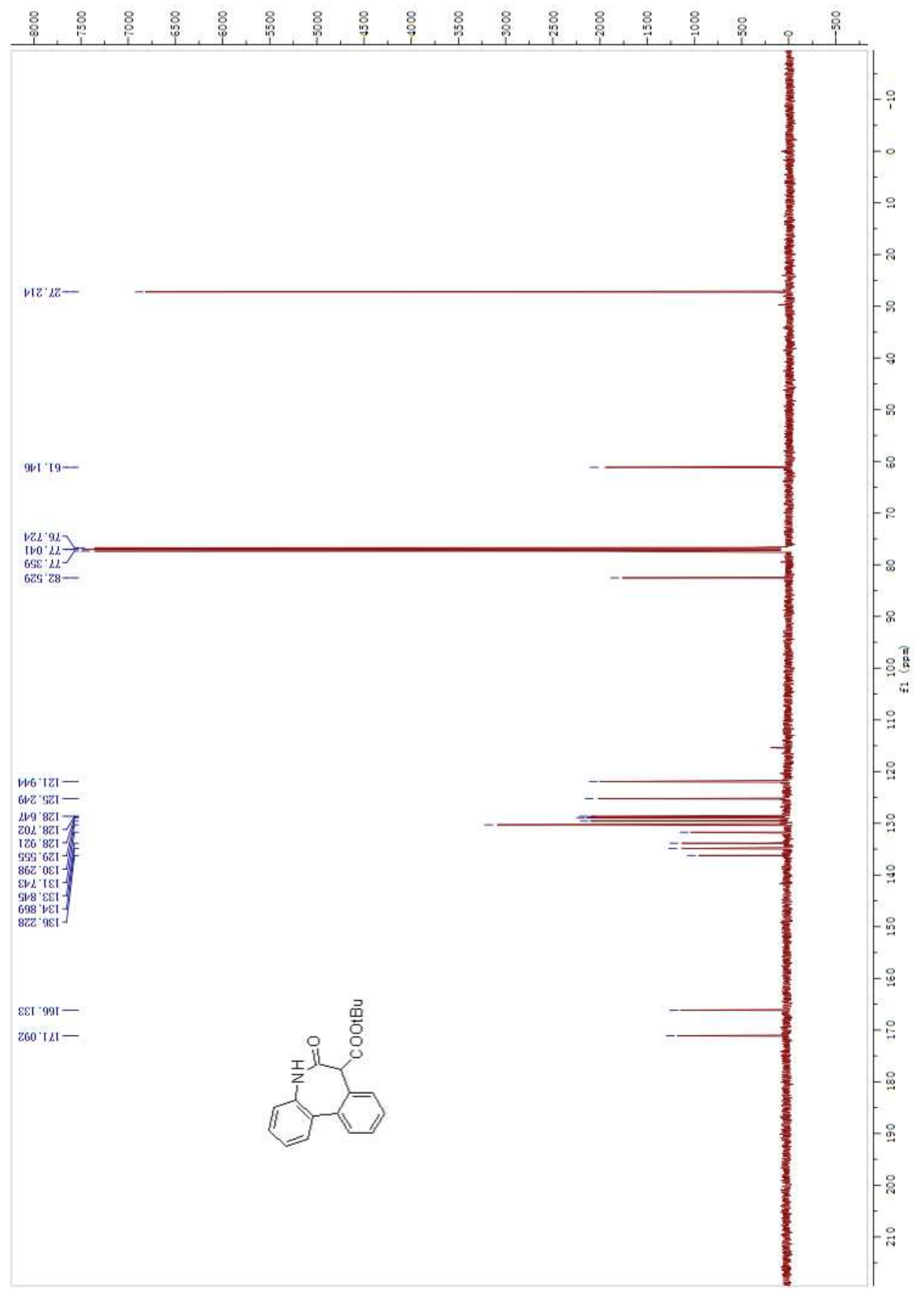


HR-MS (EI) Spectrum of tert-butyl 6-oxo-6,7-dihydro-5H-dibenzo[b,d]azepine7-carboxy late $\mathbf{3 t}$

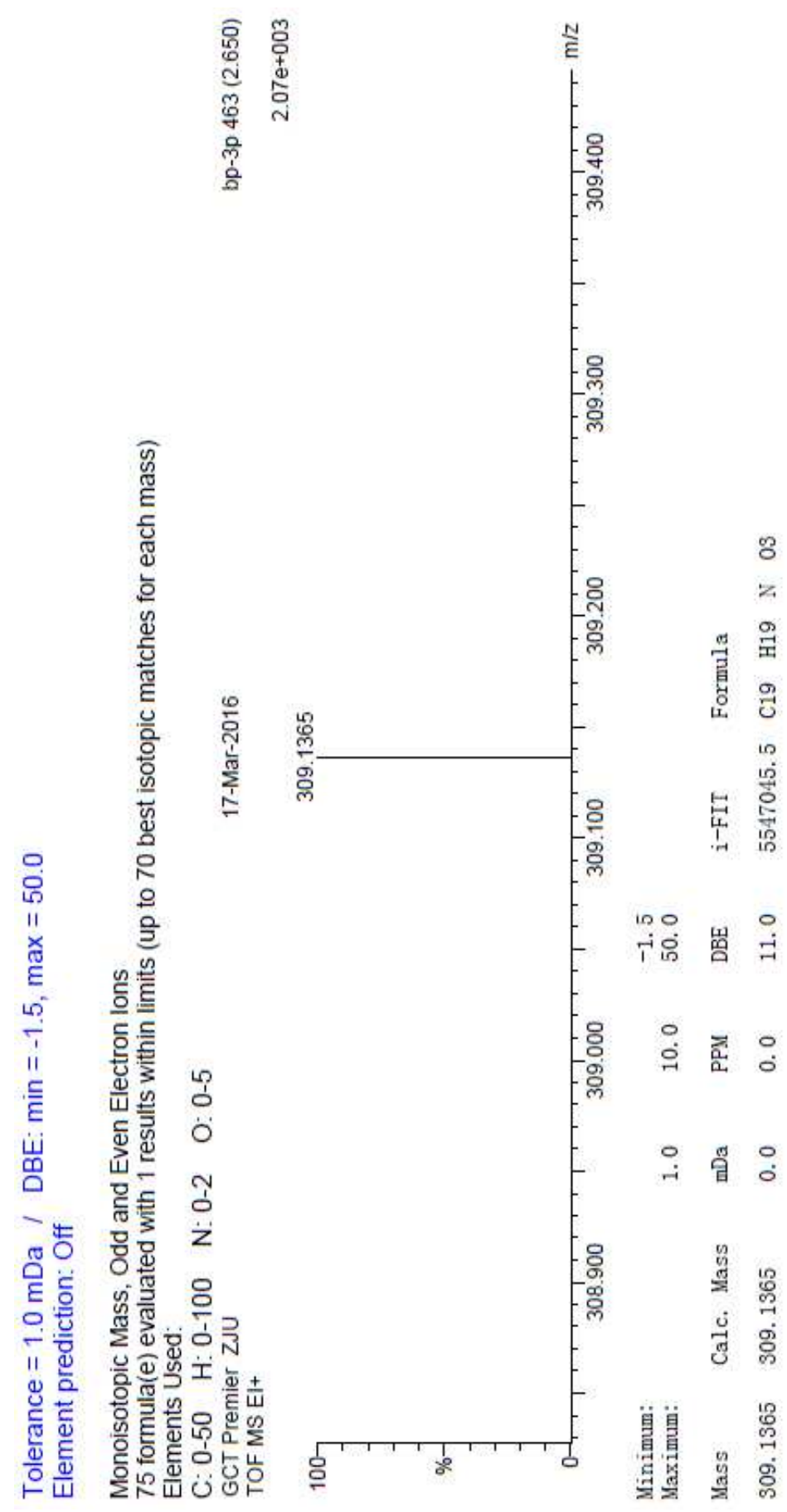


${ }^{1} \mathrm{H}$ NMR Spectrum of ethyl 5-oxo-5,6-dihydro-4H-benzo[b]furo[3,2-d]azepine-4carboxylate 3u

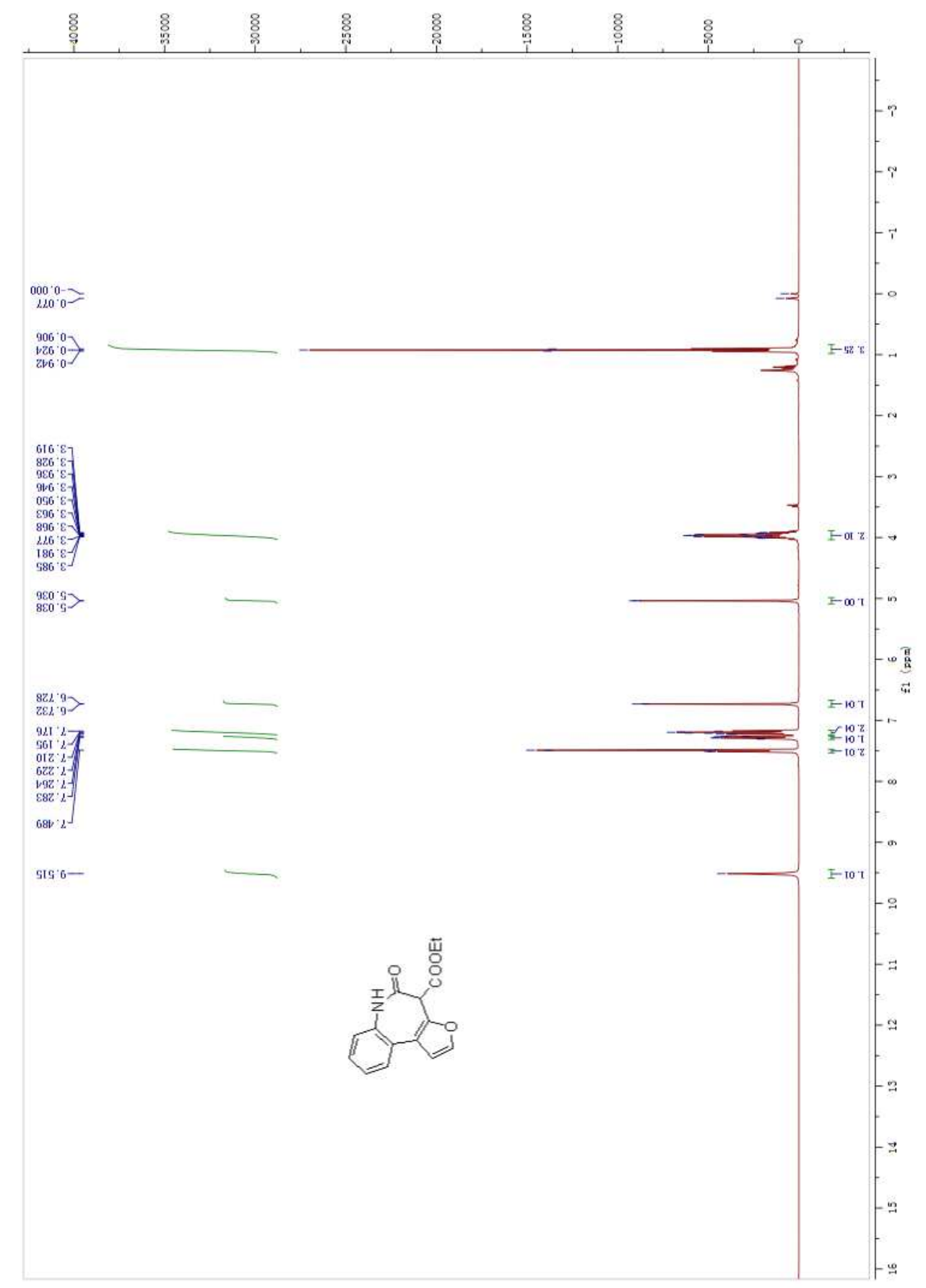


${ }^{13} \mathrm{C}$ NMR Spectrum of ethyl 5-oxo-5,6-dihydro-4H-benzo[b]furo[3,2-d]azepine-4carboxylate $\mathbf{3 u}$

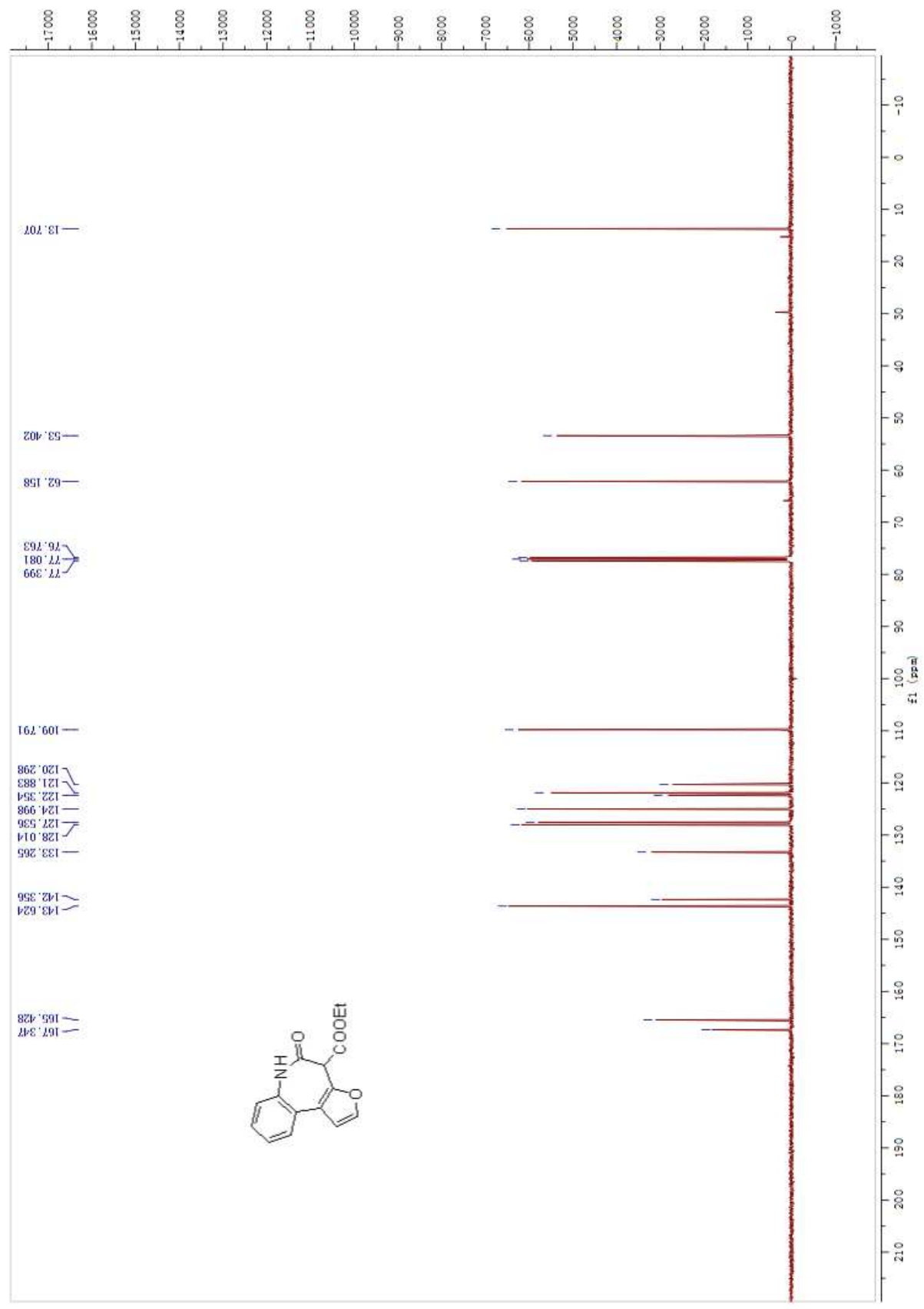


HR-MS (EI) Spectrum of ethyl 5-oxo-5,6-dihydro-4H-benzo[b]furo[3,2-d]azepine-4carboxylate $3 \mathbf{u}$

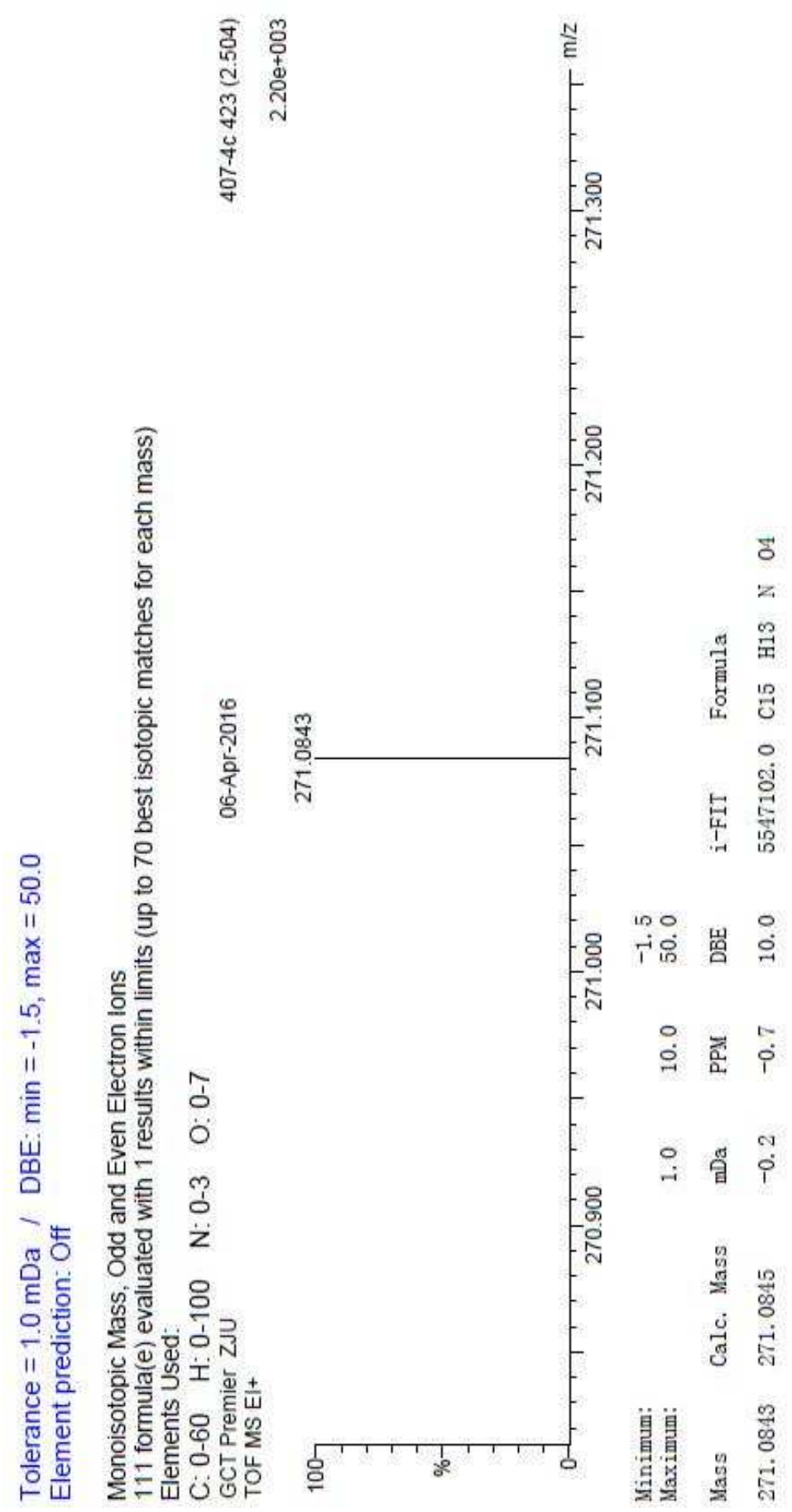


${ }^{1} \mathrm{H}$ NMR Spectrum of ethyl 5-oxo-5,6-dihydro-4H-benzo[b]thieno[3,2-d]azepine-4carboxylate $3 \mathbf{v}$

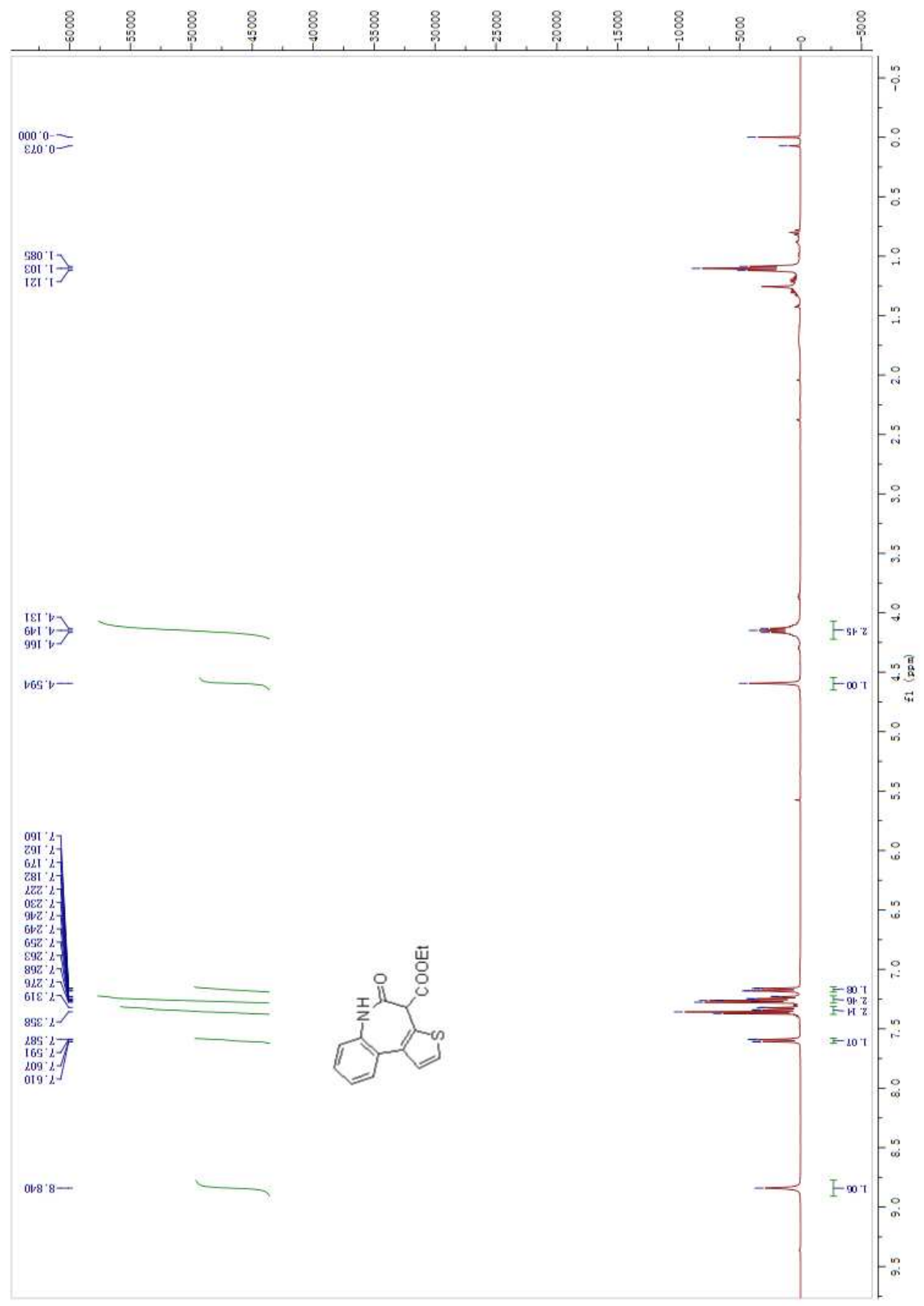


${ }^{13} \mathrm{C}$ NMR Spectrum of ethyl 5-oxo-5,6-dihydro-4H-benzo[b]thieno[3,2-d]azepine-4carboxylate $3 \mathbf{v}$

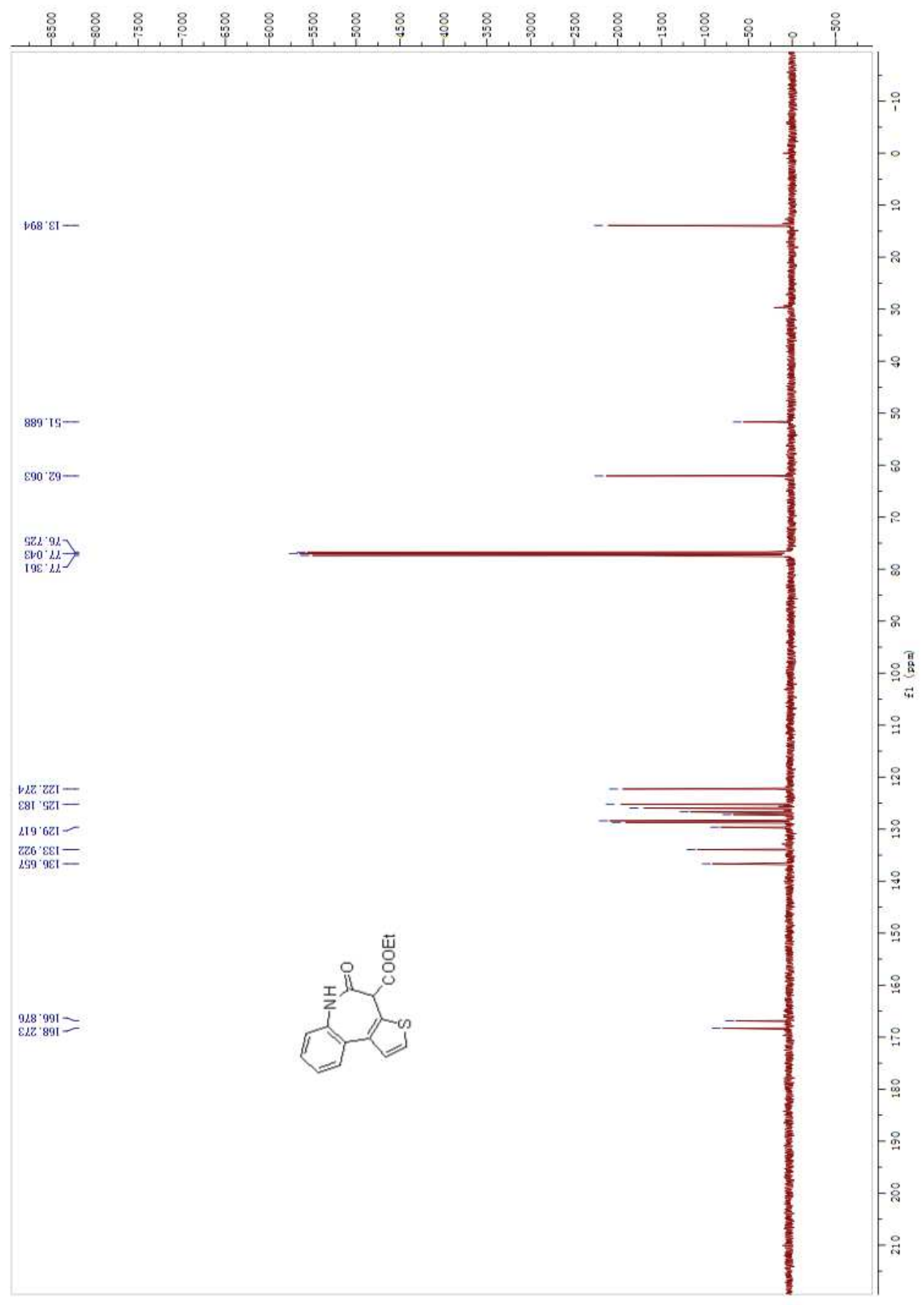


HR-MS (EI) Spectrum of ethyl 5-oxo-5,6-dihydro-4H-benzo[b] thieno[3,2-d]azepine4 - carboxylate $\mathbf{3 v}$

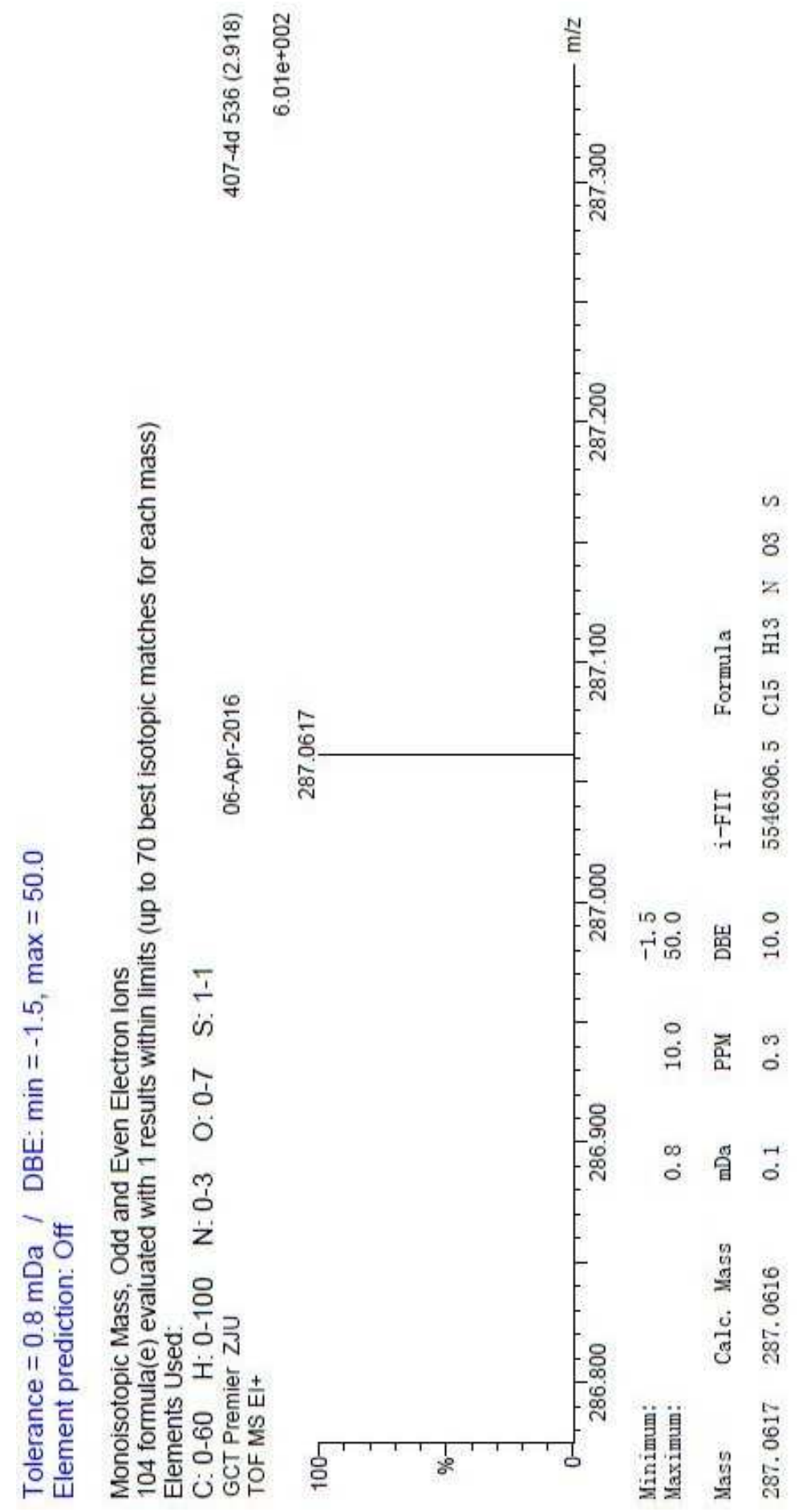


${ }^{1}$ H NMR Spectrum of ethyl 3-([1,1'-biphenyl]-2-ylamino)-2-diazo-3-oxopropanoate 4

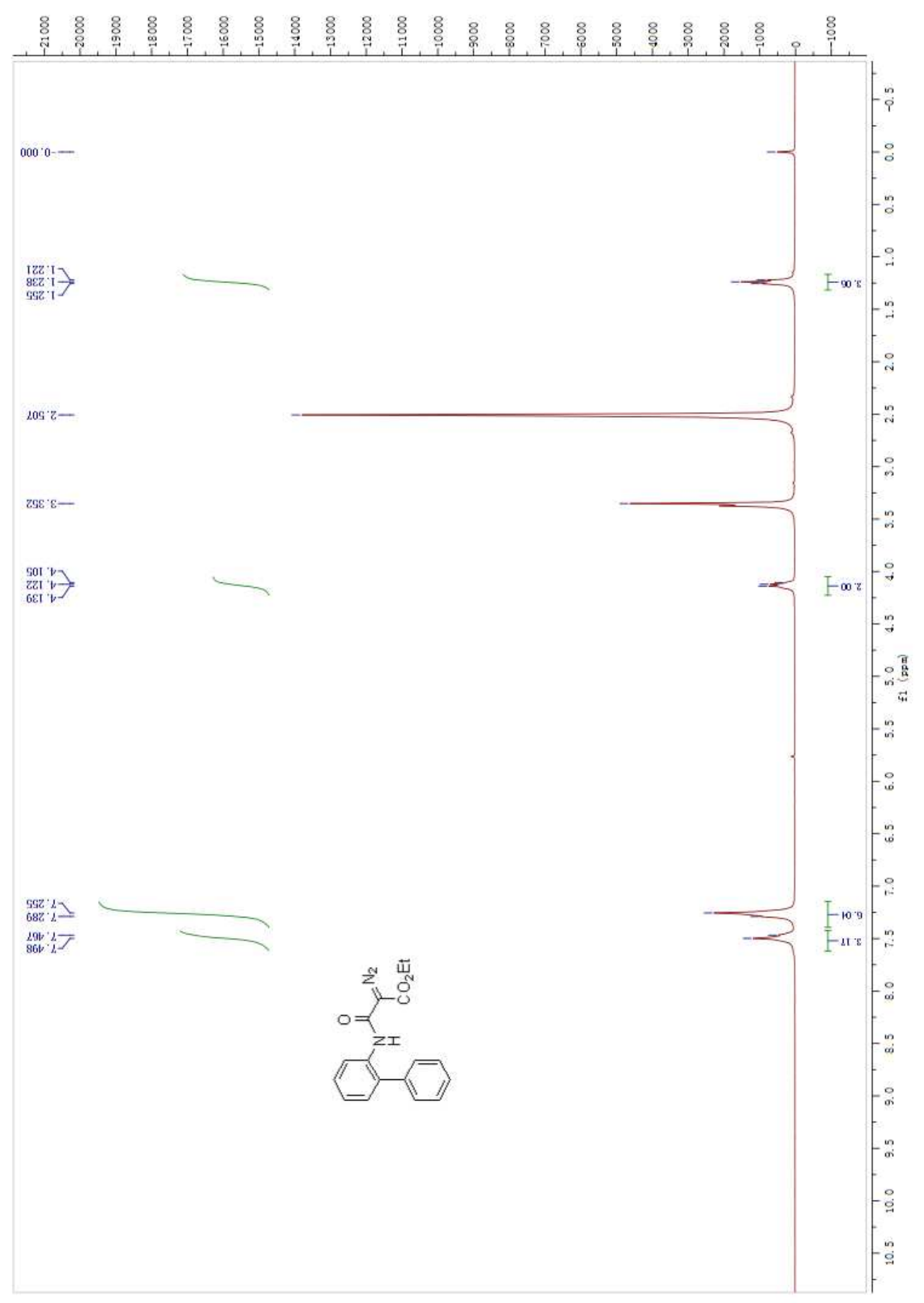


${ }^{13}$ C NMR Spectrum of ethyl 3-([1,1'-biphenyl]-2-ylamino)-2-diazo-3-oxopropanoate 4

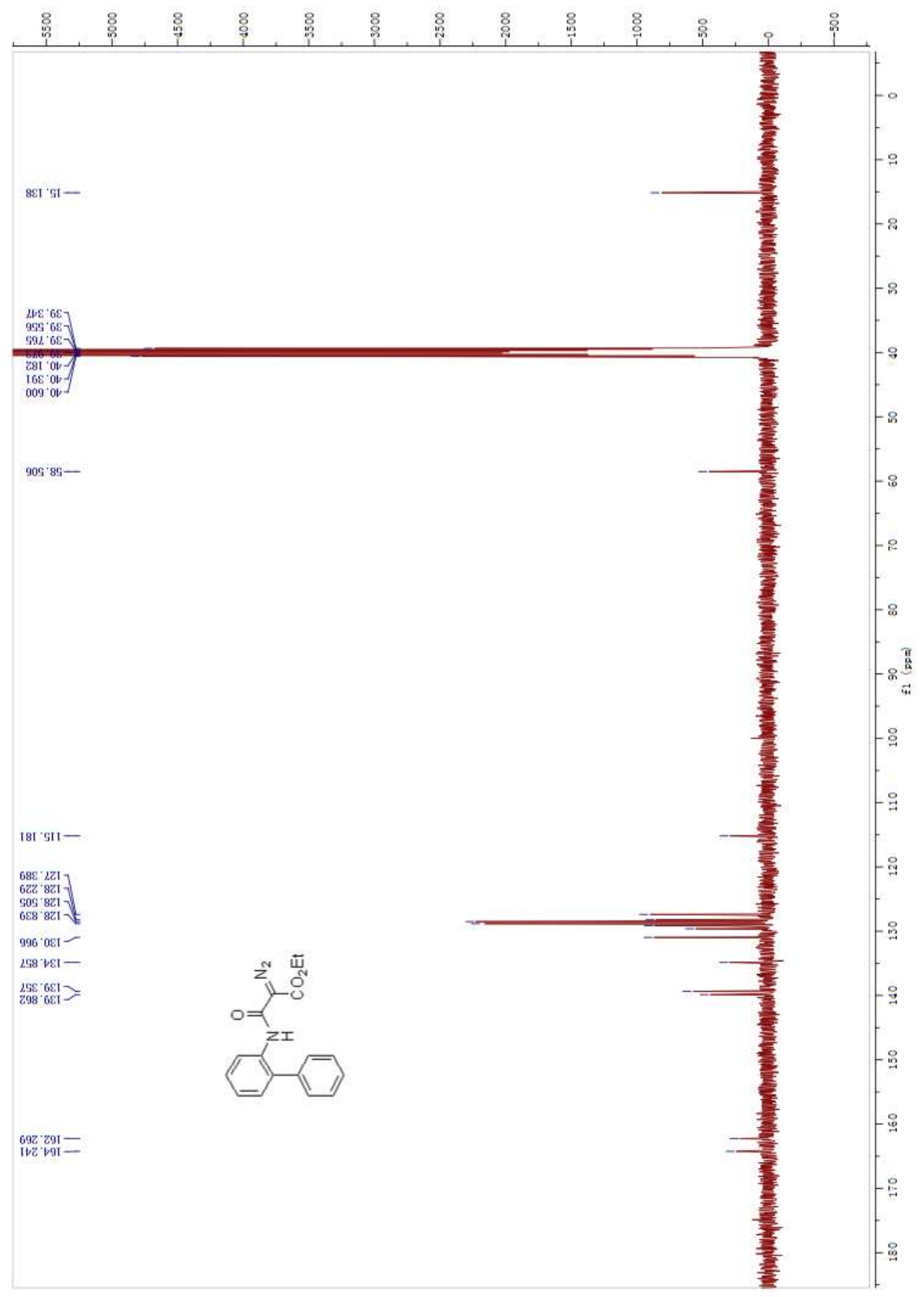


HR-MS (ESI) Spectrum of ethyl 3-([1,1'-biphenyl]-2-ylamino)-2-diazo-3-oxopropanoate 4

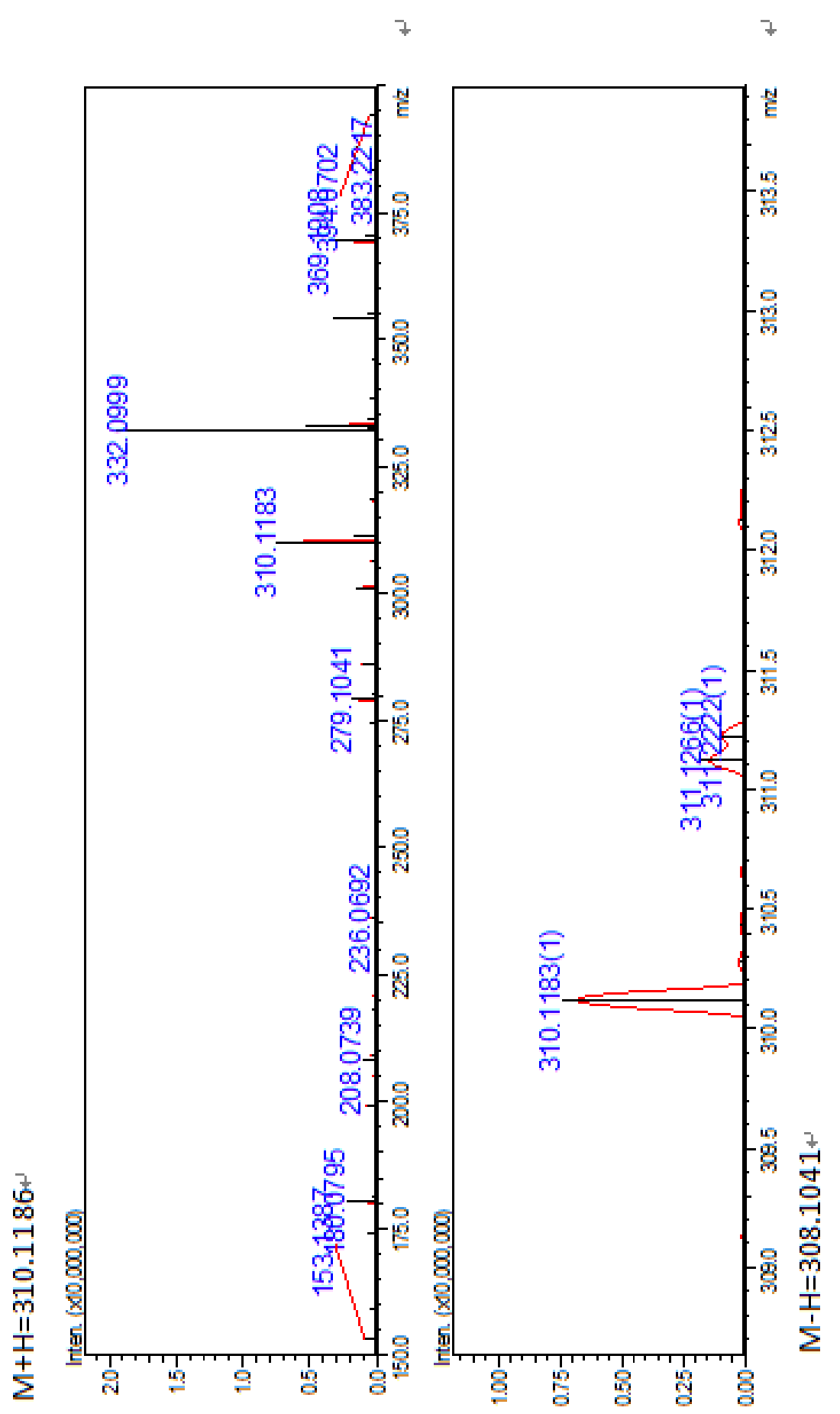


${ }^{1} \mathrm{H}$ NMR Spectrum of [1,1'-biphenyl]-2',3',4',5',6'-d5-2-amine $\mathbf{1 a - D _ { 5 }}$

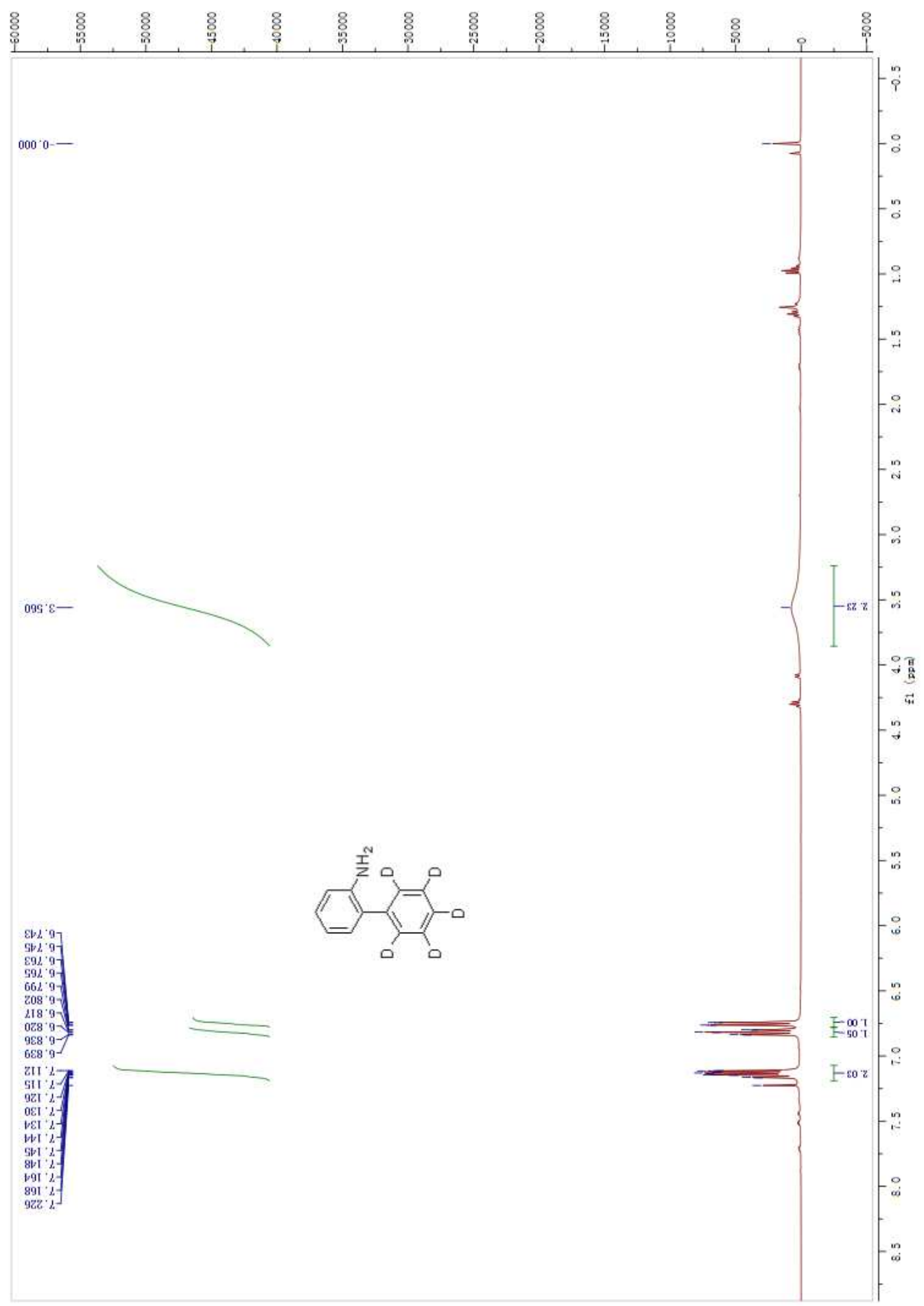


${ }^{13} \mathrm{C}$ NMR Spectrum of [1, 1'-biphenyl]-2',3',4',5',6'-d5-2-amine 1a-D

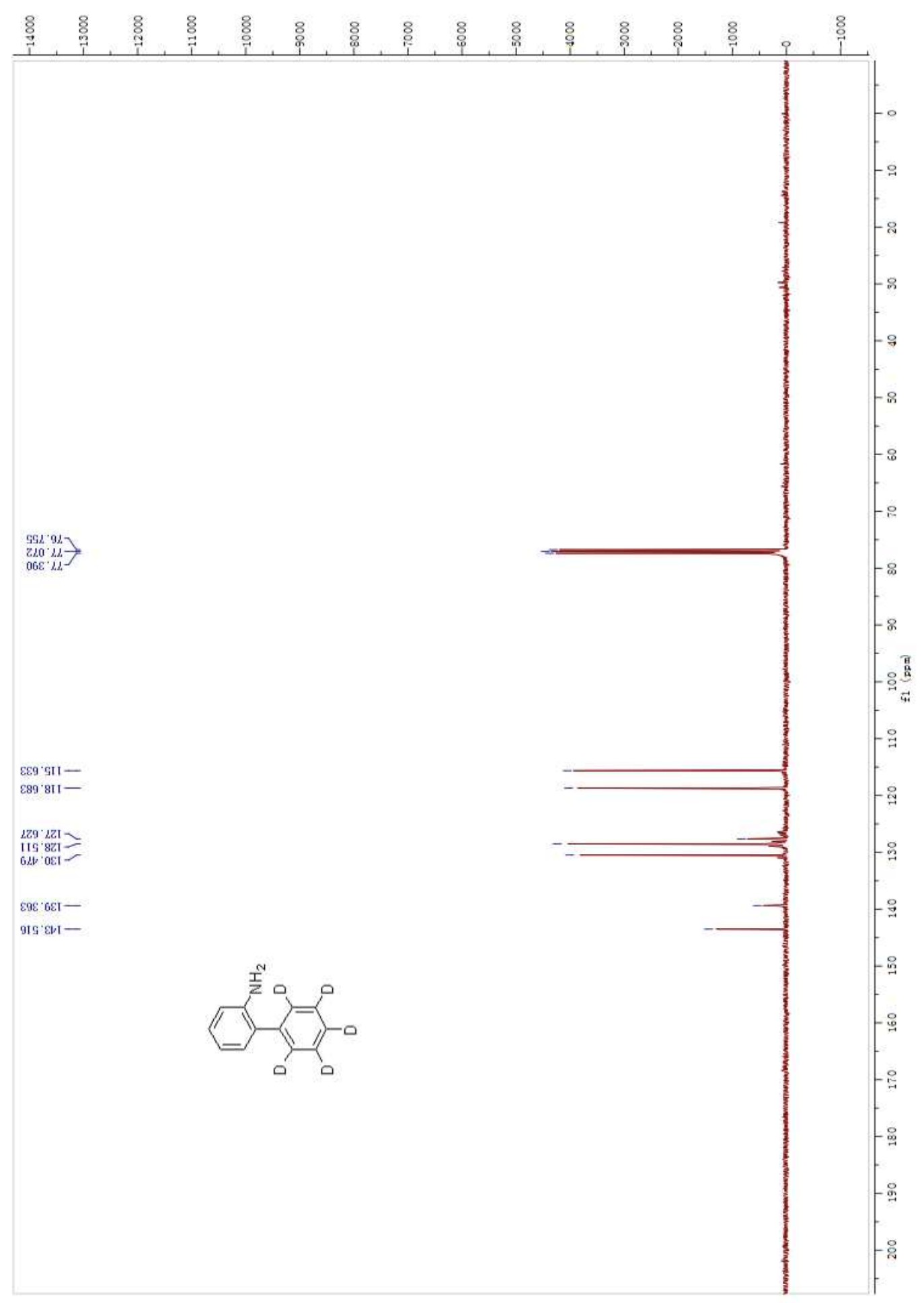


HR-MS (EI) Spectrum of [1,1'-biphenyl]-2',3',4',5',6'-d5-2-amine 1a-D

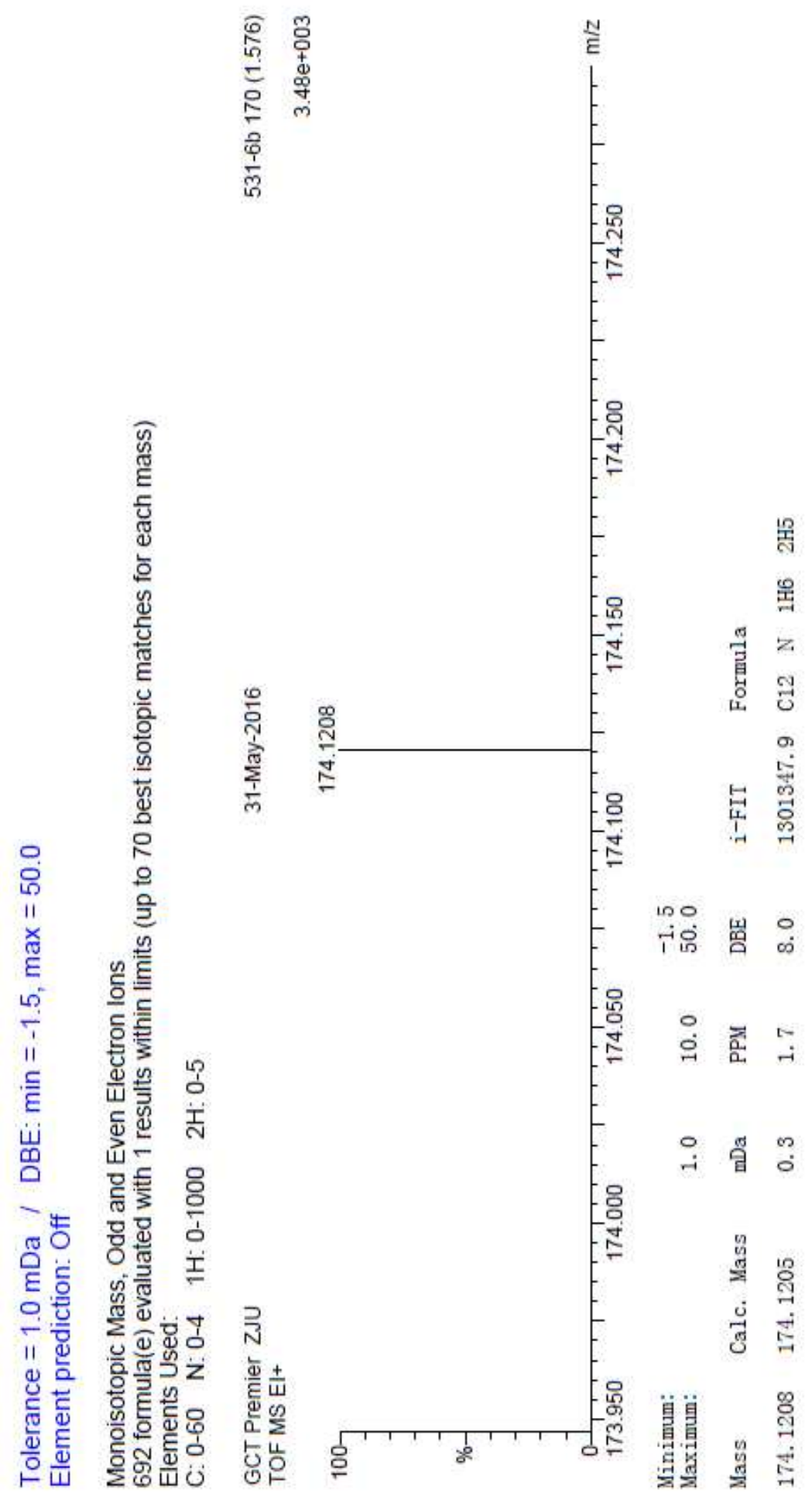


${ }^{1} \mathrm{H}$ NMR Spectrum of The Mixture of Derivatives 3a and Deuterated Azepinone Derivatives 3a-D 4

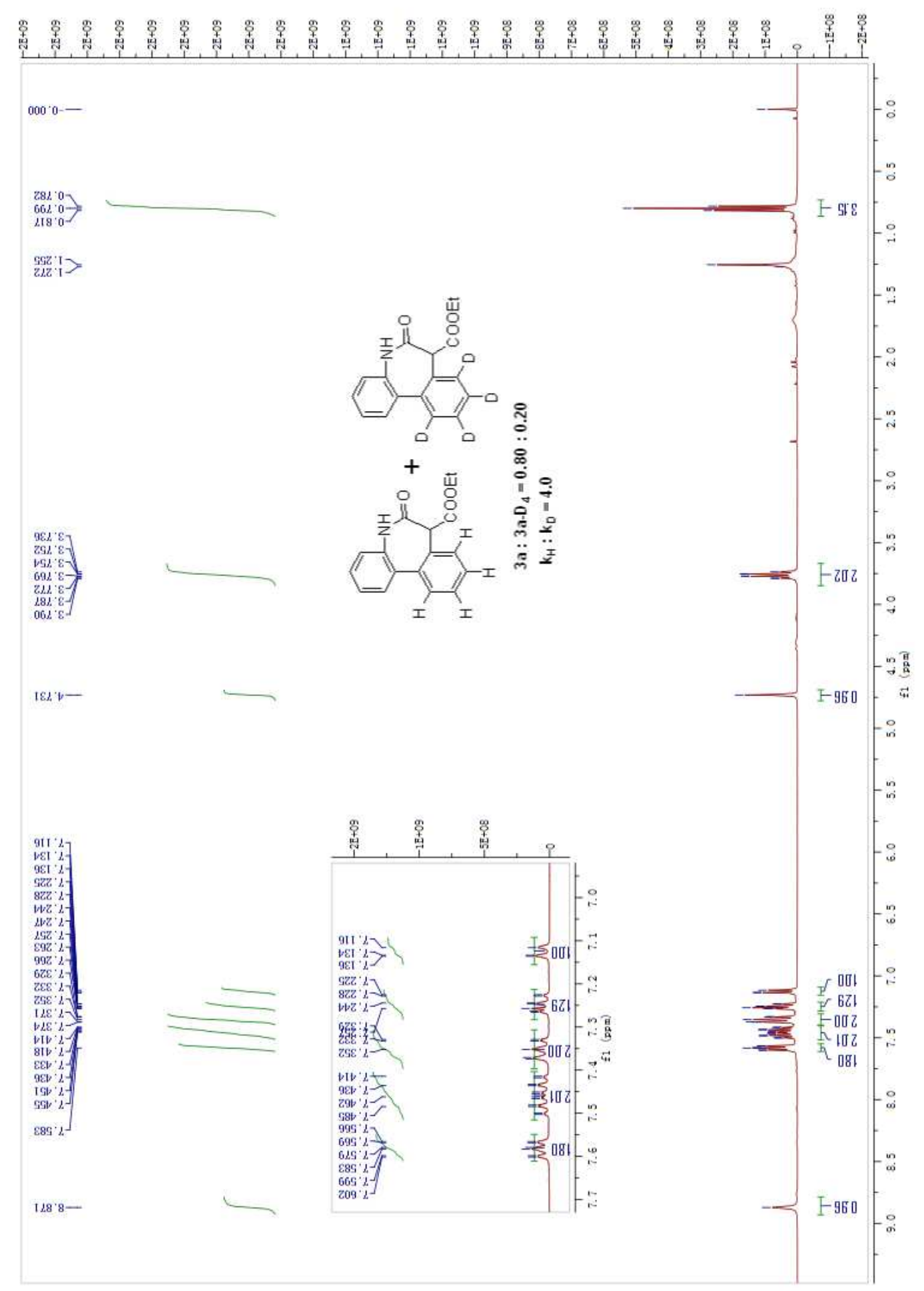

